\title{
Loop Corrections in the Constituent Quark Model
}

\author{
by
}

Rouzbeh Modarresi-Yazdi, B.Sc.

\author{
A thesis submitted to the \\ Faculty of Graduate and Postdoctoral Affairs \\ in partial fulfillment of the requirements for the degree of
}

Master of Science in Physics

Ottawa-Carleton Institute for Physics

Department of Physics

Carleton University

Ottawa, Ontario

August 29, 2017

(C) Copyright

Rouzbeh Modarresi-Yazdi, 2017 
The undersigned hereby recommends to the

Faculty of Graduate and Postdoctoral Affairs acceptance of the thesis

\title{
Loop Corrections in the Constituent Quark Model
}

\author{
submitted by Rouzbeh Modarresi-Yazdi, B.Sc.
}

in partial fulfillment of the requirements for the degree of

\author{
Master of Science in Physics
}

Professor Stephen Godfrey, Thesis Supervisor

Professor Alain Bellerive, Chair,

Department of Physics

Ottawa-Carleton Institute for Physics

Department of Physics

Carleton University

August, 2017 


\begin{abstract}
Meson spectroscopy has had an extremely busy and productive decade since the discovery of the $D_{s}(2317)$ and the $X(3872)$. These states have challenged our naive quark model understanding of the mesonic spectra and forced many theorists to revisit their prior assumptions. Since their discovery, many models have been proposed to explain these new states and perhaps predict more states like them. One approach includes virtual meson loops in the constituent quark model and calculates the resulting mass corrections as a possible solution to inconsistency of the new mesons and previous models. In this work we study this model in detail, examining the various approximations and assumptions made in order to determine its viability as an answer to this problem. To do so we use the Quark Pair Creation model for the strong decay vertex. We then apply the model to various mesonic spectra to determine its viability.
\end{abstract}




\section{Acknowledgments}

Firstly, I would like to thank my supervisor Prof. Godfrey for the depth of his knowledge on hadron spectroscopy and his patience in teaching me. I also wish to thank Dr. Ken Moats, whose code laid the foundation of my calculations and was of great assistance when I needed help with the details of the decay model, and my parents as it was only with their support that I was free to focus on my research.

Finally I would like to thank my fellow officemates and graduate students for putting up with my constant stream of jokes and for helping me understand the material by listening to me go on endlessly about mesons. 


\section{Table of Contents}

Abstract $\quad$ iii

Acknowledgments $\quad$ iv

Table of Contents $\quad$ v

List of Tables vii

List of Figures viii

1 Introduction $\quad 1$

1.1 Brief Introduction to QCD . . . . . . . . . . . . . . . . . 2

1.2 Constituent Quark Model . . . . . . . . . . . . . . . . 7

1.3 What Comes Next . . . . . . . . . . . . . . . 14

2 Coupled Channel Model 15

2.1 Master Equation and the Loop Mass Function . . . . . . . . . . . . . 16

2.2 Structure of the Loop Mass Function, $\Omega$. . . . . . . . . . . . . 20

2.3 The OZI Rule and the Quark Pair Creation Model . . . . . . . . . . 24

2.4 Short Note on Other Models of Strong Decay . . . . . . . . . . . . 31

2.4.1 Cornell Model of Strong Decay . . . . . . . . . . . . . . . . . 31

2.4.2 Scalar Confining Interaction and One Gluon Exchange Model 33

3 Detailed Study of the Overlap Integrals 36

3.1 Parameter Determination . . . . . . . . . . . . . . 36

3.2 General Structure of the Momentum Overlap Integral . . . . . . . . . 39

3.2.1 Single $\beta$ and a form factor . . . . . . . . . . . . . . . . . . 40

3.2.2 Unequal $\beta$ and unequal mass ... . . . . . . . . . . . 44 
3.3 Summary of Parameter Dependence . . . . . . . . . . . . . . . 47

4 Bottomonium Spectrum $\quad 49$

4.1 Coupling to intermediate low lying S-waves . . . . . . . . . . . . . 50

$4.2 \Upsilon(1 S)$ as Test of Convergence . . . . . . . . . . . . . . . 58

5 Charm-Strange Spectrum $\quad 63$

5.1 Naive predictions . . . . . . . . . . . . . . . 63

5.2 Charm Strange Mesons and the Continuum . . . . . . . . . . . 68

$\begin{array}{llr}6 & \text { Conclusion } & 78\end{array}$

Appendix A Anuglar Momentum, Colour, Flavour, Spin... 82

A.1 Clebsch-Gordan Coefficients and the Wigner $n j$ Symbols . . . . . . . 82

A.2 Evaluating the Colour, Flavour and Spin Overlaps for QPC Model of Meson Decay . . . . . . . . . . . . . . . . . . . . . . . . . . 83

A.2.1 Colour Overlap . . . . . . . . . . . . . . . . . . . 84

A.2.2 Flavour Overlap . . . . . . . . . . . . . . . . . . . . . . 85

A.2.3 Spin Overlap . . . . . . . . . . . . . . . . . . . . . . . . . . . 87

$\begin{array}{lll}\text { Appendix B Field Theory Conventions } & 89\end{array}$

Appendix C Complete Bottomonium Results $\quad 91$

$\begin{array}{ll}\text { Bibliography } & 111\end{array}$ 


\section{List of Tables}

1.1 Quark Properties Table . . . . . . . . . . . . . . . . 5

$3.1{ }^{3} P_{0}$ model parameters . . . . . . . . . . . . . . . . . . . . . 39

4.1 Ground State S-Wave B-Mesons . . . . . . . . . . . . . . . 50

4.2 Properties of Sample Bottomonium States _ . . . . . . . . . . 51

4.3 Mass Corrections to $1 S$ and $1 P b \bar{b}$ States from $1 S$ Bottom and Bottom Strange Mesons . . . . . . . . . . . . . . . . . 53

4.4 Low Lying Bottomonium Coupling to $1 S+1 S$ Intermediate States . 55

4.5 Comparison between full $1 S+1 S$ intermediate loops of this work and other published results .................. . 56

4.6 Radially Excited S-wave Contributions to $\Upsilon(1 S)$ in $1 \beta$ Scheme . . . . 59

4.7 Radially Excited P-wave Contributions to $\Upsilon(1 S)$ in $1 \beta$ Scheme . . . 59

4.8 Radially Excited S-wave Contributions to $\Upsilon(1 S)$ in $1 \beta+r_{q}$ Scheme . 60

4.9 Radially Excited P-wave Contributions to $\Upsilon(1 S)$ in $1 \beta+r_{q} \ldots \ldots 66$

4.10 Radially Excited S-waves Contributions to $\Upsilon(1 S)$ in $3 \beta+r_{q}$ Scheme 61

4.11 Radially Excited P-wave Contributions to $\Upsilon(1 S)$ in $3 \beta+r_{q}$ Scheme . 62

5.1 Comparison of Masses for Known $D_{s}$ States $\ldots \ldots \ldots$. . . 67

5.2 Charm Strange mesons inputs . . . . . . . . . . . . . . . 69

5.3 Charmed Mesons and kaons Input Data . . . . . . . . . . . . . 71

5.4 Comparison of Total Mass Correction in Charm-Strange Sector . . . . 72

5.5 Coupled Channel Charm Strange with Rescaling Effects . . . . . . . . 73

C.1 Loop corrections to $\Upsilon(1 S)$ including radially and orbitally excited mesons with $1 \beta$ scheme without rescaling. . . . . . . . . . . 92

C.2 Loop corrections to $\Upsilon(1 S)$ including radially and orbitally excited mesons in $1 \beta+r_{q}$ scheme without rescaling. . . . . . . . . . 93

C.3 $1 \beta$ vs $3 \beta$ comparison for $1 S+1 S$ loop mass corrections. $\ldots \ldots \ldots .94$

C.4 $1 \beta+r_{q}$ vs $3 \beta+r_{q}$ comparison for $1 S+1 S$ loop mass corrections. . . 98 


\section{List of Figures}

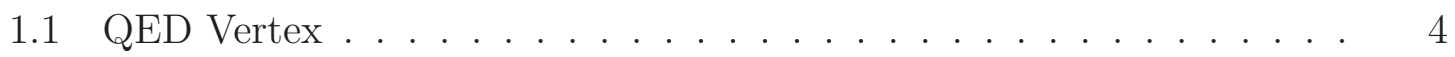

1.2 QCD Vertices . . . . . . . . . . . . . . . . . 6

1.3 Naive Charmonium Wave Functions . . . . . . . . . . . . . . . . . 9

1.3 Naive Charmonium Wave Functions . . . . . . . . . . . . . . . . 10

1.4 Naive Charmonium Spectrum . . . . . . . . . . . . . . . . . . . 12

2.1 Strong Decay Vertex . . . . . . . . . . . . . . . . . 18

2.2 Illustration of loop corrections . . . . . . . . . . . . . . 20

2.3 Denominator of the loop correction function . . . . . . . . . . 22

2.4 OZI violating process $\ldots \ldots \ldots \ldots \ldots$

2.5 Quark rearrangement of strong decay in a meson . . . . . . . . . . 28

3.1 S-wave to S-wave Momentum Overlap Integrals . . . . . . . . . . . 41

3.2 S-wave to S and P-wave Momentum Overlap Integrals . . . . . . . . 42

3.3 Effect of $r_{q}$ on the Shape of the Matrix Element . . . . . . . 43

3.4 Effect of the Constituent Quark Mass on the Matrix Element . . . . . 44

3.5 Overlap integral of $1 S \rightarrow 1 S+1 S$ with all 4 quarks in the vertex . . 45

3.6 Summary Plot of mass, $\beta$ and $r_{q}$ effect on two matrix elements . . . 46

4.1 Mass Correction Curves for $\Upsilon(1 S)$ and $\eta_{b}(1 S) \ldots \ldots \ldots \ldots$

5.1 Charm Strange Spectrum and S-Wave Thresholds . . . . . . . . . 65

5.2 Modified P-wave charm strange spectrum with no rescaling . . . . . 74

5.3 Modified P-wave charm strange spectrum with rescaling $\ldots \ldots .75$

5.4 Mass Correction Function versus Naive Mass for $1 P$ Multiplet of Charm-Strange Mesons . . . . . . . . . . . . . . . . . . 77 


\section{Chapter 1}

\section{Introduction}

The constituent quark model is one of the most successful phenomenological models in particle physics and has been an important guide for hadron physics. From its introduction (independently) by Gell-Mann and Zweig [1, 2] to potential models developed within it [3-7], the constituent quark model has been able to accurately predict various properties of many different hadrons.

While the quark model is still very good at modeling hadrons, over the past decade new states have been discovered that challenge all quark model calculations [8, 9]. The first of these states was the $D_{s}(2317)$, found in the charm-strange spectrum [10] with a mass much lighter and a width significantly narrower than any quark model predictions [8]. This was followed by the discovery of the more famous exotic meson, the $X$ (3872), found in the charmonium spectrum [11]. No quark model had predicted such a state. While these two are great examples, more and more states have since been discovered (refer to Ref. [12] for a comprehensive list of suspected

exotic states). As a result, the question has become: why would a previously (and currently) successful model misfire on a few states? Why would a state be predicted to be highly unstable with a large decay width only to be observed with a vanishingly small width and be quite stable? 
In this work we will study a coupled channel framework introduced to bring these rogue states back into the quark model fold. The coupled channel model posits that through coupling to the nearby strong decay thresholds, whether open or closed, naive quark model states receive mass corrections which could explain the strange experimental observations. To do so, we will choose a well-known and frequently applied decay model, the Quark Pair Creation (QPC) Model to calculate mass shifts induced by virtual meson loops to two mesonic spectra. The coupled channel framework and the QPC choice for modelling the interaction vertex have a long history in quark model with a rather substantial volume of results and calculations published in the literature (which we will cover in chapters 4 and 5). Thus our aim is to study the details of this model and its sensitivity to the assumptions made in calculations. Ultimately we wish to determine whether this is a viable method to study and account for the exotic states observed and whether previous calculations are reliable.

We begin our study by first briefly discussing Quantum Chromodynamics, the theory of the strong interactions, before reviewing the constituent quark model in this chapter.

\subsection{Brief Introduction to QCD}

Quantum ChromoDynamics (QCD) is the quantum field theory of the strong interactions and part of the currently reigning theory of matter, the Standard Model of Particle Physics (SM for short). QCD was historically born out of the Quark Model and concerns itself with 6 quarks (the fermions of the theory, similar to the electron in Quantum Electrodynamics)[13] which are charged under the strong force. The charge in QCD is dubbed "colour" [14] and much like how the electron carries a unit of "electric" charge, quarks carry the colour charge with their anti-particles carrying 
the anti-colour charge. It should be noted that quarks are still charged under all other SM forces. In QCD, the carriers of the force are the eight gluons [15] which are massless bosons.

Quarks are organized into 3 so-called "generations" with each quark having its own "flavour"

$$
\left(\begin{array}{c}
\mathrm{up}, \mathrm{u} \\
\text { down, d }
\end{array}\right),\left(\begin{array}{c}
\text { charm, c } \\
\text { strange, s }
\end{array}\right),\left(\begin{array}{c}
\text { top, } \mathrm{t} \\
\text { bottom, b }
\end{array}\right) \text {. }
$$

QCD respects flavour conservation which means within the framework of QCD, quarks can not mutate to other quarks or change generation. This is not the case in Electroweak theory in SM. For example, a bottom quark can emit a virtual $W^{-}$boson and turn into a charm quark. Such interactions are beyond the scope of this work.

The colour charge that was mentioned before comes in three states (unlike QED which has only one) usually denoted as column vectors [13]

$$
(\text { red, } r)=\left(\begin{array}{l}
1 \\
0 \\
0
\end{array}\right),(\text { green, } \mathrm{g})=\left(\begin{array}{l}
0 \\
1 \\
0
\end{array}\right),(\text { blue }, \mathrm{b})=\left(\begin{array}{l}
0 \\
0 \\
1
\end{array}\right) \text {. }
$$

The Lagrangian of the SM is invariant under rotations of the symmetry group for colour. This means that all quarks couple to gluons with equal strength and no quark is favoured over the other [13]. So far, QCD and QED sound quite similar to each other. However, this is where the paths diverge.

While the carrier of the electromagnetic force in QED, the photon, is not charged itself, the gluon is charged under QCD [13]. Thus, while in QED the photon cannot 


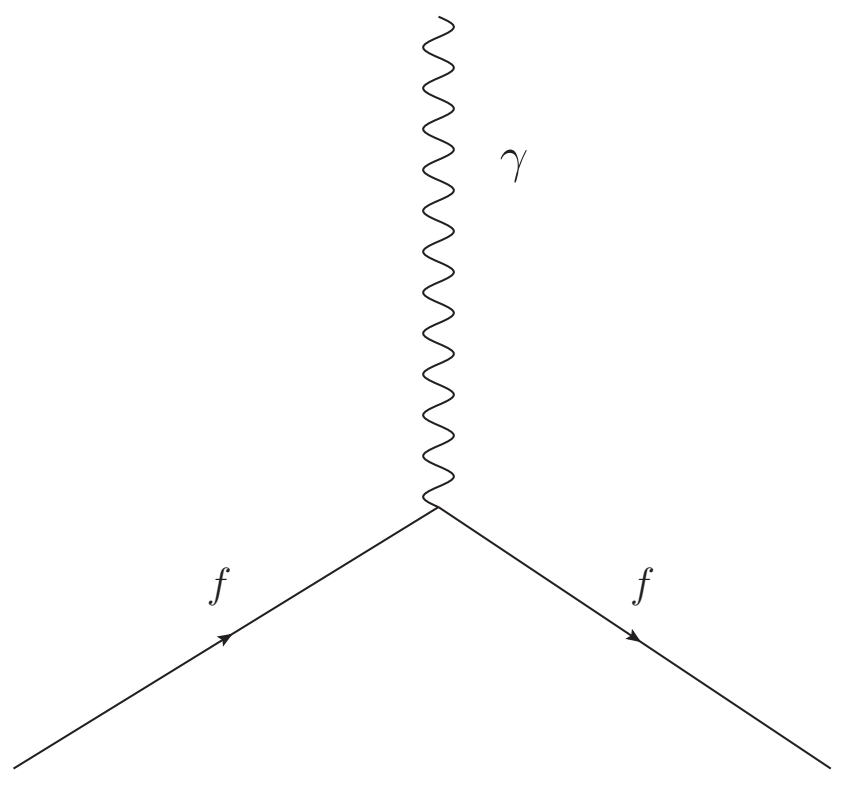

Figure 1.1: The only allowed vertex in QED. $f$ stands for "fermion" which can be any of the charged fermions in the standard model: $e^{ \pm}, \mu^{ \pm}, \tau^{ \pm}, u, d, c, s, t, b$. All Feynman diagrams in QED must be made from different combinations of this vertex.

emit another photon, in QCD a gluon can emit more gluons. Another interesting aspect is the strength of $\alpha_{s}$, the analog of the fine structure constant in QED. The value of the fine structure constant, $\alpha$, is $\approx \frac{1}{137}[12]$ at low energies and the fact that this value is small is the reason why perturbation theory is so successful in QED. In QED each interaction vertex, as shown in Fig. 1.1, contributes an $\alpha$. In the low energy regime, we only have to consider diagrams made of a small number of vertices (we need at least two vertices to create the simplest QED process) and higher order corrections which include more vertices and therefore larger powers of $\alpha$ are suppressed 
by its value. As the energy scale of the interaction (for example in electron-positron scattering or some such process) grows, so does $\alpha$ and more complicated diagrams need to be considered since they are no longer suppressed by the "small" value of $\alpha$. These digrams will have more than two copies of the Fig. 1.1 vertex. However, this increase in the magnitude of $\alpha$ is slow and even at high energy scales (at the mass of the $\mathrm{W}$-boson for example, which is $\approx 80 \mathrm{GeV}) \alpha$ rises to $\approx \frac{1}{128}$ [12]. Thus, perturbation theory remains useful in QED up to very high energy scales.

As was mentioned before, unlike the photon, the gluon in QCD is also charged under the strong interaction. Unlike in QED, it is in the high energy regime that $\alpha_{s}$ of QCD is small and allows for the use of perturbation theory. This phenomenon, the smallness of $\alpha_{s}$ at high energy, is called asymptotic freedom and since high energies translate to small distances, one could think that within a composite particle made of quarks, the quarks move as if they are free with minimal interaction. At smaller

Table 1.1: Properties of the six known quarks. Current masses are from Ref. [12]. The constituent mass is model dependent however masses provided here are "typical" values used in many calculations. The top quark does not get a constituent mass as it decays too rapidly to form bound states. In this work we use natural units, meaning $c=\hbar=1$.

\begin{tabular}{cccc}
\hline Quark & Electric Charge & Current Mass & Constituent Mass \\
\hline up & $+\frac{2}{3}$ & $2.2_{-0.4}^{+0.6} \mathrm{MeV}$ & $330 \mathrm{MeV}$ \\
\hline down & $-\frac{1}{3}$ & $4.7_{-0.4}^{+0.5} \mathrm{MeV}$ & $330 \mathrm{MeV}$ \\
\hline charm & $+\frac{2}{3}$ & $1.28 \pm 0.03 \mathrm{GeV}$ & $1.5 \mathrm{GeV}$ \\
\hline strange & $-\frac{1}{3}$ & $96_{-4}^{+8} \mathrm{MeV}$ & $550 \mathrm{MeV}$ \\
\hline top & $+\frac{2}{3}$ & $173.1 \pm 0.6 \mathrm{GeV}$ & - \\
\hline bottom & $-\frac{1}{3}$ & $4.18_{-0.03}^{+0.04} \mathrm{GeV}$ & $4.7 \mathrm{GeV}$ \\
\hline
\end{tabular}



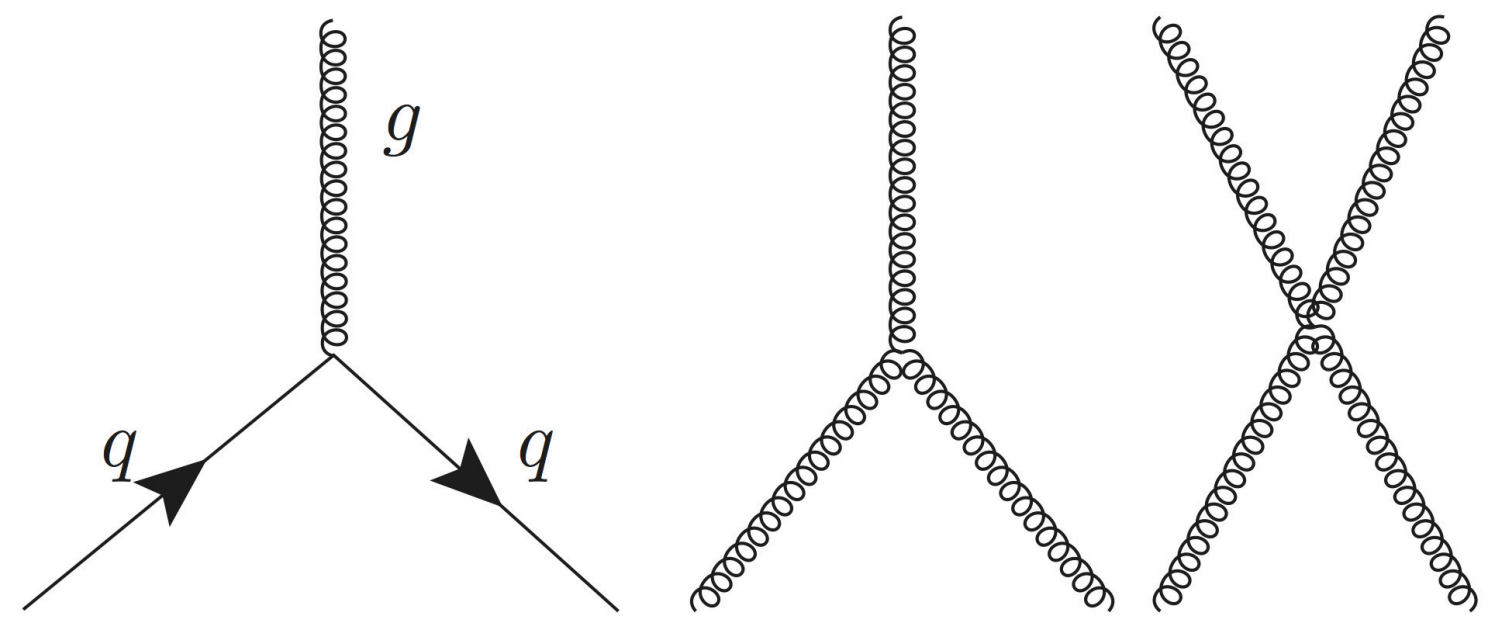

Figure 1.2: Allowed vertices in QCD. $g$ and $q$ stand for gluon and quark (of course q can also be an antiquark). All Feynman diagrams in QCD are made of some combination of these three vertices.

energy scales which translates to larger separation, another phenomenon kicks in: that of confinement which is the reason why no free quarks have ever been observed. For larger distances, $\alpha_{s}$ grows and eventually causes perturbation theory to fail. While this is an amazing effect, it does pose the conundrum that in the energy scale that hadrons are formed, the energy scale that is important to us, Feynman calculus fails. It is this failure that has made QCD such a difficult theory to use and apply. Putting these two phenomena together paints a picture of the world according to QCD that is radically different from other theories or our everyday life experience which is dominated by $1 / r$ interactions; in QCD the further away two objects are the more strongly they interact.

At the moment the most promising and successful method of solving problems in QCD in the low energy regime is Lattice QCD which is a numerical technique. However, the method is extremely computationally intensive, difficult to implement and while it has come a long way it is still limited in its applicability. Therefore one can make the case that until Lattice QCD is fully developed, quark models still offer 
good insights into physics of strongly interacting particles. The constituent quark model is the topic we will discuss next and it, not QCD, will be the focus of this work.

\subsection{Constituent Quark Model}

The Quark Model takes hadrons (particles observed in experiments that are produced via the strong force such as protons, neutrons and so on) to be composites of elementary particles called "quarks". Quarks were introduced in the quark model to be fermions with fractional electric charge $[1,2]$ and hadrons were divided into two main groups

- Mesons are bound states of a quark and an anti-quark

- Baryons are bound states of three quarks or three anti-quarks.

In this work we will focus on the former. Other combinations of quarks and antiquarks were also considered in the early days of the quark model [16-19] however none were observed and the calculations were not conclusive with different models arriving at different conclusions. The success of potential models also, for the most part, put the issue of multi-quark hadrons on a lower priority level. To this day, pentaquark states are the only type of multiquark hadrons that potentially have been observed [20]. While the $X(3872)$ and other exotics are/have been considered as tetraquark candidates, such assignments are still very model dependent and subject to great controversy.

The quarks of the quark model are identical to the quarks in QCD in all but one property: mass. As can be seen in table 1.1, the constituent quark masses are larger than the "current" quark mass which is what enters in the QCD Lagrangian. 
The difference comes from the fact that within, for example a meson, one does not have just two interacting objects (a quark and an anti quark also known as valence quarks) but also a cloud of gluons and virtual $q \bar{q}$ pairs known as sea quarks. Part of the difficulty of Lattice QCD is in calculating the effect of this quark/gluon cloud. In the quark model, however, effects of this cloud are incorporated in the model by an increase in the mass of the valence quarks. Thus the "constituent" quarks are valence quarks which are dressed with the sea quarks and gluons and thus have an effective mass $[21]$.

In order to calculate mesonic spectra with our constituent quarks, we require a potential model as well as either a relativistic or non-relativistic equation. The simplest quark models use the radial Schrödinger equation [22]

$$
-\frac{1}{2 \mu}\left(\frac{d^{2}}{d r^{2}}+\frac{2}{r} \frac{d}{d r}-\frac{l(l+1)}{r^{2}}\right) R_{n l}(r)+V(r) R_{n l}(r)=E_{n l} R_{n l}(r)
$$

where $r$ is the inter-quark separation, $R_{n l}(r)$ is the radial wave function of the meson, $l$ is the angular momentum between the quark-anti quark pair and finally $E_{n l}$ is the eigenvalue of the equation which we identify with the mass of the meson. $V(r)$ is the $q \bar{q}$ potential and it depends on how we wish to model the system. Among the most successful and popular models is the Cornell Model [5] which postulated the following potential between a quark and an anti-quark

$$
V(r)=-\frac{\kappa}{r}+\frac{r}{a^{2}}+c
$$

In their work, Eichten et al. [5] proposed the potential based on the expected behaviour of QCD in two regimes: at large distances, the potential must be a confining one to account for the confinement phenomenon. At short distances however, it 


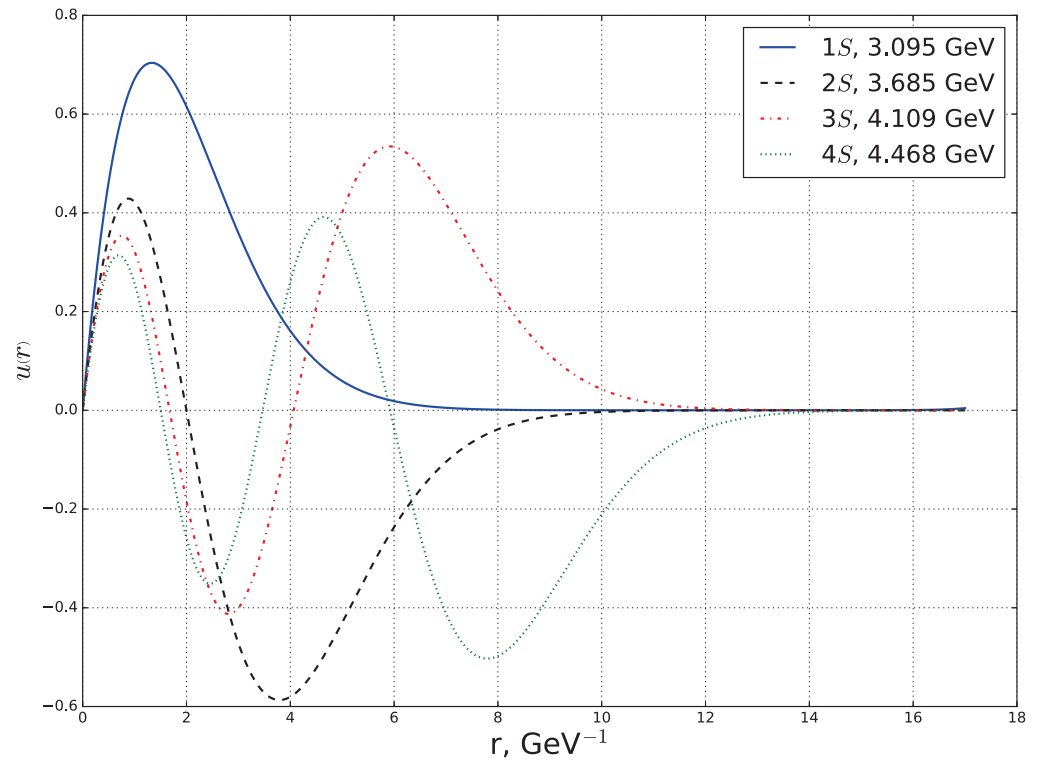

(a) S Waves

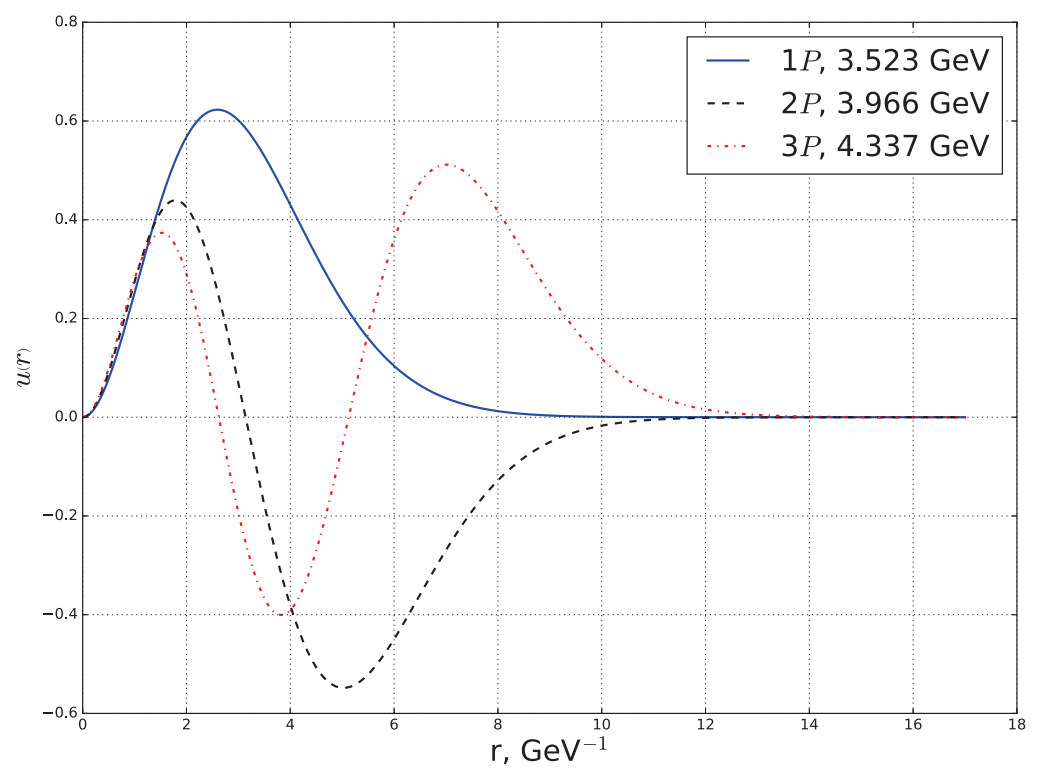

(b) P Waves

Figure 1.3: Numerically calculated wave functions of charmonium using potential parameters of Eq. 1.3. We plot $u(r)$ in $R_{n l}(r)=\frac{u_{n l}(r)}{r}$ for $l=0,1,2,3$ waves $(\mathrm{S}$, $\mathrm{P}, \mathrm{D}$ and $\mathrm{F}$ in spectroscopic notation). The masses (eigenvalues) corresponding to each state are given in the legend. 


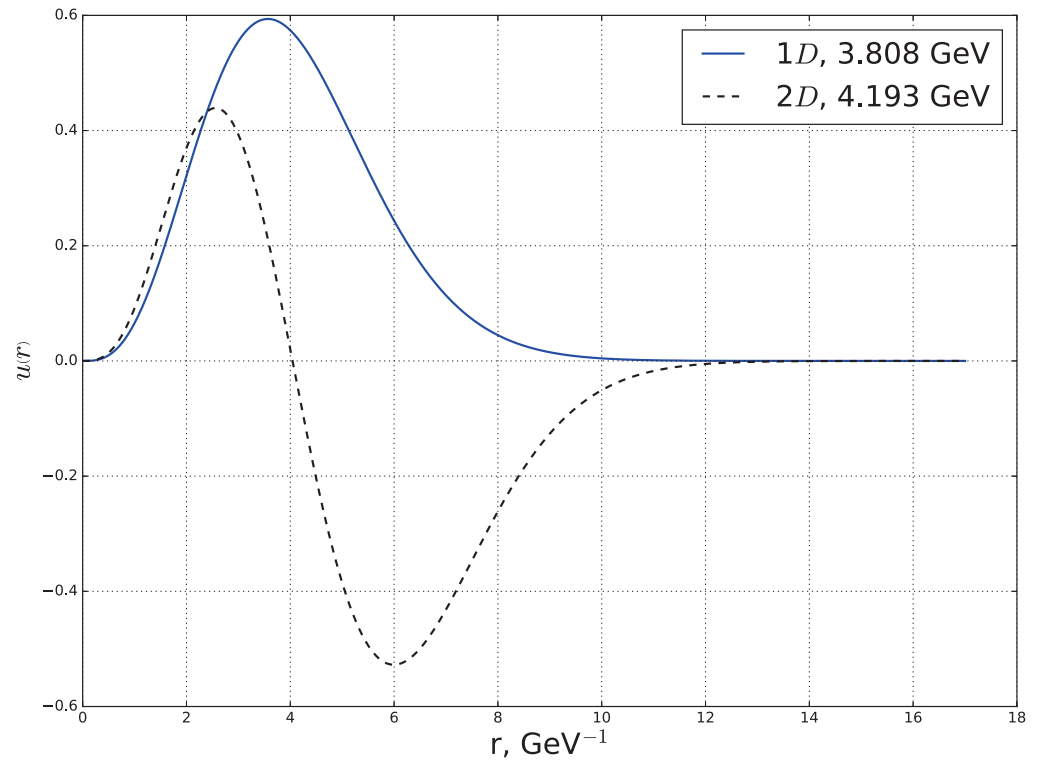

(c) D Waves

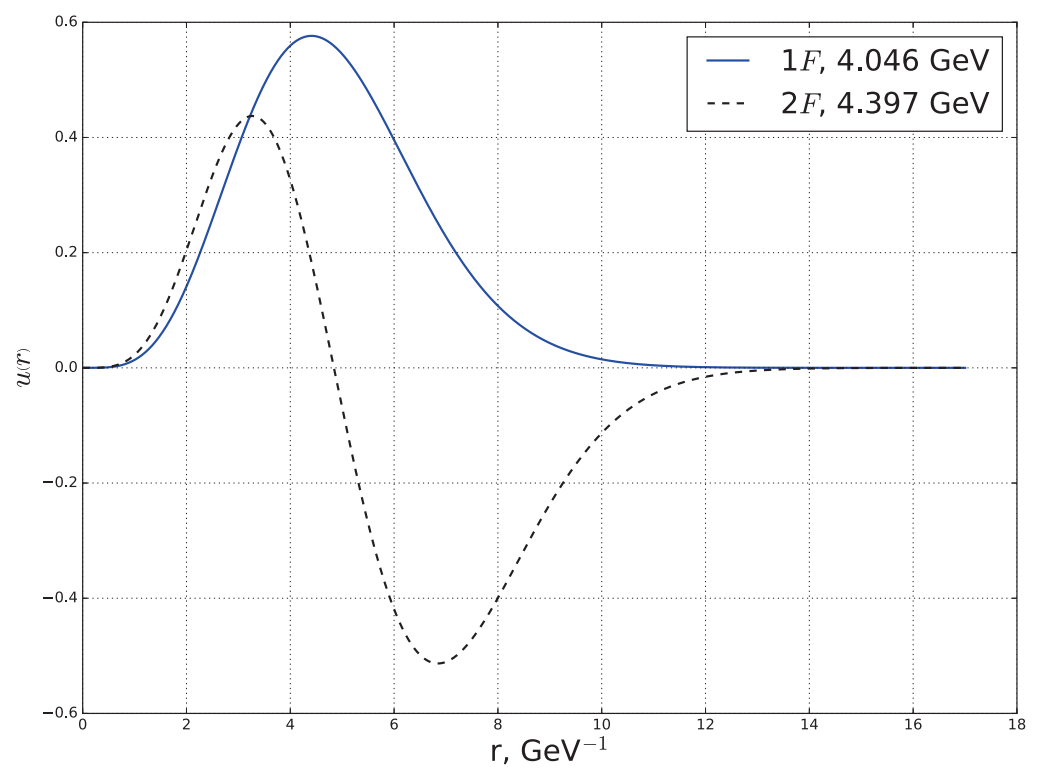

(d) F Waves

Figure 1.3: Numerically calculated wave functions of charmonium using potential parameters of Eq. 1.3. We plot $u(r)$ in $R_{n l}(r)=\frac{u_{n l}(r)}{r}$ for $l=0,1,2,3$ waves (S, $\mathrm{P}, \mathrm{D}$ and $\mathrm{F}$ in spectroscopic notation). The masses (eigenvalues) corresponding to each state are given in the legend. 
should behave like a one-gluon exchange potential which given the fact that the gluon is massless, will look exactly like a Coulomb potential. As such $\kappa$ can be identified with $\alpha_{s}$ but in their work, it was treated as a free parameter. Using the parameters in Ref. [5]

$$
\begin{aligned}
\kappa & =0.52 \\
a & =2.34 \mathrm{GeV}^{-1} \\
m_{c} & =1.84 \mathrm{GeV}
\end{aligned}
$$

we can calculate a naive spectrum for bound states of the charm and anti-charm quark using the Schrödinger equation and a numerical integration technique. As an example, we use the Numerov method [23] to solve for the wave functions of the first few bound states of the $c \bar{c}$ spectrum and plot the results in Fig. 1.3. Since the potential of Eq. 1.2 does not include spin effects, the degeneracies of various multiplets are not lifted which, for a $1 \mathrm{~S}$ multiplet for example, means $1^{3} S_{1}$ and $1^{1} S_{0}$ have the same mass. We also show a sample spectrum plot in Fig. 1.4 using the same parameters ( Eq. 1.3).

Over time, more sophisticated potential models were proposed with much better agreement with experimental results. A useful representative example is the GodfreyIsgur relativized quark model [7]. There the authors make the substitution

$$
\frac{P^{2}}{2 \mu} \rightarrow \sqrt{P^{2}+m_{1}^{2}}+\sqrt{P^{2}+m_{2}^{2}}
$$

where $m_{1}$ and $m_{2}$ are quark masses, $\mu$ is the reduced mass of the system and $P$ is the centre of mass momentum of each quark. Using this relativization and including relativistic smearing effects one can proceed to calculate masses for various mesonic spectra. In the non-relativistic limit, the potential used between the quark and the 


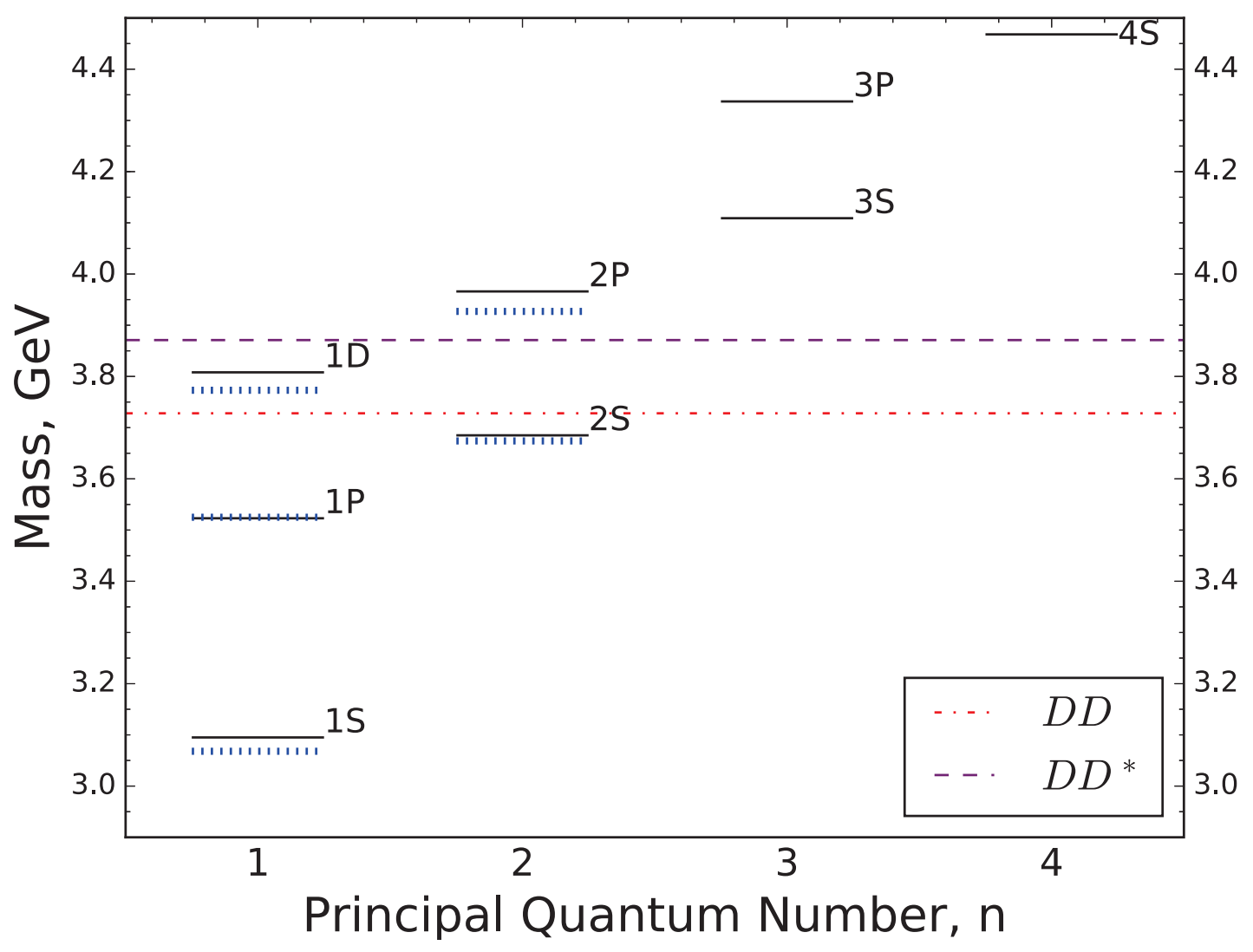

Figure 1.4: Sample spectrum obtained by solving the Schrödinger equation for the potential of Eq. 1.2. Black lines (solid) are the result of our numerical calculation while the blue lines (dotted) are spin averaged experimental data from Ref. [12]. The dashed and dot-dashed lines spanning the plot are the $D D$ and $D D^{*}$ open charm thresholds. The agreement is reasonable given the simplicity of the model. 
anti-quark is given by [7]

$$
V_{i j}(\overrightarrow{\boldsymbol{p}}, \overrightarrow{\boldsymbol{r}}) \rightarrow H_{i j}^{\mathrm{conf}}+H_{i j}^{\mathrm{hyp}+\text { ten }}+H_{i j}^{\mathrm{so}}
$$

where

$$
H_{i j}^{\mathrm{conf}}=-\left[\frac{3}{4} c+\frac{3}{4} b r+\frac{\alpha_{s}(r)}{r}\right] \mathbf{F}_{i}^{a} \cdot \mathbf{F}_{j}^{b}
$$

is the confining interaction,

$$
H_{i j}^{\mathrm{hyp}}=\frac{-\alpha_{s}(r)}{m_{i} m_{j}}\left[\frac{8 \pi}{3} \mathbf{S}_{i} \cdot \mathbf{S}_{j} \delta^{(3)}(r)+\frac{1}{r^{3}}\left[\frac{3 \mathbf{S}_{i} \cdot \mathbf{S}_{j}}{r^{2}}-\mathbf{S}_{i} \cdot \mathbf{S}_{j}\right]\right] \mathbf{F}_{i}^{a} \cdot \mathbf{F}_{j}^{b}
$$

is the colour hyperfine interaction and

$$
\begin{aligned}
H_{i j}^{\mathrm{so}}=\frac{-\alpha_{s}(r)}{r^{3}}\left[\frac{1}{m_{i}}+\frac{1}{m_{j}}\right]\left[\frac{\mathbf{S}_{i}}{m_{i}}+\frac{\mathbf{S}_{j}}{m_{j}}\right] \cdot \mathbf{L}\left(\mathbf{F}_{i}^{a} \cdot \mathbf{F}_{j}^{b}\right) & \\
& -\frac{1}{2 r} \frac{\partial H_{i j}^{\mathrm{hyp}}}{\partial r}\left[\frac{\mathbf{S}_{i}}{m_{i}^{2}}+\frac{\mathbf{S}_{j}}{m_{j}^{2}}\right] \cdot \mathbf{L}
\end{aligned}
$$

is the spin-orbit interaction term. $i$ and $j$ index the quark and the antiquark (in no particular order) and $\alpha_{s}$ is the strong coupling constant which is allowed to run in this model. $\mathbf{S}_{i}$ is the spin of quark $i, m_{i}$ its mass and finally $\mathbf{F}_{i}^{a}$ is its colour wavefunction defined as

$$
\mathbf{F}_{i}^{a}=\left\{\begin{array}{l}
\frac{\lambda_{i}^{a}}{2}, \text { for quarks } \\
\frac{-\lambda_{i}^{* a}}{2}, \text { for anti quarks }
\end{array}\right.
$$

where $\lambda^{a}$ is the $a^{\text {th }}$ Gell-Mann matrix. Since its introduction, this model has been among the most successful constituent quark models. The discussion of the model is beyond the scope of this work and we will only use its results as inputs in chapters 3 and 4 .

Once the numerical wave function is found, spectroscopy in its traditional sense 
can begin: calculating leptonic widths (decays of hadrons to final states involving leptons, elementary particles charged under weak and electromagnetic interaction. $e^{-}$is one such particle), radiative transitions (both magnetic and electric transitions) of hadrons and strong decay calculations. In this work, it is a version of the latter of these calculations that interests us.

\subsection{What Comes Next}

This chapter served as a brief introduction to certain aspects of QCD and some useful terminology in the naive quark model. More importantly, using a very simple potential model we showed the great power of the constituent quark model to predict and describe the various mesonic mass spectra. However, what all these models have in common is a failure to describe the various (suspected) mesonic states mentioned in the beginning of this chapter such as the $D_{s}(2317)$, the $X(3872)$ and so on.

Our aim in what comes next in this work is to study the coupled channel formalism. The proponents of this model claim that the anomalous masses observed for these new states is the result of a coupling between the naive states and two meson channels. We will introduce the formalism of this model in chapter 2 and study the details of the matrix element of our chosen operator in chapter 3. Finally we will consider the application to two specific spectra, bottomonium as bound states of the b-quark and the anti-b quark and the charm-strange spectrum in chapters 4 and 5, respectively. In our studies, we look for how changing the various details of the model distorts the naive potential model predictions and if the formalism can successfully describe the mesonic spectra particularly the exotic states. Finally we state our main conclusions in chapter 6 and suggest possible topics to explore in the future. 


\section{Chapter 2}

\section{Coupled Channel Model}

We begin our discussion in this chapter by revisiting the naive sample spectrum in the

previous one. There we solved the non-relativistic Schrödinger equation for a given $q \bar{q}$ potential to find eigenvalues and eigenfunctions. Such calculation assumes that in our system, we can always clearly define the mass of a bound state or in other words: all our states are either perfectly stable or have long life times.

However, we know from theoretical calculations and experimental observation that most states lie above the two body decay threshold. Furthermore, these states have small life times (i.e. large decay widths) which clearly negates the naive assumption of stability. These facts paint a rather more complex picture of meson spectroscopy than what we saw in the previous chapter. They point towards the idea that in order to make our calculations more realistic and give a better explanation of what is observed, we have to allow for coupling between naive states and decay channels. Since large decay widths imply strong coupling between the decaying meson and the products, it also stands to reason that virtual loops of mesons could be important [24].

The potential effects of such couplings were recognized in the early days of both the quark model and QCD. However they were largely ignored due to the tremendous 
success of non-relativistic quark models, the relativized quark model later on and lack of computing power. A few groups calculated mass shifts as well as widths [25-29] while others used more and more sophisticated non-relativistic potential models or relativized quark models to obtain their $q \bar{q}$ masses and calculated decay widths using models of strong decay, if the state was located above threshold[7, 30].

Discovery of states such as the $D_{s}^{ \pm}(2317)$ [10] as well as advances in computing power ignited new interest in accounting for mass shifts as well as widths in calculations in hadronic spectra. In the next section, we shall give a general derivation to illustrate the idea and refer the reader to Refs. $[5,26]$.

\subsection{Master Equation and the Loop Mass Function}

The aim of this section is to derive an equation describing the mass of a naive state and the corrections that result from coupling to the continuum. We begin by writing the physical meson's state vector which now includes a higher order Fock space term. Here the calculation will closely follow that of Refs. [31, 32].

$$
|\Psi\rangle=\left(\begin{array}{c}
\sum_{\alpha} n_{\alpha}\left|\phi_{\alpha}\right\rangle \\
\sum_{\beta} \int \mathrm{d}^{3} P \Theta_{\beta}(\overrightarrow{\boldsymbol{P}})\left|M_{1}^{\beta}(\overrightarrow{\boldsymbol{P}}) M_{2}^{\beta}(-\overrightarrow{\boldsymbol{P}})\right\rangle
\end{array}\right)
$$

where $\left|\phi_{\alpha}\right\rangle$ is the $\alpha^{t h} q \bar{q}$ wave function, $\left|M_{1}(\overrightarrow{\boldsymbol{P}}) M_{2}(-\overrightarrow{\boldsymbol{P}})\right\rangle$ is the $\beta^{\text {th }}$ two meson chan-

nel with $n_{\alpha}$ and $\Theta_{\beta}(\overrightarrow{\boldsymbol{P}})$ their respective probability amplitudes. $\overrightarrow{\boldsymbol{P}}$ is the momentum of each meson (one will have an opposite direction) in the centre of mass frame.

The wave functions of $q \bar{q}$ and the two mesons obey the following orthonormality 
conditions

$$
\begin{aligned}
\left\langle\phi_{\alpha} \mid \phi_{\gamma}\right\rangle & =\delta_{\alpha, \gamma} \\
\left\langle M_{1}^{\sigma}\left(\overrightarrow{\boldsymbol{P}^{\prime}}\right) M_{2}^{\sigma}\left(-\overrightarrow{\boldsymbol{P}}^{\prime}\right) \mid M_{1}^{\beta}(\overrightarrow{\boldsymbol{P}}) M_{2}^{\beta}(-\overrightarrow{\boldsymbol{P}})\right\rangle & =\delta_{\sigma, \beta} \delta^{3}\left(\overrightarrow{\boldsymbol{P}}-\overrightarrow{\boldsymbol{P}}^{\prime}\right) \\
\langle\Psi \mid \Psi\rangle & =1 .
\end{aligned}
$$

The Hamiltonian matrix governing the system is given by

$$
H=\left(\begin{array}{cc}
H_{C} & \mathcal{V} \\
\mathcal{V} & H_{M M}
\end{array}\right)
$$

where $H_{C}$ is the Hamiltonian of the $q \bar{q}$ sector whose eigenvectors are the $\left|\phi_{\alpha}\right\rangle$

$$
H_{C}\left|\phi_{\alpha}\right\rangle=m_{\alpha}\left|\phi_{\alpha}\right\rangle
$$

with $m_{\alpha}$ being the mass of the naive state $\alpha$ or the "bare" mass. The two meson Hamiltonian, $H_{M M}$, gives the total energy of the two meson channel, which we will take to be the relativistic energy

$$
\begin{aligned}
H_{M M}\left|M_{1}^{\beta}(\overrightarrow{\boldsymbol{P}}) M_{2}^{\beta}(-\overrightarrow{\boldsymbol{P}})\right\rangle & =\left(\sqrt{P^{2}+m_{1 \beta}^{2}}+\sqrt{P^{2}+m_{2 \beta}^{2}}\right)\left|M_{1}^{\beta}(\overrightarrow{\boldsymbol{P}}) M_{2}^{\beta}(-\overrightarrow{\boldsymbol{P}})\right\rangle \\
& =\left(E_{1}(\overrightarrow{\boldsymbol{P}})+E_{2}(-\overrightarrow{\boldsymbol{P}})\right)\left|M_{1}^{\beta}(\overrightarrow{\boldsymbol{P}}) M_{2}^{\beta}(-\overrightarrow{\boldsymbol{P}})\right\rangle \\
& =E_{M_{1} M_{2}}^{\beta}(\overrightarrow{\boldsymbol{P}})\left|M_{1}^{\beta}(\overrightarrow{\boldsymbol{P}}) M_{2}^{\beta}(-\overrightarrow{\boldsymbol{P}})\right\rangle .
\end{aligned}
$$

$H_{M M}$ can also contain interactions between the two mesons. In this work, however, we ignore such effects. 


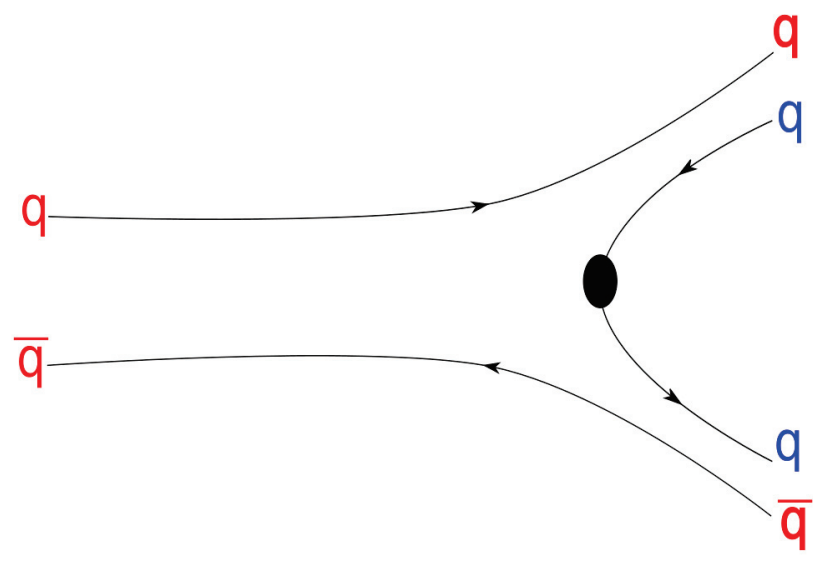

Figure 2.1: Illustration of the matrix element $\left\langle M_{1}^{\beta}(\overrightarrow{\boldsymbol{P}}) M_{2}^{\beta}(-\overrightarrow{\boldsymbol{P}})|\mathcal{V}| \phi_{\alpha}\right\rangle$. The $\mathcal{V}$ potential creates a quark and anti-quark pair (in blue, the inner lines) which then gets rearranged, along with the original pair (in red, the outer lines), into outgoing mesons. The quantum numbers of the created pair and their spatial details depend on the model being used.

The off-diagonal element of the Hamiltonian matrix is the transition potential. It describes the transition of a $q \bar{q}$ state into a meson-meson state and vice versa. The operator and functional form of $\mathcal{V}$ can, in principle, be derived from QCD. However due to the difficult nature of calculations in QCD, the exact structure of the operator, its functional form or the angular momentum and spin configuration of the created quarks is not known. As such, we rely on models in order to calculate widths and mass corrections. For now we will treat $\mathcal{V}$ as a general operator that creates a quark and anti-quark pair, with a graphical representation of what $\mathcal{V}$ does given in Fig. 2.1. Applying $H$ to our physical state $|\Psi\rangle$ now gives

$$
H|\Psi\rangle=M_{\Psi}|\Psi\rangle
$$

with $M_{\Psi}$ taken to be the physical mass of the state $|\Psi\rangle$. Applying Eq. 2.3 explicitly 
to Eq. 2.1 gives

$$
H|\Psi\rangle=\left(\begin{array}{c}
\sum_{\alpha} n_{\alpha} m_{\alpha}\left|\phi_{\alpha}\right\rangle+\sum_{\beta} \int \mathrm{d}^{3} P \Theta_{\beta}(\overrightarrow{\boldsymbol{P}}) \mathcal{V}\left|M_{1}^{\beta}(\overrightarrow{\boldsymbol{P}}) M_{2}^{\beta}(-\overrightarrow{\boldsymbol{P}})\right\rangle \\
\sum_{\alpha} n_{\alpha} \mathcal{V}\left|\phi_{\alpha}\right\rangle+\sum_{\beta} \int \mathrm{d}^{3} P \Theta_{\beta}(\overrightarrow{\boldsymbol{P}}) E_{M_{1} M_{2}}^{\beta}(\overrightarrow{\boldsymbol{P}})\left|M_{1}^{\beta}(\overrightarrow{\boldsymbol{P}}) M_{2}^{\beta}(-\overrightarrow{\boldsymbol{P}})\right\rangle
\end{array}\right)
$$

which is a system of coupled equations. Using the second of the normalization conditions, Eq. 2.2b, we solve for $\Theta_{\beta}(\overrightarrow{\boldsymbol{P}})$

$$
\Theta_{\beta}(\overrightarrow{\boldsymbol{P}})=\frac{\left.\sum_{\alpha} n_{\alpha}\left\langle M_{1}^{\beta}(\overrightarrow{\boldsymbol{P}}) M_{2}^{\beta}(-\overrightarrow{\boldsymbol{P}})|\mathcal{V}| \mid \phi_{\alpha}\right\rangle\right\rangle}{M_{\Psi}-E_{M_{1} M_{2}}^{\beta}(\overrightarrow{\boldsymbol{P}})} .
$$

Substituting for $\Theta$ into the first equation of Eq. 2.7 and using normalization conditions again, we arrive at

$$
M_{\Psi}=\frac{\sum_{\alpha}\left|n_{\alpha}\right|^{2} m_{\alpha}}{\sum_{\alpha}\left|n_{\alpha}\right|^{2}}+\sum_{\beta} \int \mathrm{d}^{3} P \frac{\sum_{\alpha}\left|n_{\alpha}\right|^{2}\left|\left\langle M_{1}^{\beta}(\overrightarrow{\boldsymbol{P}}) M_{2}^{\beta}(-\overrightarrow{\boldsymbol{P}})|\mathcal{V}| \phi_{\alpha}\right\rangle\right|^{2}}{\sum_{\alpha}\left|n_{\alpha}\right|^{2}\left(M_{\Psi}-E_{M_{1} M_{2}}^{\beta}(\overrightarrow{\boldsymbol{P}})\right)} .
$$

If we take our physical state to be made of just one dominant $q \bar{q}$ component, $\left|\phi_{0}\right\rangle$, we can rewrite Eq. 2.9 to obtain the master equation

$$
M_{\Psi}=m_{0}+\Omega\left(M_{\Psi}\right)
$$

with the correction function, $\Omega(E)$, defined as

$$
\Omega(E)=\sum_{\beta} \int P^{2} \mathrm{~d} P \frac{\left|\left\langle M_{1}^{\beta}(\overrightarrow{\boldsymbol{P}}) M_{2}^{\beta}(-\overrightarrow{\boldsymbol{P}})|\mathcal{V}| \phi_{0}\right\rangle\right|^{2}}{E-E_{M_{1} M_{2}}^{\beta}(\overrightarrow{\boldsymbol{P}})} .
$$

The $\beta$ sum runs over all possible meson-meson intermediate channels. That is to 


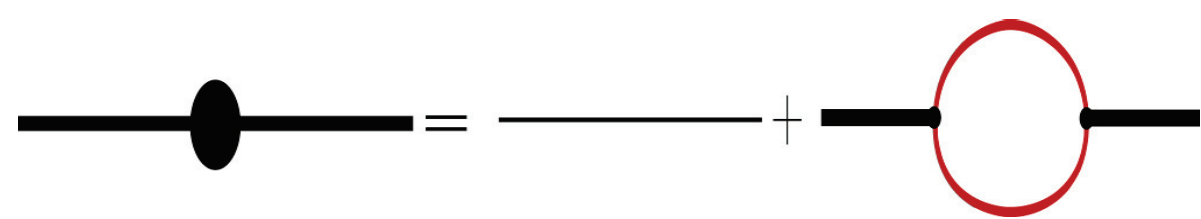

Figure 2.2: Pictorial representation of the mass equation 2.10. The physical state's mass is the sum of the naive state's mass and the loop corrections.

say if for some $\beta, \phi_{0} \rightarrow M_{1}^{\beta}+M_{2}^{\beta}$ is not prohibited by flavour, charge or spin and angular momentum conservation then said channel is included in the sum. However, in practice, most studies on meson loop effects have limited the calculation to ground state S-waves mesons.

\subsection{Structure of the Loop Mass Function, $\Omega$}

Given the central role and importance of Eq. 2.10, we believe it is worthwhile to take a closer look at $\Omega(E)$ and study its behaviour. As stated before, the matrix element $\left\langle M_{1} M_{2}|\mathcal{V}| \phi\right\rangle$ should, in principle, be calculated from QCD. We have to make due with models, however, given the computational complexity of QCD and lack of theoretical progress. We will discuss the Quark Pair Creation Model in the next section but for now, we can keep the discussion general while still learning more about meson loops effect.

The matrix elements, generally, can be factorized into the angular momentum recoupling coefficients characterized by a series of Clebsch-Gordan, Wigner 3j, 6j and 9j symbols (see Appendix A) which then are multiplied by the spatial part [33]. So for a general operator, T, we can write

$$
\left\langle M_{1} M_{2}|T| \phi\right\rangle=C_{J_{M_{1}}, L_{M_{1}}, S_{M_{1}}}^{J_{\phi}, L_{1}, S_{\phi}} \mathcal{I}\left(\overrightarrow{\boldsymbol{P}}, m_{1}, m_{2}, m_{3}, m_{4}\right)
$$

where J, L and S are total spin, angular momentum and spin of the specified particle 
and $C$ is shorthand for all coefficients resulting from the recoupling calculations. $\mathcal{I}\left(\overrightarrow{\boldsymbol{P}}, m_{1}, m_{2}, m_{3}, m_{4}\right)$ are momentum overlap (or coordinate space overlap) integrals of the three wave functions involved, which are functions of the constituent quark masses as well as the momentum of the two daughter mesons. The exact shape and scale of these functions or whether they can be integrated analytically or not, depends on the model that is chosen. Regardless, $\mathcal{I}\left(\overrightarrow{\boldsymbol{P}}, m_{1}, m_{2}, m_{3}, m_{4}\right)$ can be seen as a function of momentum, taking a value for $\mathrm{P}$ and returning a value for the matrix element, once the recoupling coefficients are taken into account. We will discuss $\mathcal{I}\left(\overrightarrow{\boldsymbol{P}}, m_{1}, m_{2}, m_{3}, m_{4}\right)$ further later on.

Moving on to the rest of Eq. 2.11, we observe that the numerator is a positive, real number. Therefore, what sets the sign of the $\Omega(E)$ function is the denominator which is, in a sense, an expression of conservation of energy in a two body decay.

In Fig. 2.3 we plot the denominator of Eq. 2.11 versus momentum for a hypothetical threshold (set at $M_{1}+M_{2}=4$ ) for a series of different energy values (which in our work can be seen as the mass). As it can be seen, the expression is strictly negative below threshold. For $E \geq M_{1}+M_{2}$, the curve will cross the P-axis at the two body decay momentum where the parent state, A, has enough mass to be able to decay to $B+C$. In other words, the decay channel opens when the mass of the parent state is greater than the sum of the masses of the daughters. As the energy is increased, the location of this crossing moves towards larger and larger $\mathrm{P}$ values. At this point we can include the expected behaviour of the wave-functions in order to deduce the asymptotic behaviour of the momentum overlap integral. The wave-function of a meson is well-behaved. This means that at larger and larger momentum values, we expect the momentum overlap integral to go to zero, sufficiently quickly. Taking this point into account, we can deduce that if our naive state is located above threshold 


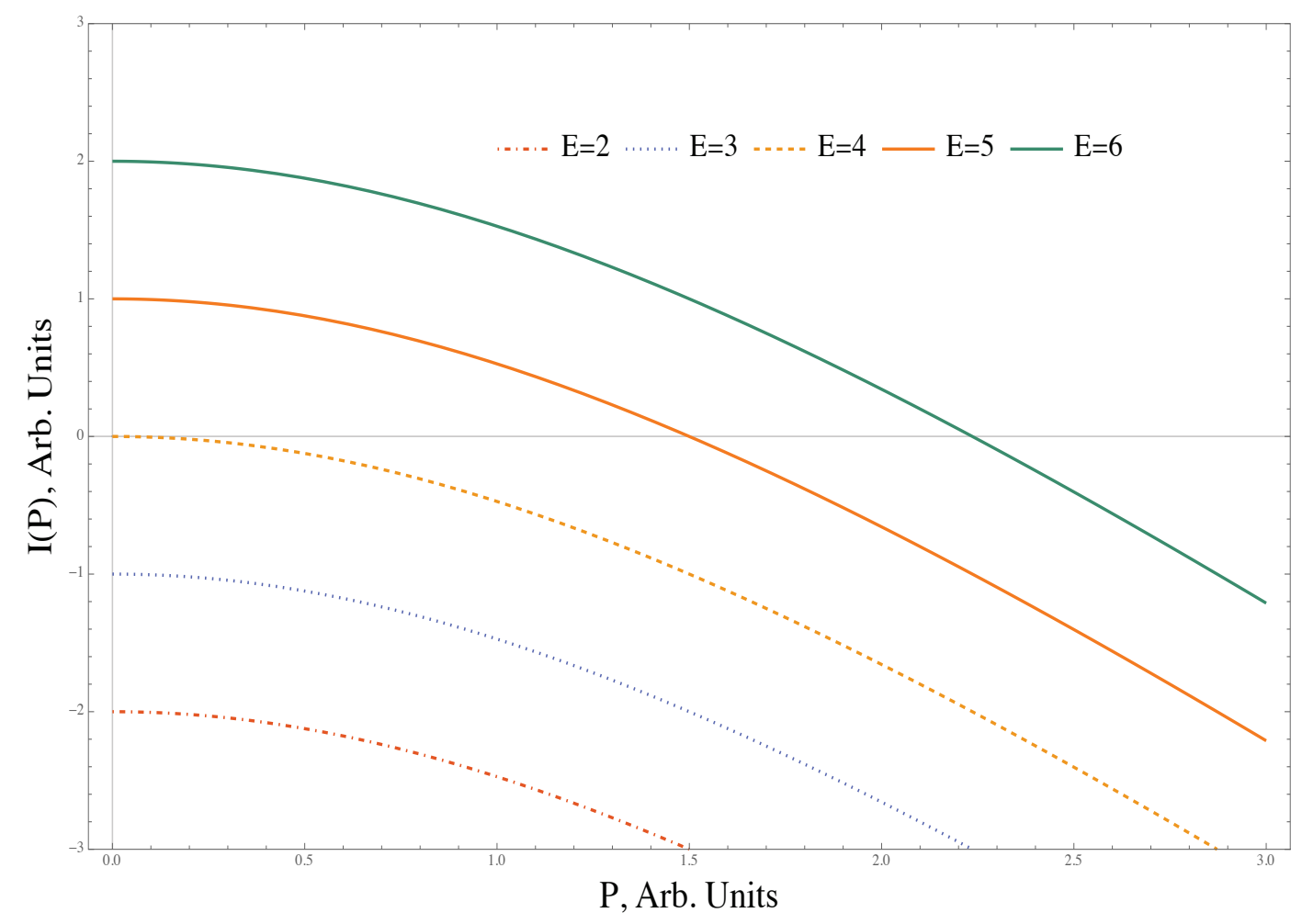

Figure 2.3: Plot of the denominator of Eq. 2.11 for a test threshold plotted at various energy values below, at and above threshold. Threshold is set at $E=4$.

(i.e. $m_{0} \geq M_{1}^{\beta}+M_{2}^{\beta}$ for some $\beta$ ) then it is possible for the mass correction from that channel to be positive if $m_{0}$ is sufficiently larger than the threshold $M_{1}^{\beta}+M_{2}^{\beta}$.

So far, without committing to any specific model and merely relying on expected physical behaviour of the wave-functions, we can deduce that mass corrections below the two body threshold are negative. If the state is located above threshold, some corrections can be positive resulting in cancellations. Also, we would expect that if the state is far above the threshold, the coupling to the two meson channel will tend to zero.

As mentioned before, if the decay channel is open for some threshold, i.e. $E \geq$ $M_{1}+M_{2}$, the denominator of Eq. 2.11 will no longer be a strictly negative number 
and will contain a zero at the two body decay momentum

$$
P_{2 B}=\frac{\sqrt{\left(E^{2}-m_{1}^{2}-m_{2}^{2}\right)^{2}-4 m_{1}^{2} m_{2}^{2}}}{2 E}
$$

In order to compute $\Omega(E)$ when the channel is open, we have to make use of the Kramers-Krönig relation ${ }^{1}$ and a change of variables to take into account the zero in the denominator

$$
\begin{aligned}
& \Omega(E)=\mathcal{P} \int_{0}^{\infty} \frac{\mathrm{d} E_{M_{1} M_{2}}^{\beta}}{E-E_{M_{1} M_{2}}^{\beta}}\left(C_{J_{M_{1}}, L_{M_{1}}, S_{M_{1}}}^{J_{J_{2}}, L_{M_{2}}, S_{M_{2}}}\right)^{2} \frac{P E_{M_{1}}^{\beta} E_{M_{2}}^{\beta}}{E_{M_{1} M_{2}}^{\beta}} \mathcal{I}^{2}\left(P, m_{1}, m_{2}, m_{3}, m_{4}\right) \\
& +\left.\pi i \frac{P E_{M_{1}}^{\beta} E_{M_{2}}^{\beta}}{E_{M_{1} M_{2}}^{\beta}} \mathcal{I}^{2}\left(P, m_{1}, m_{2}, m_{3}, m_{4}\right)\left(C_{J_{M_{1}}, L_{M_{1}}, S_{M_{1}}}^{J_{\phi}, L_{\phi}, S_{\phi}}\right)_{M_{2}, L_{M_{2}}, S_{M_{2}}}\right|_{E_{M_{1} M_{2}}^{\beta}=E} .
\end{aligned}
$$

The $\mathcal{P}$ symbol indicates a Cauchy principal value integral [35] and gives the mass shift to the physical state from coupling to the two meson channel $\beta$. The imaginary part of the expression is related to the decay width of $\Psi \rightarrow M_{1}^{\beta}+M_{2}^{\beta}$ process via

$$
\Gamma\left(\Psi \rightarrow M_{1}+M_{2}\right)=2 \operatorname{Im}\left\{\Omega\left(M_{\Psi}\right)\right\}
$$

which matches the standard decay width formula in Ackleh et al. [36].

Having discussed the rest of the loop mass correction function in detail, we can now turn our attention to our choice of model for $\mathcal{V}$, the Quark Pair Creation Model and look at its matrix element.

\footnotetext{
${ }^{1}$ Named Sokhotski-Plemelj theorem in Mathematics [34].
} 


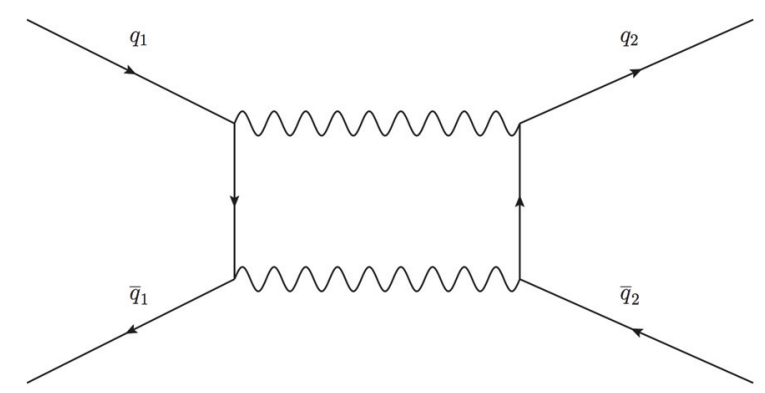

Figure 2.4: An example of a OZI violating process where the quarks of the initial meson, $\mathrm{q}_{1} \overline{\mathrm{q}_{1}}$ annihilate to create a new pair of $\mathrm{q}_{2} \overline{\mathrm{q}}_{2}$.

\subsection{The OZI Rule and the Quark Pair Creation Model}

The type of strong decays that we consider in this work, and illustrated in Fig. 2.1 is known as the OZI-allowed decay. The OZI rule (named after Okubo, Zweig and Iizuka $[37,38])$ states that diagrams like the one presented in Fig. 2.4 are suppressed relative to a decay mechanism like the one presented in Fig. 2.1. It is believed [21] that this phenomenon is related to the running of the strong coupling constant, $\alpha_{s}$. Gluons created in a process like that of Fig. 2.4 are high energy as they have to create the new hadrons to which the parent state is to decay. From our discussion of chapter 1 we know that at high energies, gluons couple weakly due to asymptotic freedom. Similarly, in a decay process like Fig. 2.1, the process is low energy and as such cannot be understood by perturbation theory and we must use non-perturbative models.

The first effort on modeling OZI-allowed strong decays was made by Micu [39]. There she proposed that the quark anti-quark pair (as seen in Fig.2.1) were created with vacuum quantum numbers $0^{++}$or in spectroscopic notation, ${ }^{3} P_{0}$. She then proceeded to calculate decay rates for a few light mesons by taking the spatial part of the matrix element as a free parameter and computing only spin, angular momentum 
and flavour couplings that arose in the calculation. Taking the spatial part to be a free parameter meant that no assumption was made on the binding potential or the momentum space distribution of the created $q \bar{q}[39]$.

Later, the Orsay group further developed the ${ }^{3} P_{0}$ model [40], now called the Quark Pair Creation Model, by writing out a transition matrix which included explicit meson wave functions and overlap integrals in momentum space. They used 3D isotropic harmonic oscillator wave functions and thus were able to calculate, analytically, the overlap integrals. This improved Micu's model as overlap integrals were no longer free parameters.

In their work, Le Yaouanc et. al. (the Orsay group) used a property of Elementary Meson Emission Models (EME) called "additivity" [41]. In these models, even though the meson is a composite object made of quarks and anti-quarks (as discussed in chapter 1), their emission and absorption is described as that of an elementary particle ( Yukawa's Pion emission from a $q$ or $\bar{q}$ is the prime example of this [42]). The additivity property in EME is the idea [40, 43, 44] that some hadronic properties are the sum of the contributions by one individual constituent quark at a time, with all others being spectators.

This idea was generalized by the Orsay group to the following postulate:

"In a given process, a minimum number of quarks are active while the rest remain spectators."

In a hadronic decay in the context of the QPC model, this means all original quarks are spectators and the $q \bar{q}$ pair is created from the vacuum hence the quantum numbers specified before. Such a postulate simplifies the calculation tremendously as it means the created pair has to be in a singlet state in colour and flavour with zero net momentum and zero electric charge. 
As we intend to calculate the matrix element for $A \rightarrow B+C$, for clarity of notation in this section we use $\phi \rightarrow A, M_{1} \rightarrow B$ and $M_{2} \rightarrow C$ for the labels of our mesons. So $\mathrm{A}$ is the parent with $\mathrm{B}$ and $\mathrm{C}$ her daughters. We begin by writing the S-Matrix of the interaction $[40]$

$$
\hat{S}=\mathbf{1}-2 \pi i \delta\left(E_{f}-E_{i}\right) \hat{T}
$$

with $\hat{T}$ given by $[45,46]$

$$
\begin{array}{r}
\hat{T}=-3 \gamma \sum_{m}\langle 11 m-m \mid 00\rangle \sqrt{96 \pi} \int \mathrm{d}^{3} p_{q} \mathrm{~d}^{3} p_{\bar{q}} \delta^{3}\left(\overrightarrow{\boldsymbol{p}}_{q}+\overrightarrow{\boldsymbol{p}}_{\bar{q}}\right) \mathcal{Y}_{1 m}\left(\frac{\overrightarrow{\boldsymbol{p}}_{q}-\overrightarrow{\boldsymbol{p}}_{\bar{q}}}{2}\right) \times \\
\chi_{1-m} \phi_{0} \omega_{0} b_{q}^{\dagger}\left(\overrightarrow{\boldsymbol{p}}_{q}\right) d_{\bar{q}}^{\dagger}\left(\overrightarrow{\boldsymbol{p}}_{\bar{q}}\right)
\end{array}
$$

where $\chi_{1-m}$ is the spin wave function, $\mathcal{Y}_{1 m}$ is the solid harmonic (defined in terms of Spherical Harmonics: $\left.\mathcal{Y}_{L, M}(\overrightarrow{\boldsymbol{r}}) \equiv|\overrightarrow{\boldsymbol{r}}|^{L} Y_{L, M}(\hat{\mathbf{r}})\right)$ describing the momentum space distribution of the created pair, $\omega_{0}$ describes the colour wave function which has to be a colour singlet and $\phi_{0}$ is the flavour wave function of the created pair which in this work we take to be an $S U$ (3) flavour singlet: $\phi_{0}=\frac{1}{\sqrt{3}}(u \bar{u}+d \bar{d}+s \bar{s})$. The factor of 3 is there for convenience: it cancels the colour factor of $1 / 3$ arising from the colour wave function overlap calculation (see Appendix A). $\sqrt{96 \pi}$ comes from the field theory conventions and normalizations (see Appendix B). $b^{\dagger}$ and $d^{\dagger}$ are creation operators for the quark and the anti-quark, respectively. Finally $\gamma$ is the only free parameter in this model and it sets the strength of the vertex (the black circle in Fig. $2.1)$.

An equivalent way of formulating Eq. 2.16 is to write it as a Hamiltonian involving Dirac fermion fields

$$
H_{I}=g \int \mathrm{d}^{3} x \bar{\psi} \psi
$$

where $g$ can be related to $\gamma$ with $\gamma=\frac{g}{2 m_{q}}, m_{q}$ being the mass of the quark in $q \bar{q}$ 
created pair [36]. Since the probability to generate heavier quarks is suppressed and the interaction vertex should not favour their creation, in our calculations we will use $\gamma_{\text {eff }}=\frac{m_{n}}{m_{q}} \gamma_{0}$ where $m_{q}$ is the mass of the created quark pair and $m_{n}$ is $m_{u}=m_{d}[32$, 47]. The QPC model is the non relativistic reduction of Eq. 2.18.

Since the introduction of this model, studies of it have focused mainly on numerical predictions and calculations of strong decay widths (and more recently mass shifts). Some studies have included some small modification by adding an extra spatial dependence which in effect takes into account the finite size of the created constituent quarks and adds an extra parameter, the effective quark radius, $r_{q}$. The form of this modification is usually taken to be $e^{-\frac{2 r_{q}^{2} p^{2}}{3}}[48-52]$. The rest of the equation as well as the general structure of the pair production mechanism has not been modified.

With an explicit form for $\hat{T}$ at hand, we can proceed to write down the matrix element, $\langle B C|\hat{T}| A\rangle$. To do so, we must first define the mock meson wave function used in the calculation[46]:

$$
\begin{aligned}
& \left|A\left(n_{A}^{2 S_{A}+1} L_{A_{J_{A}, M_{J}}}\right)\left(\overrightarrow{\boldsymbol{P}}_{A}\right)\right\rangle=\sum_{M_{L_{A}}, M_{S_{A}}}\left\langle L_{A} M_{L_{A}} S_{A} M_{S_{A}} \mid J_{A} M_{J_{A}}\right\rangle \times \\
& \quad \int \mathrm{d}^{3} \overrightarrow{\boldsymbol{p}}_{A} \chi_{S_{A} M_{S_{A}} \bar{q}} \phi_{A}^{q \bar{q}} \omega_{A}^{q \bar{q}}\left|q\left(\left(\frac{m_{q}}{m_{q}+m_{\bar{q}}}\right) \overrightarrow{\boldsymbol{P}}_{A}+\overrightarrow{\boldsymbol{p}}_{A}\right) \bar{q}\left(\left(\frac{m_{\bar{q}}}{m_{q}+m_{\bar{q}}}\right) \overrightarrow{\boldsymbol{P}}_{A}-\overrightarrow{\boldsymbol{p}}_{A}\right)\right\rangle
\end{aligned}
$$

which is normalized to

$$
\left\langle A\left(n_{A}^{2 S_{A}+1} L_{A_{J_{A}, M_{J_{A}}}}\right)\left(\overrightarrow{\boldsymbol{P}}^{\prime}\right) \mid A\left(n_{A}^{2 S_{A}+1} L_{A_{J_{A}, M_{J_{A}}}}\right)(\overrightarrow{\boldsymbol{P}})\right\rangle=\delta^{3}\left(\overrightarrow{\boldsymbol{P}}-\overrightarrow{\boldsymbol{P}}^{\prime}\right)
$$

$\overrightarrow{\boldsymbol{P}}_{A}$ is the momentum of meson A, $\overrightarrow{\boldsymbol{p}}_{A}$ is the momentum of its constituents and $\chi, \phi$ and $\omega$ are wave functions for combining spins, flavour and colour of the constituent quarks to get the corresponding properties of the meson. Having discussed the other quantum numbers before, we just note that $n_{A}$ is the radial quantum number related to the number of nodes in the radial part of the meson wave function $\left(\#\right.$ nodes $\left.=n_{A}-1\right)$. 


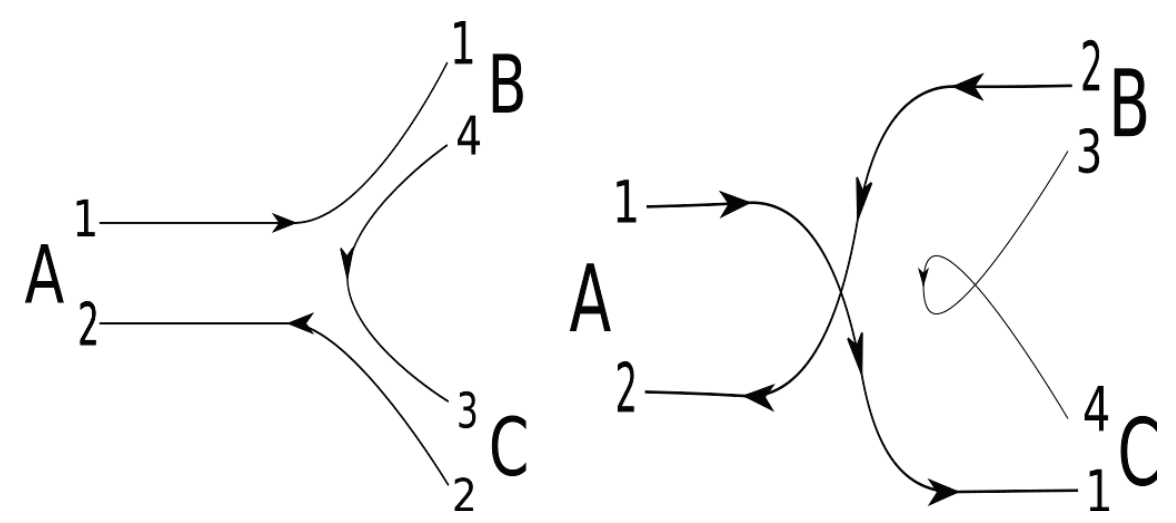

Figure 2.5: The two possible arrangements for a meson decay in the Quark Pair Creation Model: on the left the initial quark ends in meson B while on the right it ends in meson $\mathrm{C}$. In practice one of these two diagrams is often zero.

We can now sandwich the operator $\hat{T}$ between three mock meson wave functions to obtain the explicit matrix element form of the Quark Pair Creation Model in the

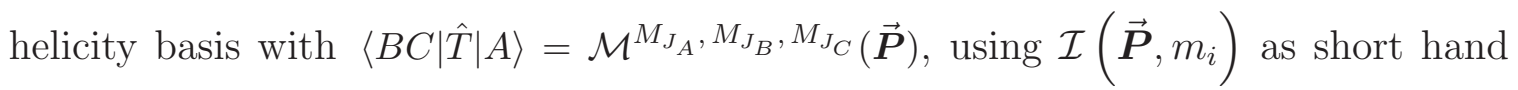
for $\mathcal{I}\left(\overrightarrow{\boldsymbol{P}}, m_{1}, m_{2}, m_{3}, m_{4}\right)$

$$
\begin{gathered}
\mathcal{M}^{M_{J_{A}}, M_{J_{B}}, M_{J_{C}}}(\overrightarrow{\boldsymbol{P}})=\gamma \sum\left\langle L_{A} M_{L_{A}} S_{A} M_{S_{A}} \mid J_{A}, M_{J_{A}}\right\rangle\left\langle L_{B} M_{L_{B}} S_{B} M_{S_{B}} \mid J_{B}, M_{J_{B}}\right\rangle \\
\left\langle L_{C} M_{L_{C}} S_{C} M_{S_{C}} \mid J_{C}, M_{J_{C}}\right\rangle\langle 1 m 1-m \mid 00\rangle\left\langle\chi_{S_{B} M_{S_{B}}}^{14} \chi_{S_{C} M_{S_{C}}}^{32} \mid \chi_{S_{A} M_{S_{A}}}^{12} \chi_{1-m}^{34}\right\rangle \\
{\left[\left\langle\phi_{B}^{14} \psi_{C}^{32} \mid \phi_{A}^{12} \phi_{0}^{34}\right\rangle \mathcal{I}\left(\overrightarrow{\boldsymbol{P}}, m_{i}\right)+(-1)^{1+S_{A}+S_{B}+S_{C}}\left\langle\phi_{B}^{32} \psi_{C}^{14} \mid \phi_{A}^{12} \phi_{0}^{34}\right\rangle \mathcal{I}\left(-\overrightarrow{\boldsymbol{P}}, m_{i}\right)\right]}
\end{gathered}
$$

where quark and meson labels correspond to that of Fig. 2.5. The sum runs over the possible values of $M_{L_{A}}, M_{S_{A}}, m, M_{L_{B}}, M_{S_{B}}, M_{L_{C}}$ and $M_{S_{C}}$. Eq. 2.21 is in the helicity basis. However, we rewrite the matrix element in terms of partial waves, $\mathcal{M}^{L, S}$, which are also what experiments measure. The conversion is done in Ref. [46] via the Jacob-Wick formula $[53,54]$ after specializing to the center of mass of the 
parent meson A where $\overrightarrow{\boldsymbol{P}}_{A}=0$. Thus we arrive at

$$
\begin{array}{r}
\mathcal{M}^{L S}=\frac{\sqrt{4 \pi(2 S+1)}}{2 J_{A}+1} \sum_{M_{J_{B}}, M_{J_{C}}}\left\langle L 0 S M_{J_{A}} \mid J_{A} M_{J_{A}}\right\rangle\left\langle J_{B} M_{J_{B}} J_{C} M_{J_{C}} \mid S M_{J_{A}}\right\rangle \times \\
\left.\mathcal{M}^{M_{J_{A}}, M_{J_{B}}, M_{J_{C}}(P \hat{\mathbf{z}})}\right|_{M_{J_{A}}=M_{J_{B}}+M_{J_{C}}}
\end{array}
$$

where $P$ is the magnitude of vector $\overrightarrow{\boldsymbol{P}}=\overrightarrow{\boldsymbol{P}}_{B}=-\overrightarrow{\boldsymbol{P}}_{C}$, the two body decay momentum. The valid partial waves denoted by $L$ and $S$ are given by $\left|J_{B}-J_{C}\right| \leq S \leq J_{B}+J_{C}$ and $\left|J_{A}-S\right| \leq L \leq J_{A}+S$.

$\mathcal{I}\left(P, m_{i}\right)$ given in Eq. 2.21 (also repeated implicitly in Eq. 2.22) is the overlap integral which given explicitly in terms of the various meson wave functions is written as

$$
\begin{aligned}
\mathcal{I}\left(\overrightarrow{\boldsymbol{P}}, m_{i}\right)= & \sqrt{96 \pi} \int \mathrm{d}^{3} p \mathcal{Y}_{1 m}(\overrightarrow{\boldsymbol{p}}) \psi_{n_{A}, L_{A}, M_{L_{A}}}(\overrightarrow{\boldsymbol{p}}+\overrightarrow{\boldsymbol{P}}) \times \\
& \psi_{n_{B}, L_{B}, M_{L_{B}}}^{*}\left(\overrightarrow{\boldsymbol{p}}+\frac{m_{4}}{m_{1}+m_{4}} \overrightarrow{\boldsymbol{P}}\right) \psi_{n_{C}, L_{C}, M_{L_{C}}}^{*}\left(\overrightarrow{\boldsymbol{p}}+\frac{m_{3}}{m_{2}+m_{3}} \overrightarrow{\boldsymbol{P}}\right) .
\end{aligned}
$$

The wave functions $\psi_{A}, \psi_{B}$ and $\psi_{C}$ are approximated by $3 \mathrm{D}$ isotropic harmonic oscillator wave functions written (in momentum space) as

$$
\begin{aligned}
& \psi_{n, L, M_{L}}^{S H O}(\overrightarrow{\boldsymbol{p}})=R_{n L}^{S H O}(|\overrightarrow{\boldsymbol{p}}|) Y_{L, M_{L}}\left(\theta_{p}, \phi_{p}\right) \\
& R_{n L}^{S H O}(|\overrightarrow{\boldsymbol{p}}|)=\frac{(i)^{L}(-1)^{n-1}}{\beta^{3 / 2}} \sqrt{\frac{2(n-1) !}{\Gamma\left(n+L+\frac{1}{2}\right)}} L_{n-1}^{L+\frac{1}{2}}\left(p^{2} / \beta^{2}\right) e^{-p^{2} /\left(2 \beta^{2}\right)}
\end{aligned}
$$

where $L_{n-1}^{L+1 / 2}$ is an associated Laguerre polynomial [55]. $\beta$ is the harmonic oscillator parameter and we will study its effects in a later chapter.

Detailed studies have been done to calculate Eq. 2.22, with the most computationally intensive part being the overlap integral. The calculations are tedious and 
the derived formulas are complicated $[46,56]$. In our work, we have automated the computation using Mathematica [57]. The technique that we use to simplify and solve for the matrix element was proposed by Ref. [56] and it recognizes that

- We are free to choose the $\hat{\mathbf{z}}$ direction for $\overrightarrow{\boldsymbol{P}}$ as made explicit in Eq. 2.22.

- There exists an exponential term in the overlap integral which originates from the three wave functions (and perhaps the quark form factor, if it is included in the model)

What was suggested by Roberts et al. [56] was to apply the change of variable $\overrightarrow{\boldsymbol{p}} \rightarrow$ $\overrightarrow{\boldsymbol{p}}^{\prime}-x \overrightarrow{\boldsymbol{P}}$ and solve for $x$ in the exponent of the exponential such that the $\overrightarrow{\boldsymbol{p}}^{\prime} \cdot \overrightarrow{\boldsymbol{P}}$ term cancels. The rest of the calculation is performed symbolically with Mathematica. We will study the behaviour of the momentum overlap integral in more detail in the next chapter.

Once the integrals are evaluated we will have explicit expressions for $\mathcal{M}^{L S}$. Then $\Omega(E)$ can be calculated for each partial wave $(L, S)$ with the total correction being the sum of the individual contributions. If the aim of the calculation is to obtain physical masses, the process is iterated. To start, the bare mass is used as the input in Eq. 2.10. The physical mass that is calculated is then used as input for Eq. 2.10 and the process is repeated until it converges. In this work, that is not our aim so we run the program only once, taking the bare mass as a good approximation of the physical mass, or in algebraic form

$$
M_{\text {Phys }}=m_{0}+\Omega\left(m_{0}\right) .
$$




\subsection{Short Note on Other Models of Strong Decay}

In this section we will summarize the work of Ackleh et al. in Ref. [36]. The purpose of this, other than for completeness, is to further motivate and justify the use of the QPC model in our calculations.

In their paper, Ackleh et al. studied strong decays of light mesons using scalar confining interaction and one gluon exchange potentials. The QPC model is a special case of the scalar confining interaction while the Cornell model is related to a Lorentz vector interaction. We will begin the discussion by first briefly summarizing the Cornell Model of strong decay [5] as it was among the first models of strong decay and will be an illuminating case. Other models will be discussed very briefly and the interested reader is encouraged to refer to Ref. [36]. It should be noted that for all these models the general form of Eq. 2.10 and 2.11 are unchanged except for the matrix element given in the numerator of Eq. 2.11.

\subsubsection{Cornell Model of Strong Decay}

In chapter 1 we discussed the naive Cornell Model as formulated in Ref. [5]. However, in that same paper, the Cornell group also proposed a decay model to connect the naive spectrum to the continuum. They postulated that the same mechanism that causes binding in mesons, i.e. the potential of Eq. 1.2, is also responsible for pair creation leading to OZI-allowed strong decays. In their model, they specifically attributed this effect to the linear confining part of the potential, arguing that the colour Coulomb potential is only relevant at small quark separations and, at larger distances where the decay occurs, it is of little to no consequence. It should also be noted in their calculations they ignore spin-orbit $(\overrightarrow{\boldsymbol{S}} \cdot \overrightarrow{\boldsymbol{L}})$ and hyperfine $\left(\overrightarrow{\boldsymbol{S}_{q}} \cdot \overrightarrow{\boldsymbol{S}_{\bar{q}}}\right)$ interactions in the naive spectrum as well as the continuum channels (so only the 
confining potential in Eq. 1.5, and ignoring the other parts).

With that understanding, they proposed the following interaction Hamiltonian

$$
H_{I}=\frac{1}{2} \sum_{a=1}^{8} \int \mathrm{d}^{3} x \mathrm{~d}^{3} y \bar{\psi}(\overrightarrow{\boldsymbol{x}}) \gamma_{0} \frac{\lambda_{a}}{2} \psi(\overrightarrow{\boldsymbol{x}}) V_{0}(\overrightarrow{\boldsymbol{x}}-\overrightarrow{\boldsymbol{y}}) \bar{\psi}(\overrightarrow{\boldsymbol{y}}) \gamma_{0} \frac{\lambda_{a}}{2} \psi(\overrightarrow{\boldsymbol{y}})
$$

where $V_{0}=\frac{3}{4} V$ for $V$ in Eq. 1.2, $\gamma_{0}$ is a Dirac matrix and the $\lambda_{a}$ are Gell-Mann matrices.

We observe immediately that the interaction in Eq. 2.26 has a different Lorentz structure than that of the QPC model: while QPC assumes a scalar interaction the Cornell Hamiltonian is the time component of a vector interaction. Another difference here is that while we have one spectator quark, the additivity property is not as strong as it was in the QPC model case. Here, the momentum of the parent quark changes and the created quark anti-quark pair receive nonzero momentum.

With this Hamiltonian the Cornell group proceeded to calculate the Charmonium spectrum by incorporating virtual loops and decay widths though they truncated their sum of mesonic loops to include only S-wave $(L=0)$ mesons. In their approach, they would construct a matrix of naive states where Eq. 2.26 would provide self-energy corrections and off-diagonal matrix elements. The physical masses and their widths are then calculated by diagonalizing the matrix.

The most appealing property of this model is the fact that once the parameters of the naive potential in Eq. 1.2 are set, the entire spectrum including the corrections are, in principle, determined and there are no further free parameters. One still has to go through the iterative process if the goal of the calculation is to determine physical masses, but that is universal to all models in this framework. However, the Cornell decay model has not been pursued as a model of strong decays due to the time-like Lorentz vector structure that it assumes. This goes contrary to what is known of 
strong decays via Lattice QCD which indicates the potential as a long range scalar structure, not a vector one [58]. Furthermore, the model gives inaccurate prediction of P-wave multiplet splittings in Charmonium [59].

\subsubsection{Scalar Confining Interaction and One Gluon Exchange Model}

Ackleh et al. in Ref. [36] began their study of other possible mechanisms of strong decays by accepting the assumption made by Eichten et. al. [5] that the interaction causing the decay of a meson is driven by the same potential that binds its quarks. Thus they can generalize the form of Eq. 2.26 and write

$$
H_{I}=\frac{1}{2} \int \mathrm{d}^{3} x \mathrm{~d}^{3} y J^{a}(\overrightarrow{\boldsymbol{x}}) \mathcal{K}(|\overrightarrow{\boldsymbol{x}}-\overrightarrow{\boldsymbol{y}}|) J^{a}(\overrightarrow{\boldsymbol{y}})
$$

where $J^{a}$ are quark-currents given below [36]

$$
J^{a}(\overrightarrow{\boldsymbol{x}})= \begin{cases}\bar{\psi}(\overrightarrow{\boldsymbol{x}}) \frac{\lambda_{a}}{2} \psi(\overrightarrow{\boldsymbol{x}}) & \text { scalar confining interaction } \\ \bar{\psi}(\overrightarrow{\boldsymbol{x}}) \frac{\lambda_{a}}{2} \gamma_{0} \psi(\overrightarrow{\boldsymbol{x}}) & \text { colour Coulomb one gluon exchange } \\ \left(\bar{\psi}(\overrightarrow{\boldsymbol{x}}) \frac{\lambda_{a}}{2} \vec{\gamma} \psi(\overrightarrow{\boldsymbol{x}})\right)_{T} & \text { transverse one gluon exchange }\end{cases}
$$

The kernels in Eq. 2.27 are then written as

$$
\mathcal{K}= \begin{cases}+\frac{3}{4}(b r+C) & \text { linear confining potential } \\ +\frac{\alpha_{s}}{r} & \text { colour Coulomb one gluon exchange } \\ -\frac{\alpha_{s}}{r} & \text { transverse one gluon exchange }\end{cases}
$$


The Cornell decay model is now a special case of Eq. 2.27, where one takes the scalar confining potential of Eq. 2.29 with the time-like vector current of Eq. 2.28. The quark pair creation model on the other hand is only the constant term in the scalar confining interaction kernel with the scalar current. The general structure of matrix elements is still the same as that of the ${ }^{3} P_{0}$ model as it can be factorized into angular momentum, spin, colour and flavour re-coupling coefficients and overlap integrals of the three wave functions involved.

The calculations are then performed using simple harmonic oscillator wave functions while setting the HO parameter of all three wave functions to a shared value of $\beta=0.4 \mathrm{GeV}$. This is a reasonable approximation as Ackleh et al. concern themselves exclusively with calculating/comparing decay widths in the light quark sector and the constituent masses of up, down and strange quarks are nearly equal.

In their rather comprehensive study they find that the linear confining interaction as a Lorentz scalar and the QPC give similar results provided that the $\gamma$ parameter in Eq. 2.17, which sets the strength of that vertex and the $b$ parameter in the linear potential of Eq. 2.29 are taken as free parameters. However, this is a problem as $b$ is the string tension which has a preferred value in meson spectroscopy $\left(b \approx 0.18 \mathrm{GeV}^{2}\right.$ $[5,60])$ which then results in this potential over-estimating the decay rates. They also find that the one gluon exchange potentials are numerically negligible contributions in all channels except for ${ }^{3} P_{0} \rightarrow{ }^{1} S_{0}+{ }^{1} S_{0}$.

These models are, however, extremely computationally intensive even with the assumptions made by Ackleh et al., with matrix elements involving confluent hypergeometric functions. This is not a major impediment for calculating widths. However, for our purposes of calculating loops which require integrating over matrix elementsquared it becomes a level of complexity that cannot be justified given that a simpler 
model, the QPC model, produces results which are in better agreement with experiment. Of course, in the QPC model there were still assumptions made that can be relaxed given advancements in computing. It is also worth stating that the study in Ref. [36] of various models of the strong decays of mesons has not been replicated for charmonium, bottomonium or flavoured systems (such as $c \bar{s}$ or $b \bar{c}$ and so on) which could shed more light on the study of strong decays than the light quark sector. 


\section{Chapter 3}

\section{Detailed Study of the Overlap Integrals}

The Quark Pair Creation model has become the "go-to" model for meson loop calcula-

tions $[24,26,27,32,49,50,61-63]$. In such calculations, there are always assumptions made on what the choice of parameters of the model are and how they are to be determined. Of course the ${ }^{3} P_{0}$ model is itself an approximation of the strong pair creation vertex but most authors go even further within the model. Almost all researchers (except for Ref. [47], to our knowledge) take harmonic oscillator wave functions as previous research has shown them to be reliable estimates of the exact numerical solutions of the potential model [46].

In this chapter we seek to understand the sensitivity of the coupled channel model with the QPC decay vertex to parameters of the model as well as inclusion of mesons with higher radial and angular quantum numbers in the loop. First we will start with the determination of parameters.

\subsection{Parameter Determination}

Calculations of loops with the QPC model traditionally, regardless of the approximations made, contain at least 2 parameters 
1. $\beta$, the Harmonic Oscillator parameter

2. $\gamma_{0}$, the strength of the strong pair production vertex.

In our study we add a third parameter, $r_{q}$, which is introduced in a Gaussian form to smear the interaction vertex. It was proposed by Ref. [51] and previously discussed in chapter 2. This parameter accounts for the finite size of the created constituent quarks and as we shall see, it plays a crucial role in regulating mass corrections within the QPC model.

In general, the parameters used in these calculations can be divided into two categories

- parameters set before the coupled channel calculation including the inter-quark potential parameters,$V_{q \bar{q}}(r)$, constituent quark masses and harmonic oscillator wave function parameter $\beta$

- parameters set for the coupled channel calculation (only two of them in our case): $\gamma_{0}, r_{q}$.

Fixing the wave function parameter $\beta$ and the constituent masses is connected to what was discussed in chapter 1 . As mentioned there, the parameters of the model are fitted to some low lying states in the spectrum of interest. This procedure determines the constituent masses, string tension parameter, the relativistic smearing and various spin interaction parameters (if one is using the Godfrey-Isgur Quark Model or a similar model).

After solving the Schrödinger equation and acquiring the numerical eigenfunctions and eigenvalues, one can find the $\beta$ that would best describe the true wave function in two ways: 
1. calculate the r.m.s (root mean squared) radius of the exact numerical solution and set equal to the r.m.s radius of the equivalent harmonic oscillator wave function

2. calculate an overlap integral for the harmonic oscillator wave function and the exact numerical solution, fitting for the $\beta$ that maximizes the overlap.

$\beta$ values used in this work are acquired via the former method from the Godfrey-Isgur model and are taken as given inputs along with the "bare" meson masses. We do not repeat the calculations and encourage the interested reader to refer to the original article in Ref. [7] for details, if deemed necessary.

Many calculations of hadron loops have taken the SHO parameter of the three wave functions in Eq. 2.23 to be equal. This approximation was originally made in calculations of decays using this model and later used in calculations of mass corrections. In studies of the light quark sector (bound states of $\mathrm{u}, \mathrm{d}$ and $\mathrm{s}$ quarks), $\beta_{\text {eff }}$ is usually set to $0.4 \mathrm{GeV}[36,64]$. Given that in the limit of perfect symmetry between these quarks we can view them as degenerate in mass this seems a reasonable approximation. In chapter 5, we take this value for the light mesons as well as our "equal $\beta$ " studies of the charm-strange sector. For single- $\beta$ calculations of bottomonium in chapter 4 , we will use $\beta_{\text {eff }}=0.5 \mathrm{GeV}$ which seems to be the preferred choice for bottomonium in the literature [30, 49, 50, 61].

The parameter for the interaction strength, $\gamma_{0}$, and the constituent quark size parameter, $r_{q}$, are fitted to the strong decay width of mesons in the spectrum of interest. For this fit, the matrix elements are calculated within the QPC model using $\beta$ values (be it $\beta_{\text {eff }}$ or un-equal $\beta$ 's) with the expressions then used along with experimental decay widths and their ratios to find a fit for $r_{q}$ and $\gamma_{0}$. The numerical value of $\gamma_{0}$ quoted in various papers depends, of course, on the normalization used for 
Table 3.1: QPC model parameters used in this work. Except for $\gamma_{0}$ which is a unit-less number, all other values are in GeV. Charm-Strange parameters are from Ref. [65], bottomonium from [49] and charmonium parameters from [50]. The charmonium column will be used in future work and is only included here for completeness.

\begin{tabular}{cccc}
\hline Parameter & Charm-Strange & Charmonium & Bottomonium \\
\hline$\gamma_{0}$ & 0.4 & 0.51 & 0.732 \\
$\beta_{\mathrm{eff}}$ & 0.4 & 0.5 & 0.5 \\
$m_{n}=m_{u}, m_{d}$ & 0.22 & 0.33 & 0.33 \\
$m_{s}$ & 0.419 & 0.55 & 0.55 \\
$m_{c}$ & 1.628 & 1.50 & 1.50 \\
$m_{b}$ & - & - & 4.70 \\
\hline
\end{tabular}

the mock meson wave functions. In this work we do not perform fits for parameters and instead use previously published and accepted values given in table 3.1.

We are now in a position to look at equation Eq. 2.11 and study the numerator.

\subsection{General Structure of the Momentum Overlap Integral}

In chapter 2 we discussed the general properties of the mass correction function, $\Omega(E)$ of Eq. 2.10. Most of our discussion centered around the overall sign of the function and made sensible assumptions regarding the behaviour of the matrix element in the numerator. Here, we shall look at some explicit examples of matrix elements for a naive state coupling to continuum intermediate levels. In this section, we will use values for masses, wave function parameters or model parameters that will resemble a bottomonium or charmonium system although making physical calculations is not our goal. In our study of matrix elements, we wish to examine two issues: 
1. Comparison of various matrix elements for excited mesons would help illustrate the convergence of the sum of their contributions in the loop and allow us to determine if approximations made in many previous studies are valid.

2. Study the effect of the quark form factor $\left(r_{q}\right.$, the effective constituent quark radius) on the shape of the matrix element and the sum of total meson-loop contributions.

Given the many possible different combinations of parameters, we begin with the simplest case possible: equal $\beta$ for all three wave functions and equal masses for the constituent quarks involved in Eq. 2.23.

\subsubsection{Single $\beta$ and a form factor}

We start in figures 3.1 and 3.2 with an initial $1 S$ state (i.e. $n=0, L=0$ ) coupling to intermediate states $n S+n S$ (for $n \in(1,2,3,4,5))$ as well as $1 S+n P($ for $n \in(1,2,3)$ ). In both figures, plotted with different values for $r_{q}$, we see a clear decrease in the magnitude of the integral as the radial quantum number $(n)$ in the loop increases. Given that the re-coupling coefficients in Eq. 2.12 are independent of the radial quantum number, we can conclude that higher order terms in $n$, contribute less to the overall correction sum. Another point to raise on this figure is the shape-change due to the inclusion of the quark form factor. Predictably, the form factor reduces the contribution of higher momenta to the loop. This is an important point as the traditional QPC model creates hard vertices [66] and higher momenta contribute more to the loop. To illustrate this better, consider Fig. 3.3 which compares $1 S \rightarrow 3 S+3 S$ and $1 S \rightarrow 3 S+3 P$ for different values of $r_{q}$. 


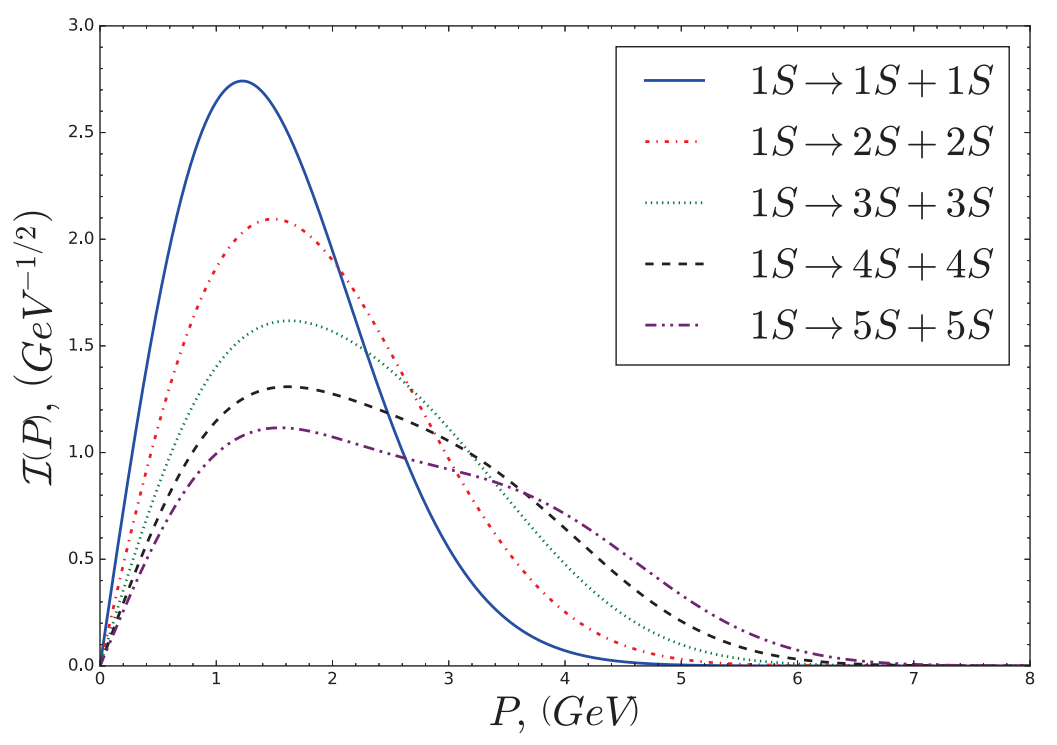

(a) $r_{q}=0.0 \mathrm{fm}$

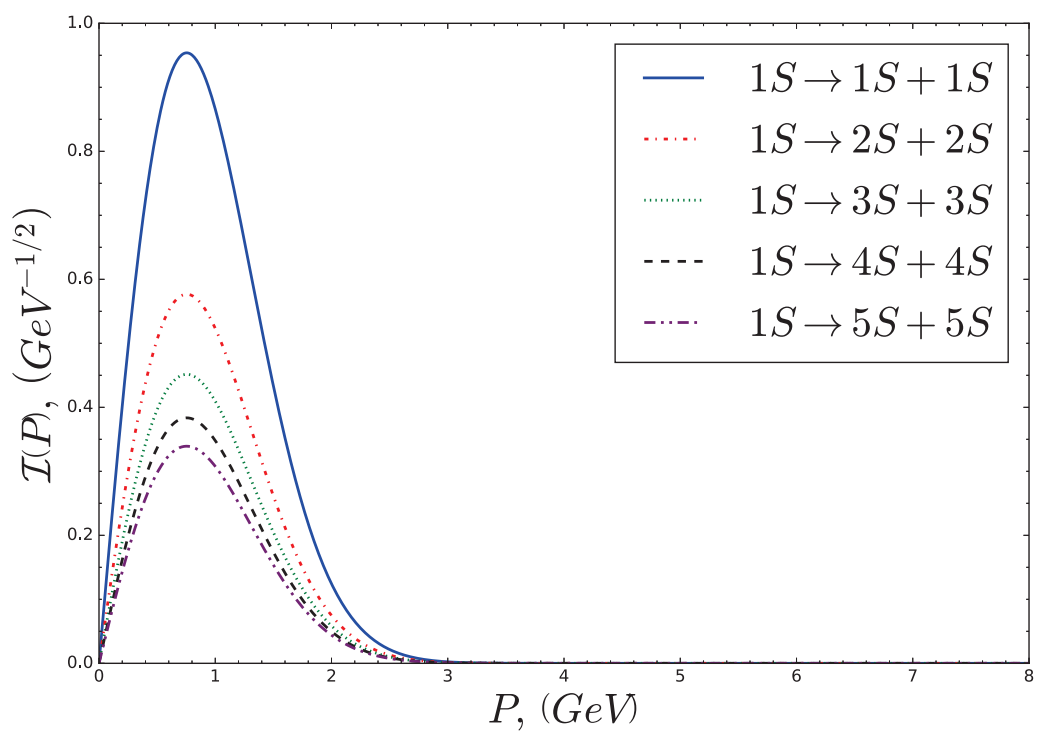

(b) $r_{q}=0.3 \mathrm{fm}$

Figure 3.1: Momentum overlap integral of the matrix element $\langle n S+n S|\hat{T}| 1 S\rangle$ for $n=1-5$. In both plots, $\beta=0.5 \mathrm{GeV}$ is the single harmonic oscillator parameter used for all wave functions. Constituent masses are taken to be equal. $P$ is the centre of mass momentum of the "out-going" mesons. 


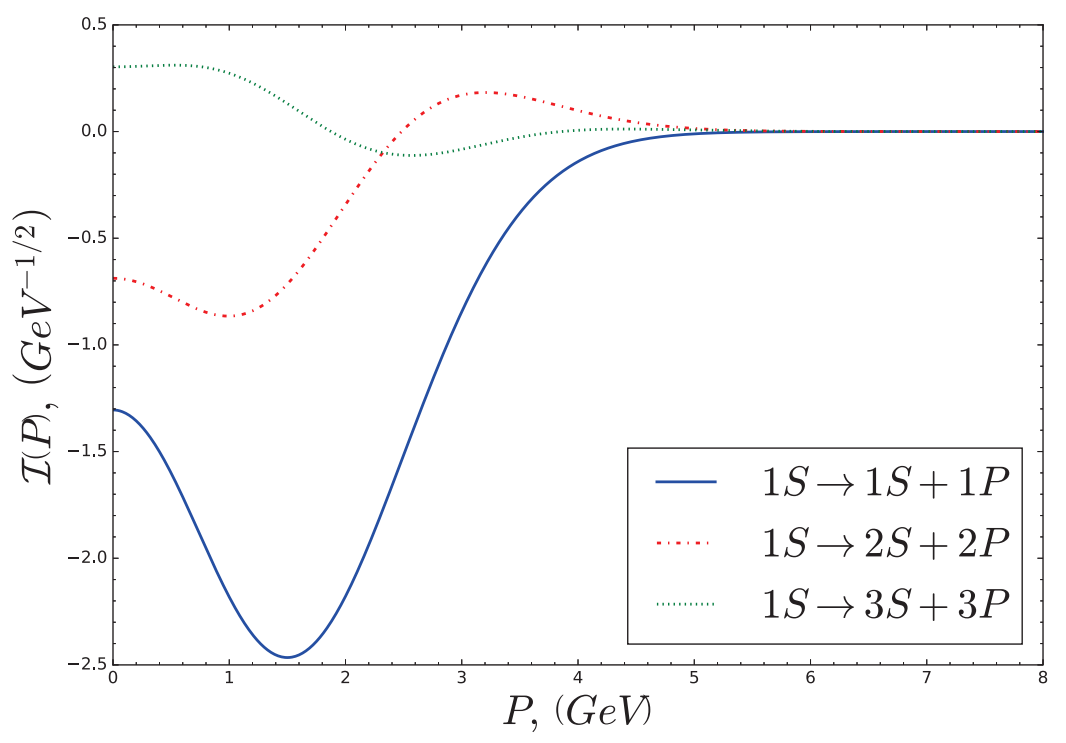

(a) $r_{q}=0.0 \mathrm{fm}$

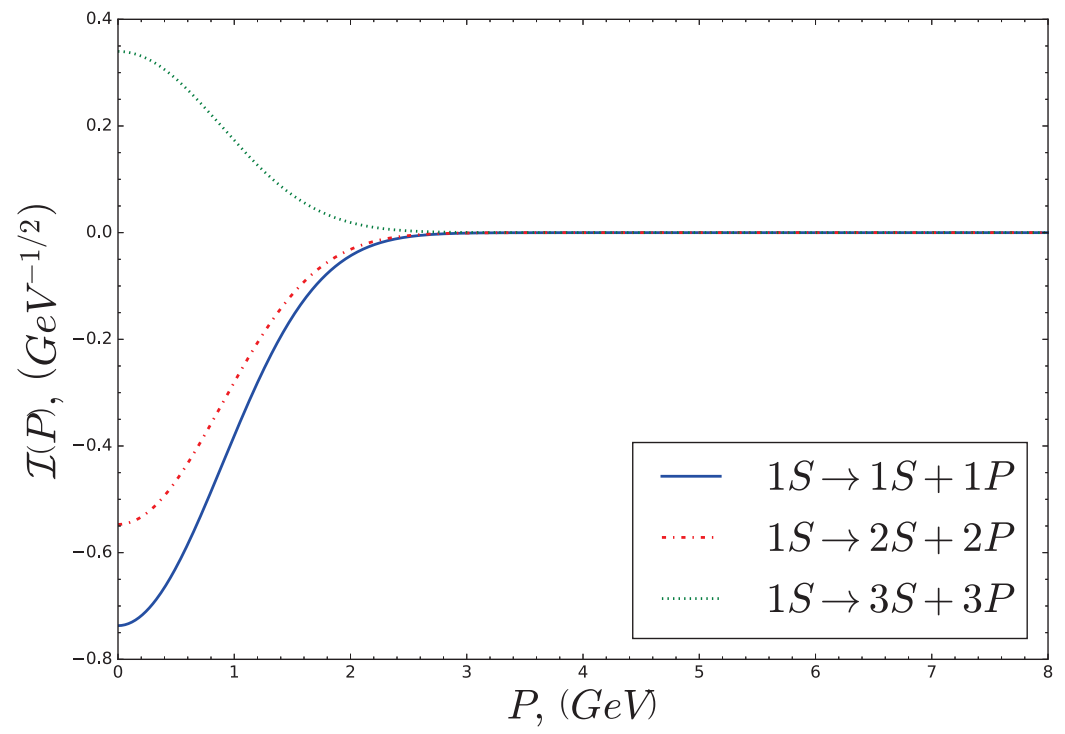

(b) $r_{q}=0.3 \mathrm{fm}$

Figure 3.2: Momentum overlap integral of the matrix element $\langle n S+n P|\hat{T}| 1 S\rangle$ for $n=1-3$. In both plots, $\beta=0.5 \mathrm{GeV}$ is the simple harmonic oscillator parameter used for all wave functions. Constituent masses are taken to be equal. $P$ is the centre of mass momentum of the "out-going" mesons. 


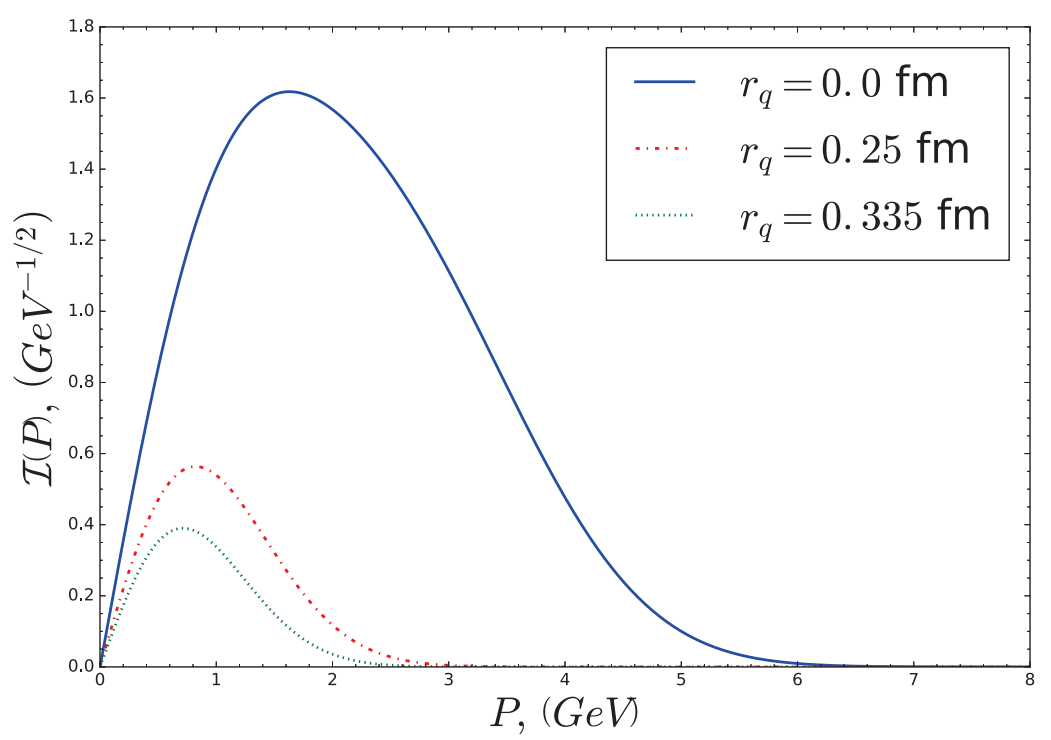

(a) $1 S \rightarrow 3 S+3 S$

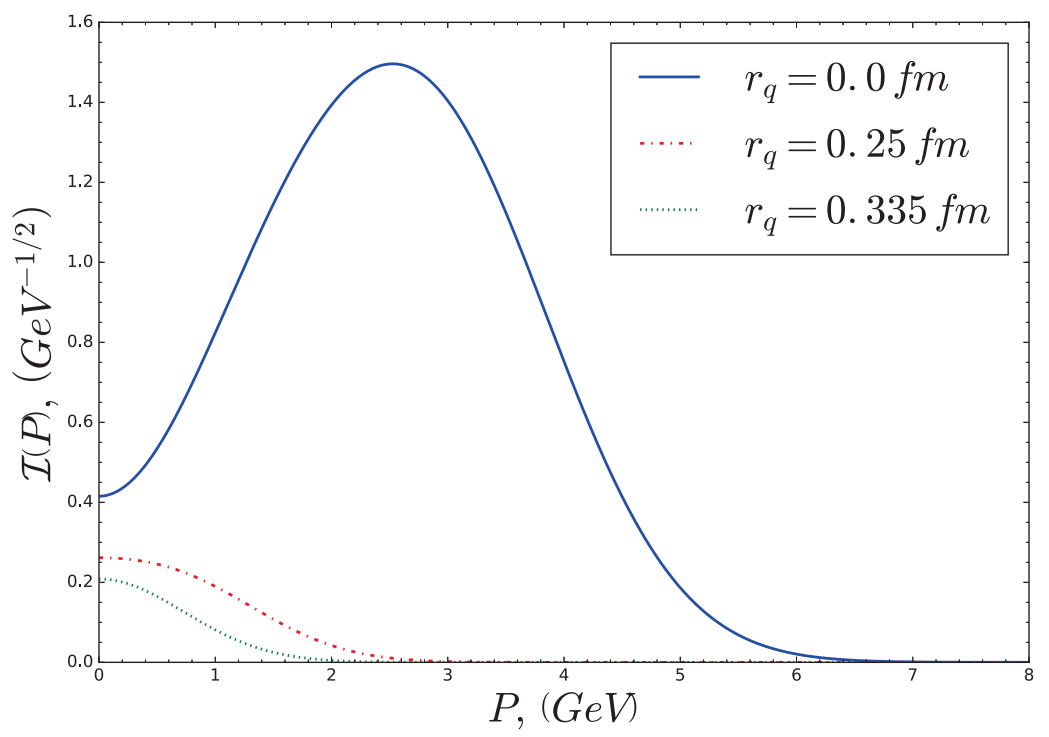

(b) $1 S \rightarrow 3 S+3 P$

Figure 3.3: Effect of the quark form factor on the momentum overlap integral. $1 S \rightarrow 3 S+3 S$ and $1 S \rightarrow 3 S+3 P$ are used as test cases. The $r_{q}=0.25,0.335 \mathrm{fm}$ are at the bounds of accepted values for $r_{q}$ suggested by Refs. [50, 51]. The effect of the form factor on higher momenta is clearly demonstrated by the dampening of $\mathcal{I}(P)$ in that regime. 


\subsubsection{Unequal $\beta$ and unequal mass}

In the previous section we made the approximation of setting the masses of the quarks to be equal as well as using an effective $\beta$ for all the wave functions. These are the assumptions that we will relax in this section in order to study the momentum overlap integral. The assumption on wave function parameters are expected to be important as different $\beta$ values set the size of the wave function and, by extension, the size of the meson. Hadron spectroscopy is perhaps unique in physics where a state, small in size, can decay to two bodies that are larger [5]. We again turn our attention to an initial S-wave, coupling to the same final states as before.

In Fig. 3.4 we look at two matrix element test cases which we also considered in

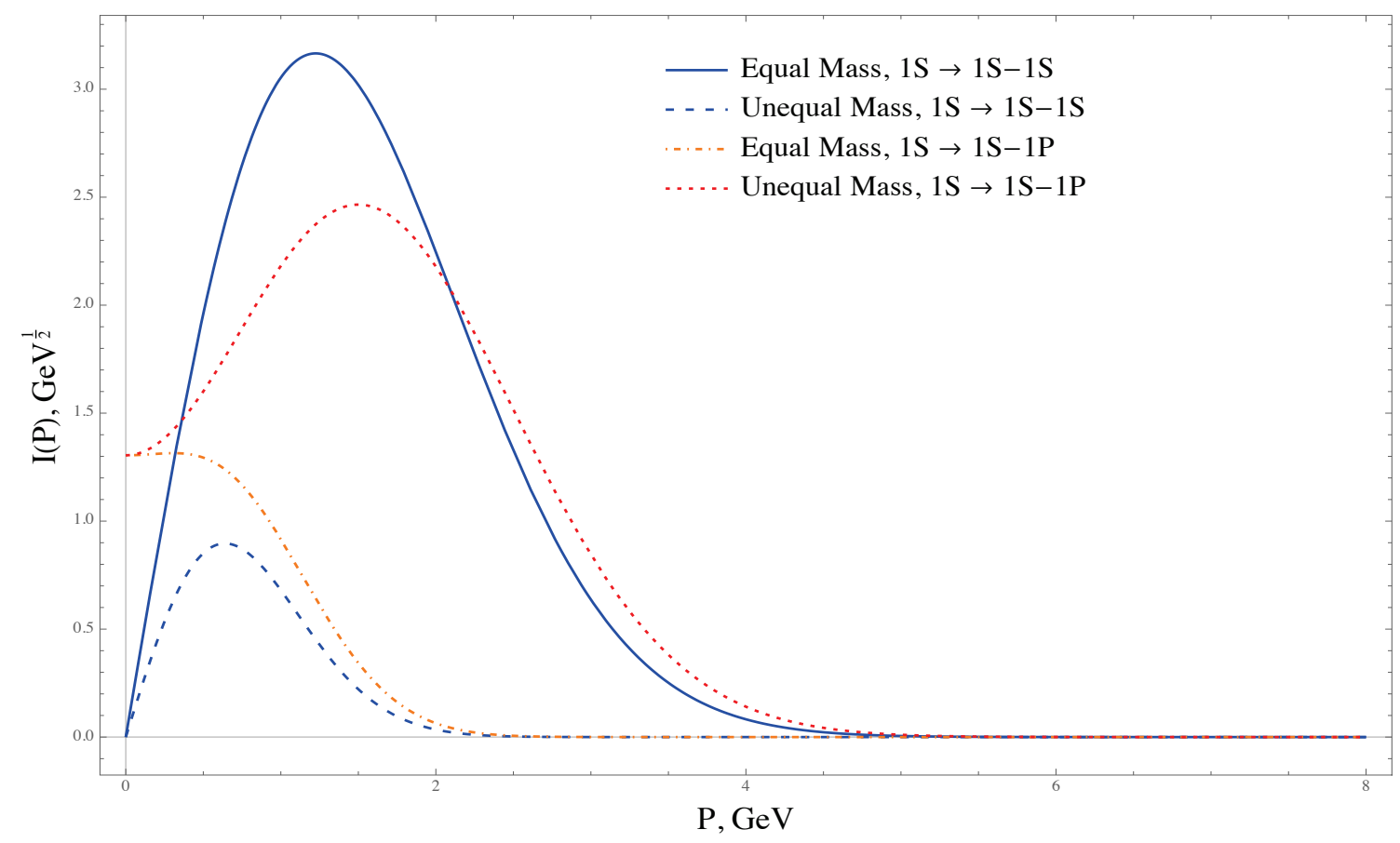

Figure 3.4: Effect of the constituent quark mass on two sample matrix elements: $\langle 1 S+1 S|T| 1 S\rangle$ and $\langle 1 S+1 P|T| 1 S\rangle . r_{q}$ is set to 0 and $\beta_{\text {eff }}=0.5 \mathrm{GeV}$. Constituent masses used are that of b-quark for the heavy parent meson and u-quark for the created pair. Mass values taken from the fourth column of table 3.1. 


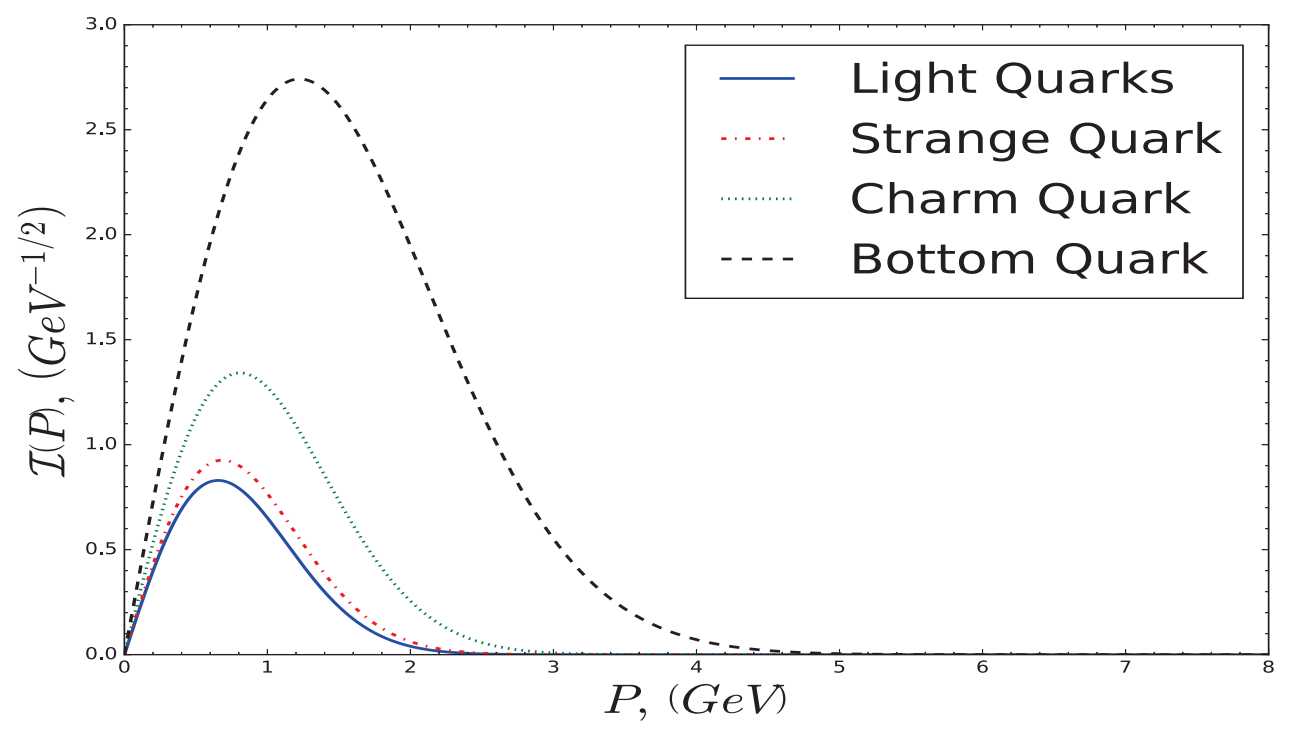

Figure 3.5: Overlap integral of creating a $q \bar{q}$ pair for various choices of $q$. Values for quark masses are taken from the fourth column of table 3.1. The larger amplitude and thus preference of the matrix element for heavier quarks is clear. The quark radius $\left(r_{q}\right)$ is set to zero and $\beta_{\text {eff }}=0.5 \mathrm{GeV}$.

the previous section. Constituent masses in Eq. 2.23 show up in the form of ratios $r \equiv \frac{m_{i}}{m_{i}+m_{j}}$. Taking equal masses turns this ratio into $\frac{1}{2}$ which can be considered an upper bound. We also plot the $1 S \rightarrow 1 S+1 S$ momentum overlap integral for all possible quarks in the vertex in Fig. 3.5. The severe decrease in the magnitude of $\mathcal{I}(P)$ both in Fig. 3.4 and in Fig. 3.5 when we use unequal masses for the constituent quarks as opposed to assuming perfect symmetry between all 5 quarks and taking their mass to be the same, does not necessarily imply that heavier mesons (for example, bottomonium mesons in the loop $b \bar{b} \rightarrow b \bar{b}+\bar{b} b$ ) will contribute more to mass corrections. The momentum overlap integral is still only in the numerator of the integrand of Eq. 2.11 and mesons with two heavy quarks are generally quite heavy themselves. The effect of the mass of the mesons in the loop shows up in the denominator and does ensure that heavier mesons will have their contributions suppressed. The change of 


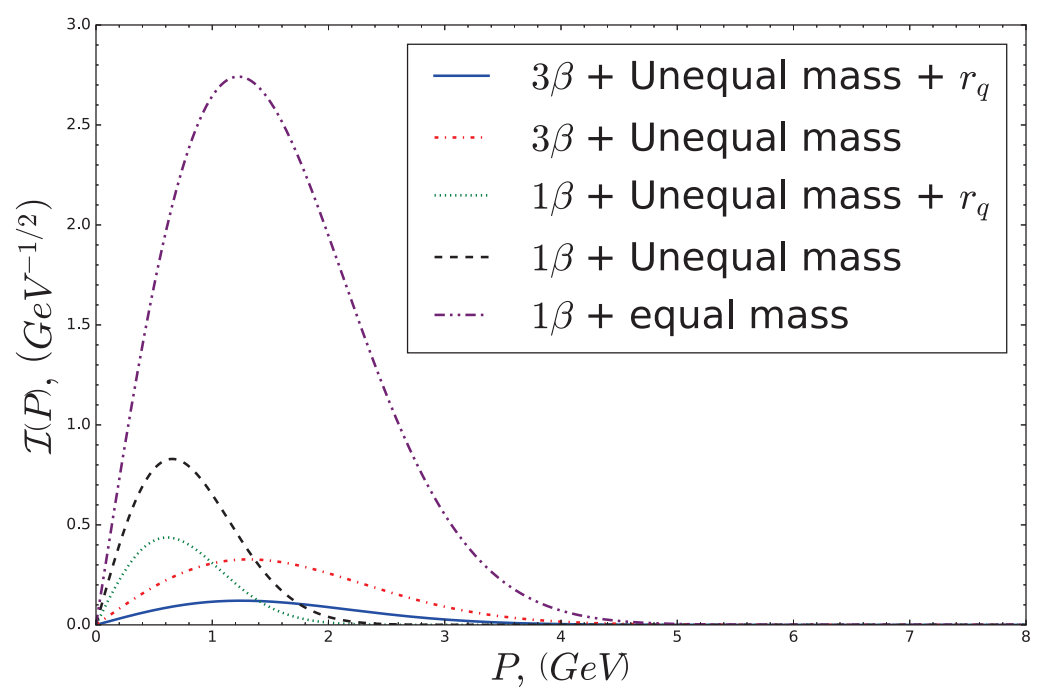

(a) $1 S \rightarrow 1 S+1 S$

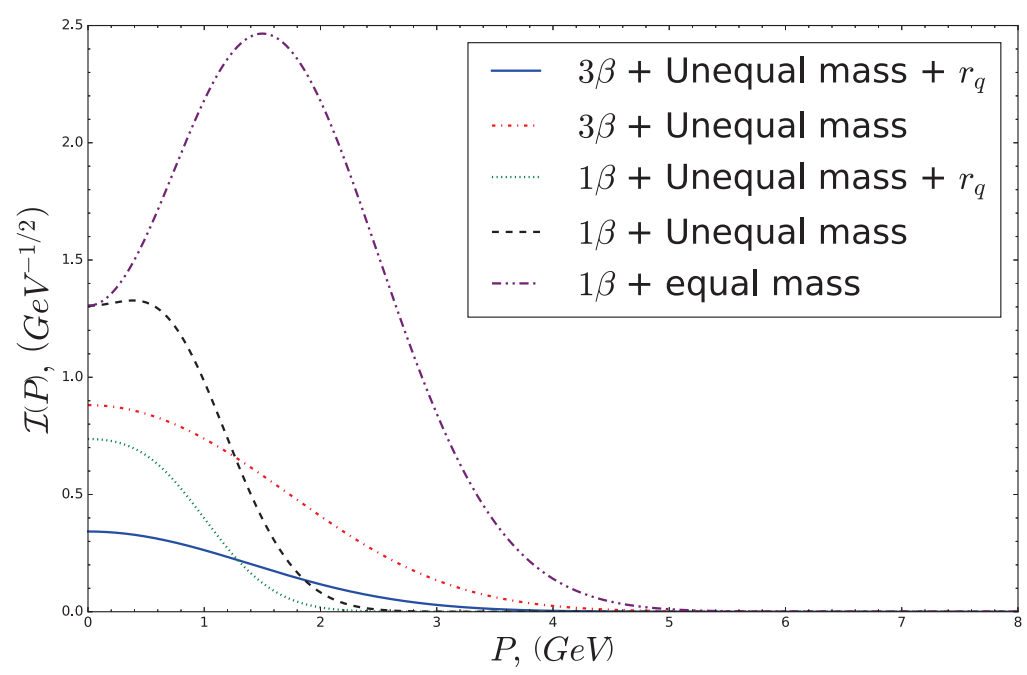

(b) $1 S \rightarrow 1 S+1 P$

Figure 3.6: Figure summarizing the effect of various parameters on the shape of two hypothetical momentum overlap integrals (or matrix element up to a multiplicative constant). The parameter values are from the fourth column of table 3.1 for $\beta_{\text {eff }}$ and $r_{q}$, and table 4.1 for the final state $\beta$ values (using $\beta$ for the $B^{+}$ meson) in the unequal $\beta$ case and 4.2 (using $\beta$ for the $\Upsilon(1 S)$ ). We are assuming light-quark pair creation (up or down quarks). 
$\gamma$ to $\gamma_{0} \frac{m_{n}}{m_{q}}$ which we discussed in chapter 2 also helps in this suppression.

Finally we can consider the effect of unequal wave function parameters. As explained before, especially in heavy quark spectroscopy, the parent state occupies a smaller volume than the final states. As such, one would expect the details of the wave function to become quite important particularly for states close to a decay threshold. This is due to the fact that using fitted $\beta$ values for different states would better capture the details of the wave function and its size than a simple, universal effective parameter being used for objects that belong to different spectra and have different sizes. Fig. 3.6 illustrates this point. The effect of including more realistic wave functions is significant in both plots and contributions of both low and high momenta are significantly suppressed. This is rather good news as it implies that it is likely that in summing over various contributions to mass correction for a given state, each correction will be small and higher angular and radial excitations will contribute less and less.

\subsection{Summary of Parameter Dependence}

We began this chapter aiming to study the numerator of $\Omega(E)$ and its dependence on the parameters of the coupled channel model. The various approximations we considered included the effect of the constituent quark masses, the harmonic oscillator parameter and the quark form factor.

In general we observe that the more details that are taken into account in calculations of momentum overlap integrals, the smaller their values become. We do not expect this to be true for all states, certainly not the states that are very close to a threshold or have many different thresholds around them. However, it is a fair starting point to have in mind when we study the mass corrections to the bottomonium 
and charm-strange spectra.

Specifically, we find that the inclusion of the quark form factor in the operator regulates the higher momentum contributions to the mass correction, once the momentum overlap integral and the re-coupling coefficients are taken into account. We will study this effect in the context of total mass corrections from $1 S+1 S$ mesons to bottomonium and the charm-strange spectrum. We will also expand that study for a low lying bottomonium state, the $\Upsilon(1 S)$ by considering the addition of excited bottom and bottom strange mesons (higher $\mathrm{n}$ and $\mathrm{L}$ ) in the loop. This will help us determine the usefulness or the necessity of such a form factor in calculations of mass corrections.

The wave function $\beta$ parameter studies show the sensitivity of the momentum overlap integral to the details of the wave function. This is important since the momentum integrals themselves become integrands in order to calculate mass corrections and as such, one would expect the details of the wave function to play a significant role particularly for states that are close in mass to thershold. In order to say more about this topic, we need to study the mass corrections which is the subject of the next two chapters.

Finally, the effect of the constituent mass was mostly to illustrate the necessity for the modification of $\gamma_{0}$ to suppress heavy quark production. The equal mass approximation is not usually made in the literature for mass correction or width calculations except for the light meson sector. However, given that mass ratios appear in the momentum overlap integral expression it is important to consider the dependence of the vertex on the mass of the created quark. Our study indicates that the modification, $\gamma \rightarrow \gamma_{0} \frac{m_{q}}{m_{n}}$, is essential to correct for the undue preference that the original QPC model formulation has for heavy quark pair creation. 


\section{Chapter 4}

\section{Bottomonium Spectrum}

Up to this point we discussed various parts of the mass correction function, $\Omega(E)$. It is now time to apply the model to several states in the bottomonium system, turn the handle and see what the model gives. Our goal is not to make physical calculations of masses or mass corrections but rather to understand the behaviour of the model. That means whether the sums that are taken to be convergent do converge or whether certain approximations made by various authors and calculations are sensible. It should be noted that in our studies we always use experimental masses for all intermediate mesons if available and if not will use the predicted masses of the Godfrey-Isgur (GI) relativized quark model [45]. For the bottomonium levels presented here, all "bare" masses are the predicted masses from the GI model. The bare masses are also where we evaluate the mass correction function.

Bottomonium is chosen to be the first system we consider since given the high mass of the bottom quark, a non-relativistic calculation is a better approximation for its bound states than for other mesonic spectra. Furthermore, as the open flavour threshold in bottomonium is fairly high there are many sub-threshold, stable states for which we would expect the meson-meson corrections and wave function components to be small. That is to say, we expect the quark anti-quark picture of mesons to be 
Table 4.1: $1 S$ B-mesons used in the calculations of this section. Masses are from Ref. [12] and $\beta$ values are acquired using the GI model for Ref. [45]. The antiparticles are omitted as their relevant properties are identical. For example, the properties of $B^{-}$are identical to that of $B^{+}$except for the quark content which is $\bar{u} b$ and the electric charge.

\begin{tabular}{ccccc}
\hline Meson & Quark Content & Spectroscopic Notation & Mass $(\mathrm{GeV})$ & $\beta(\mathrm{GeV})$ \\
\hline$B^{+}$ & $u \bar{b}$ & $1^{1} S_{0}$ & 5.27926 & 0.579659 \\
$B^{0}$ & $d \bar{b}$ & $1^{1} S_{0}$ & 5.27958 & 0.579659 \\
$B^{+*}$ & $u \bar{b}$ & $1^{3} S_{1}$ & 5.3252 & 0.5419 \\
$B^{0 *}$ & $d \bar{b}$ & $1^{3} S_{1}$ & 5.3252 & 0.5419 \\
$B_{s}^{0}$ & $s \bar{b}$ & $1^{1} S_{0}$ & 5.36677 & 0.6362 \\
$B_{s}^{0 *}$ & $s \bar{b}$ & $1^{3} S_{1}$ & 5.4154 & 0.5946 \\
\hline
\end{tabular}

best realized in the bottomonium system with limited complexity introduced by the coupled channel effects.

\subsection{Coupling to intermediate low lying S-waves}

Most studies of coupled channel loop corrections to various mesonic sectors perform their calculations with the assumption that the most significant contributions to the total mass correction comes from $1 S+1 S$ loops and that excited meson loops can be ignored. Ref. [67] is an exception to this statement. While we will study this specific assumption later, we will begin our study of loop corrections with only ground state S-waves in the loop.

It should also be noted that we will only present results for a representative sample of bottomonium states and provide the full table of results in Appendix $\mathrm{C}$ for completeness and future replication of our work. The bottom and bottom strange 
Table 4.2: Properties of the bottomonium states used in this chapter. Theoretical masses are obtained from the GI model, given in Ref. [45] while the experimental masses are from the Particle Data group in Ref. [12].

\begin{tabular}{ccccc}
\hline Meson & $\begin{array}{c}\text { Spectroscopic } \\
\text { Notation }\end{array}$ & $\begin{array}{c}\text { Mass }(\mathrm{GeV}) \\
\text { Theoretical }\end{array}$ & $\begin{array}{c}\text { Mass }(\mathrm{GeV}) \\
\text { Experimental }\end{array}$ & $\beta(\mathrm{GeV})$ \\
\hline$\Upsilon(1 S)$ & $1^{3} S_{1}$ & 9.4649 & 9.46030 & 1.1568 \\
$\eta_{b}(1 S)$ & $1^{1} S_{0}$ & 9.4017 & 9.3980 & 1.2690 \\
$\chi_{b 2}(1 P)$ & $1^{3} P_{2}$ & 9.8971 & 9.91221 & 0.8580 \\
$\chi_{b 1}(1 P)$ & $1^{3} P_{1}$ & 9.8764 & 9.89278 & 0.8891 \\
$\chi_{b 0}(1 P)$ & $1^{3} P_{0}$ & 9.8469 & 9.85944 & 0.9320 \\
$h_{b 1}(1 P)$ & $1^{1} P_{1}$ & 9.8821 & 9.8993 & 0.8798 \\
\hline
\end{tabular}

mesons used in the loop as well as their properties are given in table 4.1 and the bottomonium states that we study here are given in table 4.2.

As we saw in chapters 2 and 3, there are many different approximations that can be made in this model. Here we study four variations:

1. Equal $\beta$ without a quark form factor denoted as $1 \beta$

2. Equal $\beta$ with a quark form factor denoted as $1 \beta+r_{q}$

3. Un-equal $\beta$ without a quark form factor denoted as $3 \beta$

4. Un-equal $\beta$ with a quark form factor denoted as $3 \beta+r_{q}$.

From our discussion in chapter 3 we already expect the scenarios with a quark form factor to have reduced contributions relative to their equivalent without a form factor. Also the unequal $\beta$ case is expected to give a smaller contribution, in general, relative to the equal $\beta$ case for mesons with heavy quarks because it is a better approximation of the wave functions involved and better captures the details of the overlap integrals. 


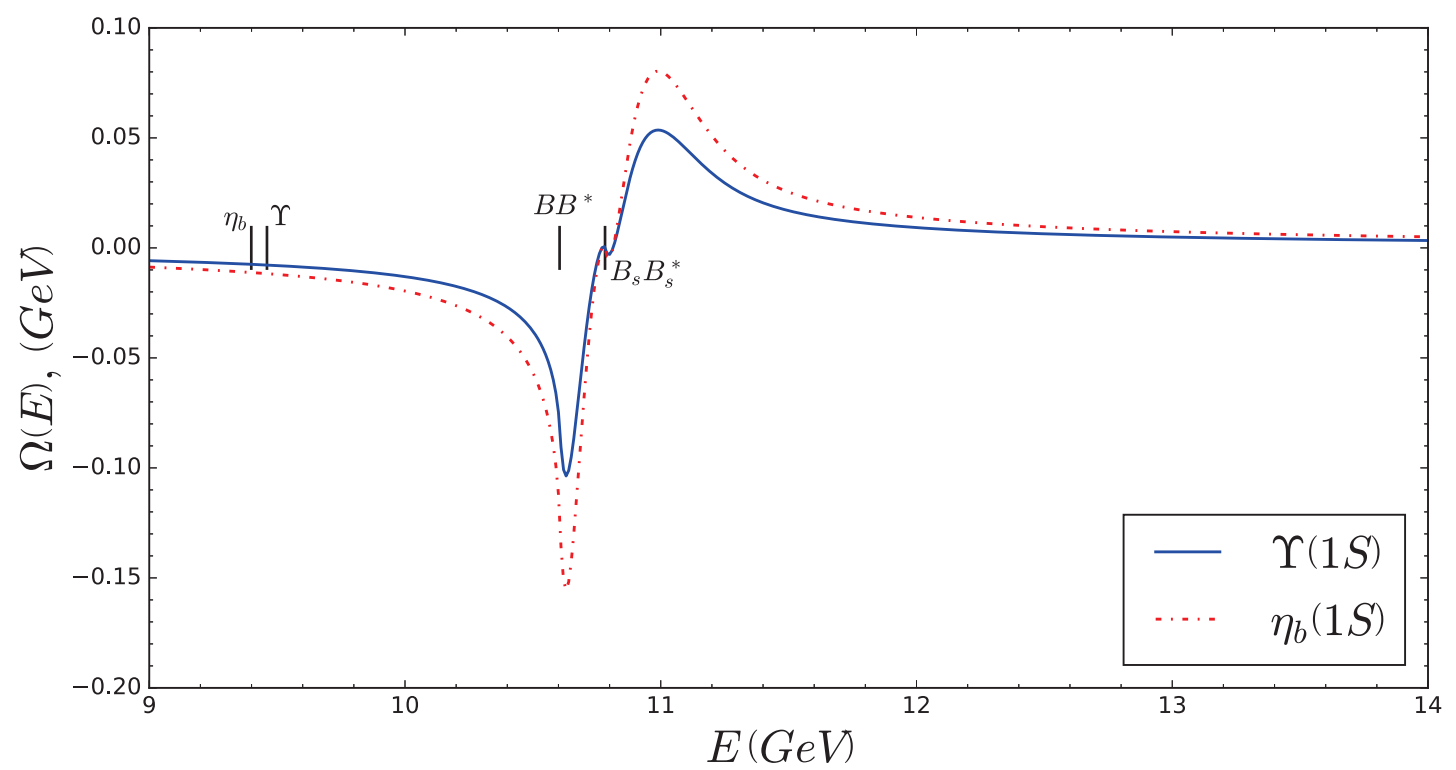

(a) Equal $\beta$ with form factor

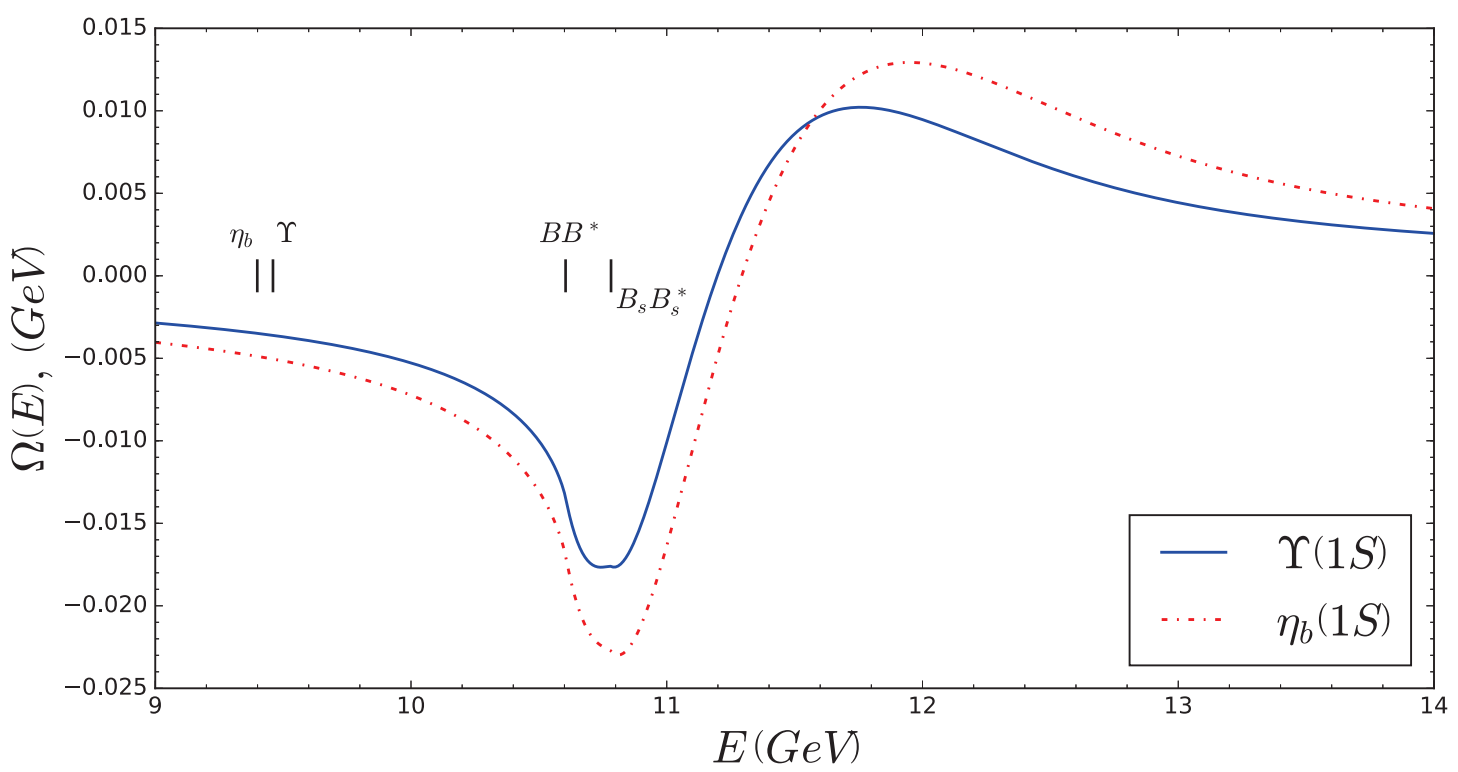

(b) Unequal $\beta$ with form factor

Figure 4.1: Mass correction for $\Upsilon(1 S)$ and $\eta_{b}(1 S)$ via coupling to $B^{+}+B^{-*}\left(B B^{*}\right)$ and $B_{s}^{0}+\bar{B}_{s}^{0 *}\left(B_{s} B_{s}^{*}\right)$ as a function of the mass of the two bottomonium states. The vertical lines denote the physical masses of $\eta_{b}(1 S)$ and $\Upsilon(1 S)$ along with the thresholds of $B B^{*}$ and $B_{s} B_{s}^{*}$, respectively. 
Table 4.3: Comparison of mass corrections from individual bottom and bottomstrange channels to the $1 S$ and $1 P$ bottomonium states. The first number in each column is the $1 \beta+r_{q}$ contribution while the second column is the $3 \beta+r_{q}$ result. For clarity, we denote various meson-meson channel contributions with shorthand form i.e. $B B \equiv B^{+}+B^{-}$and $B^{0}+\bar{B}^{0}$ and so on. A negative sign is factored out from all the numbers in this table.

\begin{tabular}{lllllllllllllll}
\hline \multirow{2}{*}{ State } & \multicolumn{10}{c}{ Channel Mass Correction $(\mathrm{MeV})$} \\
\cline { 2 - 6 } & \multicolumn{1}{c}{$B B$} & \multicolumn{1}{c}{$B B^{*}$} & \multicolumn{1}{c}{$B^{*} B^{*}$} & \multicolumn{1}{c}{$B_{s} B_{s}$} & $B_{s} B_{s}^{*}$ & $B_{s}^{*} B_{s}^{*}$ \\
\hline$\Upsilon\left(1^{3} S_{1}\right)$ & 7.62 & 2.50 & 29.63 & 10.22 & 50.10 & 18.43 & 1.56 & 0.51 & 6.04 & 2.13 & 10.24 & 3.94 \\
$\eta_{b}\left(1^{1} S_{0}\right)$ & 0.00 & 0.00 & 42.38 & 13.69 & 41.01 & 14.20 & 0.00 & 0.00 & 8.69 & 2.93 & 8.43 & 3.12 \\
$\chi_{b 2}\left(1^{3} P_{2}\right)$ & 16.24 & 7.24 & 46.32 & 21.83 & 78.90 & 36.37 & 3.11 & 1.27 & 8.92 & 3.96 & 13.84 & 6.25 \\
$\chi_{b 1}\left(1^{3} P_{1}\right)$ & 0.00 & 0.00 & 66.05 & 27.50 & 72.05 & 35.06 & 0.00 & 0.00 & 11.27 & 4.54 & 13.99 & 6.62 \\
$\chi_{b 0}\left(1^{3} P_{0}\right)$ & 31.29 & 11.24 & 0.00 & 0.00 & 102.62 & 47.18 & 4.90 & 1.68 & 0.00 & 0.00 & 19.69 & 8.96 \\
$h_{b 1}\left(1^{1} P_{1}\right)$ & 0.00 & 0.00 & 71.23 & 31.55 & 67.78 & 31.62 & 0.00 & 0.00 & 13.00 & 5.52 & 12.45 & 5.70 \\
\hline
\end{tabular}

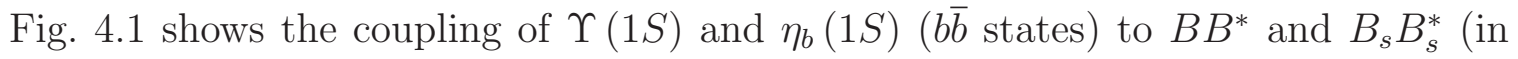
short hand form, see the caption of Fig. 4.1 for explanation). In the first channel we create a light quark pair and a strange pair in the second one. These two channels are chosen as they are the first two-meson channels which couple to both $1 S$ states. Another reason to choose these states was that their thresholds are relatively apart from each other. This gives us an opportunity to also study the effect of a channel opening. The x-axis of the plot is the naive "mass" of the bottomonium states (either $\Upsilon$ or $\eta_{b}$ ) which we let vary. The physical masses and the thresholds are denoted by four vertical lines in the plot. The fact that the two states receive similar corrections is expected. This was proven by Swanson and Barnes in Ref. [24] as a loop theorem in the limiting case where the states in the multiplet are degenerate and coupling to a complete set of intermediate states (mesons within the loop). In that limit, Swanson et al. [24] found that the individual members of the parent multiplet will receive identical mass corrections. 
Since in our work here we are not operating in the degenerate mass case, there will be some difference in the size of the correction between the members of the multiplet. Furthermore, in our plots we are including only two meson loops and the theorem applies to the "total" contribution since not all members of the "parent" multiplet will couple to all members of the intermediate states. As such, some channels will contribute more to one member while other channels may not couple to it at all. This can be clearly seen in table 4.3 where we give the detailed breakdown of the coupling of the complete set of ground state $1 S$ bottom and bottom strange mesons to the $1 S$ and $1 P$ bottomonium multiplets.

Continuing with the general shape of Fig. 4.1 we also see that as we approach the first threshold, $B B^{*}$, the rate of mass correction from that channel increases dramatically. The opening of a new channel induces a kink in the mass correction function with the effect being more pronounced in the $1 \beta+r_{q}$. Denote the loop correction function due to the first threshold $\left(B B^{*}\right)$ as $\Omega_{1}(E)$ and the equivalent for $B_{s} B_{s}^{*}$ to be $\Omega_{2}(E)$. In the equal $\beta$ approximation, the matrix element is simplified to the point that the numerators of $\Omega_{1}(E)$ and $\Omega_{2}(E)$ are quite similar with the difference between the two coming from their quark masses as the quantum numbers are the same. So the denominators of $\Omega_{1}(E)$ and $\Omega_{2}(E)$ start playing an important role in setting the relative strength. Since close to the $B_{s} B_{s}^{*}$ channel we are further away from the $B B^{*}$ threshold, the denominator of $\Omega_{1}$ is no longer approaching zero and has only a singular point while the exact opposite is happening in the denominator of $\Omega_{2}$, leading to increased contributions from that channel. We also see that as the naive state moves beyond the second threshold, contributions from both channels become smaller, turning positive and if the state moves too far away from the two continuum channels it will eventually decouple from them. 
Table 4.4: Comparison of total mass correction from the coupling of low lying bottomonium states $(1 S$ and $1 P)$ to $1 S+1 S$ intermediate bottom mesons including the $u, d$ and the $s$ quarks. All values are in $\mathrm{MeV}$. We used the parameters of tables 3.1 (fourth column), 4.1 and 4.2 .

\begin{tabular}{ccccc}
\hline State & $\Delta M(1 \beta)$ & $\Delta M\left(1 \beta+r_{q}\right)$ & $\Delta M(3 \beta)$ & $\Delta M\left(3 \beta+r_{q}\right)$ \\
\hline$\Upsilon(1 S)$ & -474.36 & -105.26 & -432.59 & -37.73 \\
$\eta_{b}(1 S)$ & -453.40 & -100.51 & -399.13 & -33.93 \\
$\chi_{b 2}(1 P)$ & -655.85 & -167.32 & -733.40 & -76.91 \\
$\chi_{b 1}(1 P)$ & -640.64 & -163.37 & -704.55 & -73.73 \\
$\chi_{b 0}(1 P)$ & -621.99 & -158.49 & -673.50 & -69.05 \\
$h_{b 1}(1 P)$ & -644.90 & -164.46 & -712.25 & -74.39 \\
\hline
\end{tabular}

Table 4.4 compares the total mass correction arising from coupling to ground state bottom and bottom strange mesons. From all four columns we can see that the overall mass contributions to members of the same multiplet are similar which is a verification of the loop theorem of Swanson, as mentioned before. The overall reduction in the total mass correction due to the inclusion of the quark form factor is also very clear. There is an issue with the results as the predicted naive masses of the $1 P$ multiplet are lower than the experimental masses and the loop corrections push the predicted mass even lower. This highlights that this mass correction calculation is only the first step of what should be a fit, assuming that more excited mesons in the loop have vanishing contributions.

We can now compare our results for these states with some published values in the literature. One natural comparison is with the work of Ferretti et al. [49] (second column of table 4.5) as we are using their parameters (with some modification). Table 4.5 compares our results for unequal wave function parameters including a quark form factor with those of other published works. While the actual value of the 
Table 4.5: Comparison between previously published results for loop mass correction calculations in the literature and the work of this thesis. We compared our $3 \beta+r_{q}$ using parameters of table 3.1 to that of previously published works by various authors. In the third column, the $\Upsilon$ and $\eta_{b}$ results are taken from [26] while the $1 P$ results are from [27]. It should be noted that in [26] the two states in the $1 S$ multiplet are taken to be degenerate in mass, hence the equal induced mass shift. RY column is the calculation of this work. All values are in $\mathrm{MeV}$.

\begin{tabular}{cccccc}
\hline State & $\Delta M([49])$ & $\Delta M([47])$ & $\Delta M([27],[26])$ & $\Delta M([62])$ & $\Delta M(\mathrm{RY})$ \\
\hline$\Upsilon(1 S)$ & -68 & -22.8 & -30 & -58.25 & -37.73 \\
$\eta_{b}(1 S)$ & -63 & -22 & -30 & -55.49 & -33.93 \\
$\chi_{b 2}\left(1^{3} P_{2}\right)$ & -115 & -36.4 & -45 & -87.33 & -76.91 \\
$\chi_{b 1}\left(1^{3} P_{1}\right)$ & -112 & -35.5 & -44 & -84.78 & -73.73 \\
$\chi_{b 0}\left(1^{3} P_{0}\right)$ & -107 & -34.6 & -43 & -81.83 & -69.05 \\
$h_{b 1}\left(1^{1} P_{1}\right)$ & -113 & -35.8 & -44 & -85.74 & -74.39 \\
\hline
\end{tabular}

mass corrections seems to span a wide range of numbers, it is important to remember that these corrections are sensitive to the details of the model as well as the masses used for the intermediate states. All the columns in this table still conform to the loop theorem of Ref. [24]. Furthermore within the $1 P$ multiplet all calculations give a smaller correction to the $1^{3} P_{0}$ state while the $1^{3} P_{2}$ state gets the largest correction. $1^{3} P_{1}$ and $1^{1} P_{1}$ states have similar corrections. Similar patterns repeat themselves for higher radial and orbital excitations. We also see that compared to the $1 S$ states, the $1 P$ waves get larger mass corrections. This is again to be expected since in Fig. 4.1 we saw that the closer the state is to the threshold the larger the correction will be and $1 P$ states are around $400-500 \mathrm{MeV}$ closer to the threshold than the $1 S$ waves. This is related to the behaviour of the denominator of Eq. 2.11. We should also mention that our findings here generalize to tables C.3 and C.4.

It is perhaps also helpful to briefly go over the methods used by the sources cited in table 4.5. All of them use the master equation Eq. 2.10 with Eq.2.11. The QPC 
model is also used in all of them to model the strong pair creation vertex and the points of departure between what they did versus what we do are as follows:

- [49] uses a $1 \beta+r_{q}$ scheme, an $S U$ (5) flavour wave function for the produced $q \bar{q}$ pair which means they also include $1 S+1 S$ loops from $B_{c}$ and $b \bar{b}$ states. The contributions of these states is negligible in their calculations. The normalization factor used in their work seems to be different from what we have in Eq. 2.23. In our effort to reproduce their work, we found that $\sqrt{128 \pi}$ seems to better match their results. They also use a modified version of the GI model and take the masses of bottom mesons of different isospins to be equal.

- [47] uses a non-relativistic naive potential model and present their results in two ways: one using $\mathrm{SHO}$ wave functions and another using the Gaussian Expansion Method [68] to faithfully fit the numerical wavefunction and get analytical expression for use in matrix element and mass correction calculations. Their aim is to check for the effect of using realistic wavefunctions. They make no indication whether the B-meson masses used in their calculations are set to be equal for different isospins or not (we infer that that is what they did).

- $[26,27]$ also use a non-relativistic naive potential and no isospin effects in the masses of the B-mesons. Furthermore as mentioned in table 4.5 they take the masses of $\Upsilon(1 S)$ and $\eta_{b}(1 S)$ to be degenerate.

- [62] uses a $1 \beta$ scheme with no quark form factor and $\beta_{\text {eff }}=0.4 \mathrm{GeV}$. The naive potential used is a non-relativistic one. B-meson masses do not include isospin effects.

Ultimately all the authors of $[26,27,47,49,62]$ aim to fit the bottomonium spectrum using coupled-channel effects. We mentioned one way that the fit can be performed 
in chapter 2 which is to take the bare mass to be a first estimate of physical mass. Then Eq. 2.10 is called with $M=m_{0}+\Omega\left(m_{0}\right)$ and this process is repeated for $M^{\prime}=$ $m_{0}+\Omega(M)$ until convergence is achieved. Alternatively, one can use the physical masses of a few low lying states and fit them for the naive potential parameters. Then using these parameters and calculating the mass correction at the physical mass, the required bare masses, $m_{0}$, can be calculated. The potential is then fitted with these bare masses for a rescaling of the naive potential model parameters and separating the coupled channel effects from the traditional $q \bar{q}$ interactions. Our results are the first step of the former method.

\section{$4.2 \Upsilon(1 S)$ as Test of Convergence}

In this section we take one member of the $1 S$ multiplet of bottomonium and add excited bottom and bottom strange mesons in the loop. An important assumption made in coupled channel calculations is the idea that excited states in the loop will have negligible contributions and as such taking the $1 S+1 S$ states is enough to capture the coupled channel effect. This issue had received some attention previously [52] for a different study and it was determined that for the series to converge, it is necessary to include a quark form factor. More recently authors of Ref. [67] looked into the addition of excited mesons in the loop and determined that even with a quark form factor, the series does not converge fast enough and that the $1 P+1 P$ contributions are in fact larger than the $1 S+1 S$ corrections. In their calculations, they take the masses of B-mesons belonging to the same multiplet to be equal and only go so far as the $B(2 S)$ contributions, performing the calculations for $\Upsilon(n S)$ for $n=(1,2,3,4,5,6)$ using $r_{q}$ and the Gaussian Expansion Method to fit their wavefunctions, which is roughly similar to our $3 \beta+r_{q}$ case. We performed our own 
Table 4.6: Contributions of the radially excited S-waves to the loop mass correction of $\Upsilon(1 S)$ for a $1 \beta$ scenario. There is no quark form factor included. The table should be read as row + column for the states within the loop. For example, the first entry is the contribution from $1 S+1 S$. All values are in $\mathrm{MeV}$.

\begin{tabular}{cccccc}
\hline & $1 \mathrm{~S}$ & $2 \mathrm{~S}$ & $3 \mathrm{~S}$ & $4 \mathrm{~S}$ & $5 \mathrm{~S}$ \\
\hline $1 \mathrm{~S}$ & -474.36 & -111.92 & -19.94 & -2.72 & -0.26 \\
$2 \mathrm{~S}$ & & -458.59 & -108.38 & -30.68 & -6.40 \\
$3 \mathrm{~S}$ & & & -397.44 & -109.10 & -36.36 \\
$4 \mathrm{~S}$ & & & & -342.28 & -109.94 \\
$5 \mathrm{~S}$ & & & & & -298.13 \\
\hline
\end{tabular}

Table 4.7: Contributions of the radially excited P-waves to the loop mass correction of $\Upsilon(1 S)$ for a $1 \beta$ scenario. There is no quark form factor included. The table should be read as row + column for the states within the loop. For example, the first entry is the contribution from $1 P+1 P$. All values are in $\mathrm{MeV}$.

\begin{tabular}{cccc}
\hline & $1 \mathrm{P}$ & $2 \mathrm{P}$ & $3 \mathrm{P}$ \\
\hline $1 \mathrm{P}$ & -2271.88 & -553.88 & -94.52 \\
$2 \mathrm{P}$ & & -2276.02 & -682.32 \\
$3 \mathrm{P}$ & & & -2153.78 \\
\hline
\end{tabular}

calculations, in a $1 \beta+r_{q}$ scenario for corrections to $\Upsilon(1 S)$ with loops including $1-5 S$, $1-4 P, 1-2 D$ and $1 F$ bottom and bottom-strange mesons. We also performed the calculation for a smaller subset $(1-2 S$ and $1 P$ mesons in the loop) for a $3 \beta$ with and without $r_{q}$ which we present here. For the $1 \beta$ case we only present the S-wave and $\mathrm{P}$-wave results here and include the rest in Appendix C.

We begin with an equal $\beta$ approximation. From tables 4.6 and 4.7 we see that going across a row (i.e. $1 S+n S$ ) the contributions fall while they rise moving down a column. This is problematic as clearly the approximation of $1 S+1 S$ states in 
Table 4.8: Contributions of the radially excited S-waves to the mass correction of $\Upsilon(1 S)$ in $1 \beta$ scheme with quark form factor included. The table should be read as row + column for the states within the loop. For example the first entry is the contribution from $1 S+1 S$. All values are in $\mathrm{MeV}$.

\begin{tabular}{cccccc}
\hline & $1 \mathrm{~S}$ & $2 \mathrm{~S}$ & $3 \mathrm{~S}$ & $4 \mathrm{~S}$ & $5 \mathrm{~S}$ \\
\hline $1 \mathrm{~S}$ & -105.26 & -2.68 & -1.07 & -1.26 & -0.60 \\
$2 \mathrm{~S}$ & & -10.94 & -4.44 & -0.20 & -0.32 \\
$3 \mathrm{~S}$ & & & -5.17 & -4.15 & -0.56 \\
$4 \mathrm{~S}$ & & & & -3.13 & -3.18 \\
$5 \mathrm{~S}$ & & & & & -2.06 \\
\hline
\end{tabular}

the loop is not enough. The issue is not helped by the significant contributions that the $n P+n^{\prime} P$ states make within the loop. These result clearly demonstrate an issue we discussed before, that the higher momentum contributions in the loop need to be suppressed much more than the traditional QPC model does on its own. Furthermore, the fact that radially excited bottom mesons have contributions that are comparable to the $1 S+1 S$, even when we go as high $n=5$ shows the need for a better approximation and modeling of the wave functions. This is compounded by what is observed in table C.1 where we can see that the corrections of loops from $D$ and $F$ waves are also extremely large and in fact overwhelm the contributions from $1 S+1 S$ loops. This issue arises from the fact that the $\beta$ values for loop mesons, regardless of their radial or angular quantum numbers, are around $0.45 \mathrm{GeV}$ and change very slowly as we go to more excited states. As such the contributions decrease slowly as we increase $n$ in $n S+n S$ and actually get larger as we move down the columns of tables 4.6 and 4.7 since the wave function parameters of the two mesons in the loop approach each other, increasing their overlap.

The addition of the quark form factor in tables 4.8 and 4.9 while reducing the 
Table 4.9: Contributions of the radially excited P-waves to the mass correction of $\Upsilon(1 S)$ including a quark form factor. The table should be read as row + column for the states within the loop. So for example the first entry is the contribution from $1 P+1 P$. All values are in $\mathrm{MeV}$.

\begin{tabular}{cccc}
\hline & $1 \mathrm{P}$ & $2 \mathrm{P}$ & $3 \mathrm{P}$ \\
\hline $1 \mathrm{P}$ & -152.17 & -30.28 & -1.60 \\
$2 \mathrm{P}$ & & -36.21 & -22.14 \\
$3 \mathrm{P}$ & & & -17.21 \\
\hline
\end{tabular}

Table 4.10: Contributions of the radially excited S-waves to the mass correction of $\Upsilon(1 S)$ with unequal $\beta$ and the quark form factor included. The table should be read as row + column for the states within the loop. So for example the first entry is the contribution from $1 S+1 S$. All values are in $\mathrm{MeV}$.

\begin{tabular}{cccc}
\hline & $1 \mathrm{~S}$ & $2 \mathrm{~S}$ & $3 \mathrm{~S}$ \\
\hline $1 \mathrm{~S}$ & -37.75 & -1.34 & -0.04 \\
$2 \mathrm{~S}$ & & -12.41 & -2.66 \\
$3 \mathrm{~S}$ & & & -6.59 \\
\hline
\end{tabular}

overall magnitude of contributions does not seem to improve the convergence issue as clearly demonstrated by table C.2. Loop corrections due to higher S-waves do fall off faster but they are still fairly large up to $5 S+5 S$ loops and $D+F$ and $D+D$ contributions are still very significant. The same can be said for the P-wave contributions.

We also tested this calculation using unequal $\beta$ and a quark form factor in tables 4.10 and 4.11. Again we see an overall reduction in the magnitude of the contributions but the convergence is still slow and the $1 P+1 P$ contributions are twice as large as the $1 S+1 S$.

The pattern observed in the calculation implies that the quark form factor and 
Table 4.11: Contributions of the radially excited P-waves to the mass correction of $\Upsilon(1 S)$ with unequal $\beta$ and including a quark form factor. So for example the first entry is the contribution from $1 P+1 P$. All values are in $\mathrm{MeV}$.

\begin{tabular}{ccc}
\hline & $1 \mathrm{P}$ & $2 \mathrm{P}$ \\
\hline $1 \mathrm{P}$ & -60.07 & -6.30 \\
$2 \mathrm{P}$ & & -26.47 \\
\hline
\end{tabular}

a more accurate representation of the wave function, at least for the bottomonium system, seem to be quite important and necessary for a converging sum. Given the large effect of the addition of a form factor alone, we can reasonably conclude that the QPC does in fact overestimate the contributions of larger momenta. For a width calculation, since the function will be evaluated at a single point, this is not an important issue but for a mass correction computation, specially if one is close to a threshold with a vanishing denominator the inclusion of a quark form factor becomes crucial. The inclusion of unequal $\beta$ 's as our way of accounting for the complexities of the wave functions also did help with reduction of corrections due to excited mesons in the loop. It also points towards the idea that perhaps for loop calculations one needs a more precise representation of the wavefunctions. 


\section{Chapter 5}

\section{Charm-Strange Spectrum}

The charm-strange sector is home to two interesting states: the $D_{s}(2317)[10]$ and the $D_{s}(2460)$ [69]. As was mentioned before in chapters 1 and 2, it was the discovery of these two states (starting with the $D_{s}(2317)$ ) that made mesonic loop corrections a more widely studied topic. In this chapter we will look at the discovered states in the charm strange sector and calculate loop corrections to the constituent quark model predictions of their masses. Our aim is to study the effect of the loop corrections on these predictions and determine whether the model is in principle capable of adjusting theory predictions to account for the out-of-step states in the charm strange spectrum.

\subsection{Naive predictions}

Charm-strange mesons are a light-heavy quark system. While positronium can be thought of as an analogy to bottomonium or charmonium, the hydrogen atom is a good analogy of cs mesons: a heavy particle defining the centre of the coordinate system with a much lighter particle orbiting it. It is because of this mass difference and the fact that the charm quark is quite heavy relative to up, down and strange quarks that a new symmetry can be introduced; the Heavy Quark Symmetry (HQS) 
[70]. All of these, of course, also apply to the bottom quark so we expect roughly the same behaviour in the bottom and bottom strange meson mass spectra. In the HQS framework, the mass of the heavy $m_{Q}$ is taken to be infinite which means that the heavy quark properties decouple from the system. This is evident from the $1 / m_{i}$ dependence in Eq. 1.5 and it implies that the total spin of the meson, given before as

$$
\mathbf{J}=\mathbf{L}+\left(\mathbf{S}_{Q}+\mathbf{S}_{q}\right)_{S}
$$

as a sum of the angular momentum of the system and the vector sum of the two spins, will now be given as [8]

$$
\mathbf{J}=\mathbf{S}_{Q}+\left(\mathbf{L}+\mathbf{S}_{q}\right)_{j}
$$

With this, mesons with the same $(L, j)$ will form doublets with each member of the doublet corresponding to the two spin configurations of the heavy quark. $L$ is the angular momentum between the two quarks and $j$ is the total spin of the light quark.

Predictions for the masses of the $D_{s}(2317)$ and the $D_{s}(2460)$, two members of the P-wave $(\mathbf{L}=1)$ charm-strange mesons, all fall around 2.48 and $2.55 \mathrm{GeV}[7,65,71$, 72] respectively which is about 150 and $100 \mathrm{MeV}$ greater than the observed physical masses. An important expectation fom Heavy Quark Symmetry is the idea that if one plots the spectra of two similar systems (charmonium vs bottomonium or charmstrange vs charmed) the spectra will match fairly well [70]. In case of the $D_{s}(2317)$ and $D_{s}(2460)$, measured masses are comparable to their counterparts in the charmed quark mesons with the $D_{s}(2317)$ being slightly lighter than its cousin in the charmed mesons $[12,72]$. This behaviour of these two states is indicative of something deeper going on than just the naive potential, specially since the other members of the charmstrange family are explained and fitted fairly well by various naive potential models, 


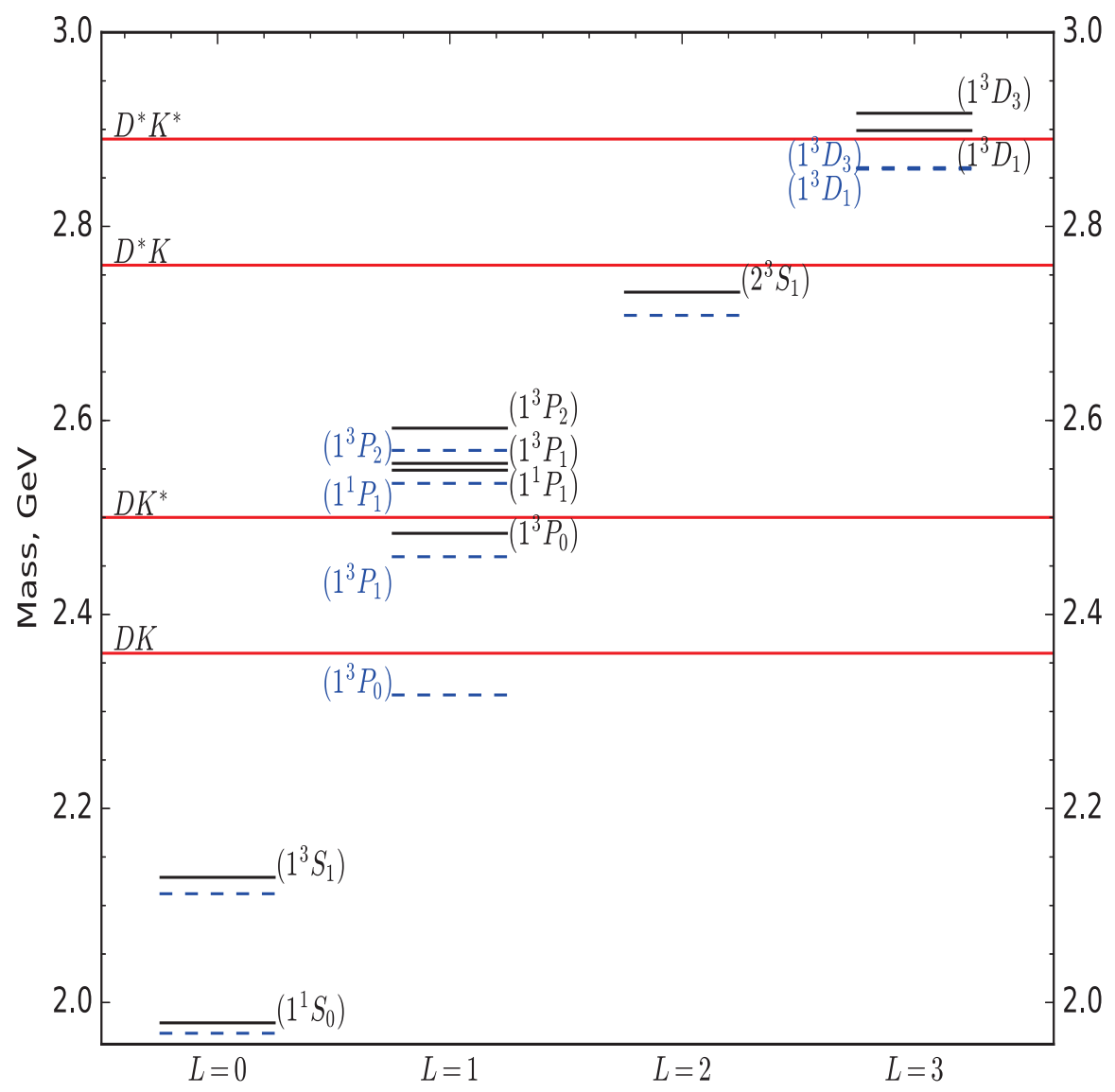

Figure 5.1: Spectrum of the known charm-strange mesons. Blue lines (dashed) are experimental values while black lines (solid) are quark model predictions from Ref. [65]. The experimental labels were not added for the S-waves as they were not necessary. The D-waves become almost degenerate (to within a few $\mathrm{MeV}$ ). The horizontal lines spanning the plot are strong decay thresholds involving $1 S+1 S D$ and $K$ mesons. Thresholds for various isospin combinations $\left(D^{+}+K^{0}\right.$ and $D^{0}+K^{+}$for $D K$ ) are shown as one since their values are also almost equal to within tens of $\mathrm{MeV}$ which would not show on the scale of this plot. The $D K$ threshold is the first open strong decay threshold for a charm-strange meson.

relativized or not. From Fig. 5.1 we can clearly see that while the predictions for the S-wave states are slightly different when compared to experiment, those for the P-wave states clearly do not match the observed spectrum. Naive models predict a P-wave multiplet with masses such that all members can undergo strong decay. This expectation is directly contradicted by the experimental measurements of the masses 
of these states. While the two heavier members of the multiplet $\left(1^{3} P_{2}\right.$ and $\left.1^{1} P_{1}\right)$ are observed with masses lower by $\approx 23$ and $13 \mathrm{MeV}$, respectively, the two lighter states are significantly lower in mass as compared to their predicted levels and as such have their properties altered completely. The $D_{s}(2317)$ which is the $1^{3} P_{0}$ state, goes from a state capable of undergoing strong decay to a state that is completely stable against OZI allowed decays. The typical lifetime of a state that can undergo an OZI-allowed strong decay is $\approx 10^{-25}$ seconds. With the width data of this state and the $D_{s}(2460)$ state from the Particle Data Group ( the full widths of these two states are less than 3.8 and $3.5 \mathrm{MeV}$, respectively) [12] we can estimate the upper bound of the mean life time for both of them. This is done using

$$
\tau=\hbar / \Gamma
$$

where $\Gamma$ is the width, $\tau$ is the mean life time [21] and $\hbar$ is the reduced Planck's constant. The lifetimes come out to $\approx 10^{-22}$ seconds for both states, a three orders of magnitude difference between what was expected and what is observed. It is this unexpected behaviour that prompted theorists to reconsider how quark model calculations are performed.

The same issue but at a smaller scale can be seen in the D-wave case. The two currently discovered states of this multiplet $[73,74]$ come out nearly degenerate to each other with the mass difference currently standing at $\approx 1.5 \mathrm{MeV}$. However they are both predicted to be slightly above $D^{*} K^{*}$ threshold with a mass difference of about $20 \mathrm{MeV}$ between them. In table 5.1 we make explicit the mass differences between theoretical expections and observed values. Normally one does not expect quark model calculations to be accurate to better than $\approx 10-20 \mathrm{MeV}[7]$. As such the $\Delta M$ shown for $1^{3} S_{1}, 1^{1} S_{0}, 2^{3} S_{1}, 1^{3} P_{2}$ and $1^{1} P_{1}$ are not entirely unreasonable. The 
Table 5.1: Comparison of masses and mass differences between theoretical predictions and experimental observations for the known $D_{s}$ states. Theoretical masses are from the GI model [65] and experimental masses are from [12]. The very large discrepancy between the theory and experiment for $1^{3} P_{0}$ and $1^{3} P_{1}$ states is clearly evident.

\begin{tabular}{cccc}
\hline State & Mass Theory $(\mathrm{MeV})$ & Mass Experiment $(\mathrm{MeV})$ & $\Delta M(\mathrm{MeV})$ \\
\hline $1^{3} S_{1}$ & 2129.0 & 2112.1 & 16.9 \\
$1^{1} S_{0}$ & 1979.0 & 1968.3 & 10.7 \\
$1^{3} P_{2}$ & 2592.0 & 2569.1 & 22.9 \\
$1^{3} P_{1}$ & 2555.7 & 2459.5 & 96.2 \\
$1^{3} P_{0}$ & 2483.6 & 2317.7 & 165.9 \\
$1^{1} P_{1}$ & 2548.7 & 2535.1 & 13.6 \\
$1^{3} D_{1}$ & 2898.8 & 2859 & 39.8 \\
$1^{3} D_{3}$ & 2916.6 & 2860.5 & 56.1 \\
$2^{3} S_{1}$ & 2732.2 & 2708.3 & 23.9 \\
\hline
\end{tabular}

two D-waves have slightly larger discrepancies with their predicted masses and the remaining $\mathrm{P}$-waves, $1^{3} P_{0}$ and $1^{3} P_{1}$ are significantly different from what is expected.

This puzzle of anomalously low masses for the two low lying P-waves has led to various suggestions and models trying to account for it. Overall there are three categories of models aiming to explain the nature of these two states

1. Molecular Interpretation

2. Tetraquark Interpretation

3. Canonical cs mesons modified by coupled channel effects.

All three schemes rely on a coupling of the $D_{s}(2317)$ and the $D_{s}(2460)$ to nearby strong decay thresholds. In the first class of models, the $D_{s}(2317)$ and the $D_{s}(2460)$ are taken as bound molecules of the $D K$ and the $D K^{*}$ systems, respectively. In the 
second scheme, the $D_{s}(2317)$ and the $D_{s}(2460)$ are taken as either pure tetraquarks containing $c \bar{s} u \bar{u}$ (or other similar content) or as tetraquark states mixing with the original $1 P$ multiplet members [75]. We do not consider these two classes of models here. For both of these models, there are research articles published both for and against such interpretations with the final conclusions reached being quite model dependent [76]. We will study the third of these models, the canonical meson with distortions due to coupled channel effects. For a comprehensive overview of the molecular and tetraquark calculations we refer the interested reader to Ref. [8] and references therein.

\subsection{Charm Strange Mesons and the Continuum}

Studies of charm strange mesons in a coupled channel setting have been done by various groups ever since the discovery of the state. The first such calculation was done by Van Beveren and Rupp [77] finding that one can get a low mass charmstrange meson once the coupling to the $D K$ continuum channel is included. Later they performed the same computation for the $D_{s}(2460)$ by accounting for the coupling of the naive state to $D K^{*}[78]$. Since then, more studies have been done on the coupledchannel model of the charm-strange spectrum [31, 79-82] and there are many more studies of these states and we refer to Ref. [8] for a more comprehensive list. The consensus seems to be converging on the importance of the coupled channel effect and the strong coupling of the naive state to the OZI allowed thresholds which induce a large mass correction.

Here we will use the QPC model to calculate mass corrections to the bare masses of the GI model for the $1 S, 2 S, 1 P$ and $1 D$ multiplets. In Ref. [31] it was shown that the Cornell potential (with the Coloumb interaction as well as the linear potential) 
Table 5.2: $\beta$ and mass values for the charm strange mesons used in this work. When referring to a given state, we will use its spectroscopic notation to avoid confusion. The quark content of the presented states is $c \bar{s}$. The antiparticles of these mesons will have identical properties save for their quark content and electric charge and are therefore omitted for clarity. Values taken from Ref. $[65]$.

\begin{tabular}{cccc}
\hline State & $\begin{array}{c}\text { Spectroscopic } \\
\text { Notation }\end{array}$ & Mass $(\mathrm{GeV})$ & $\beta(\mathrm{GeV})$ \\
\hline$D_{s}^{+}$ & $1^{1} S_{0}$ & 1.979 & 0.6511 \\
$D_{s}^{+*}$ & $1^{3} S_{1}$ & 2.129 & 0.5619 \\
\hline$D_{s 0}^{+*}(1 P)$ & $1^{3} P_{0}$ & 2.4836 & 0.5415 \\
$D_{s 1}^{+}(1 P)$ & $1^{1} P_{1}$ & 2.5487 & 0.4976 \\
$D_{s 1}^{+*}(1 P)$ & $1^{3} P_{1}$ & 2.5557 & 0.5048 \\
$D_{s 2}^{+*}(1 P)$ & $1^{3} P_{2}$ & 2.5920 & 0.4641 \\
\hline$D_{s}^{+}(2 S)$ & $2^{1} S_{0}$ & 2.6729 & 0.4752 \\
$D_{s}^{+*}(2 S)$ & $2^{3} S_{1}$ & 2.7322 & 0.4575 \\
\hline$D_{s 1}^{+*}(1 D)$ & $1^{3} D_{1}$ & 2.8988 & 0.4689 \\
$D_{s 2}^{+}(1 D)$ & $1^{1} D_{2}$ & 2.9005 & 0.4440 \\
$D_{s 2}^{+*}(1 D)$ & $1^{3} D_{2}$ & 2.9260 & 0.4483 \\
$D_{s 3}^{+*}(1 P)$ & $1^{3} D_{3}$ & 2.9166 & 0.4263 \\
\hline
\end{tabular}

with a sensible choice of parameters can generate the necessary mass shift for bringing the $1^{3} P_{0}$ state's naive mass prediction into agreement with experiment. However, in that study the authors only considered the mass shift induced in a single state, $1^{3} P_{0}$. This is an assumption that we will examine in this work.

As with our study of the bottomonium sector, there are 4 combinations of parameters than can be studied. Given that the mass difference between a charm strange meson and charmed mesons which will be included in the loops is not as pronounced as that between a bottomonium system and bottom mesons, we expect the equal $\beta$ 
both with and without the quark form factor to be a better approximation than it was in chapter 4.

Since in this chapter we also want explicit mass shifts, we will use a rescaling calculation suggested by Pennington and Wilson in Ref. [83]. We know that all of the infinite tower of two meson states contribute to the real part of the loop mass correction function of all bare states. Furthermore many of these two meson channels are too far above our naive states and most likely contribute only a constant to $\operatorname{Re}[\Omega(E)]$ and nothing to $\operatorname{Im}[\Omega(E)]$ and as such we can think of their contributions as an overall rescaling of the bare mass and not necessarily inducing relative mass shifts. Pennington and Wilson suggested that by subtracting from the total loop mass correction calculation, $\Omega(E)$ at a suitable value for $E$, we can approximately correct for such rescaling and arrive at explicit mass shifts. In this scheme one chooses a mass scale or a reference mass, $m_{\text {ref }}$, often the mass of a low lying state or sum of the masses of constituent quarks in the system and subtracts the mass corrections evaluated at that value from the total mass correction to each state.

$$
\Delta M=\Omega\left(m_{0}\right)-\Omega\left(m_{\text {ref }}\right)
$$

This is in effect a recalibration of the constant term in the naive potential of Eq. 1.2 or Eq. 1.6.

With the data in tables 5.2 and 5.3 we can set up our coupled channel calculation, using the second column of the parameter values in table 3.1. We first present the results for comparison of total mass correction in $1 \beta$ vs $3 \beta$ and $1 \beta+r_{q}$ vs $3 \beta+r_{q}$ configurations without the Pennington subtraction scheme of Eq. 5.4, described above. From table 5.4 we see that unlike the bottomonium case (table 4.4) the unequal $\beta$ 
Table 5.3: $\beta$, quark content and mass information for charmed mesons and kaons used in this work. Masses and $\beta$ values are from Ref. [65]. Given how light the Kaons are and how close together the constituent masses of the light quarks and the strange quark is, taking a single $\beta$ value for these states is a reasonable approximation. For clarity, antiparticles are not shown as they will have identical properties.

\begin{tabular}{ccccc}
\hline State & $\begin{array}{c}\text { Spectroscopic } \\
\text { Notation }\end{array}$ & Quark Content & $\operatorname{Mass}(\mathrm{GeV})$ & $\beta(\mathrm{GeV})$ \\
\hline$D^{+}$ & $1^{1} S_{0}$ & $c \bar{d}$ & 1.86961 & 0.6010 \\
$D^{0}$ & $1^{1} S_{0}$ & $c \bar{u}$ & 1.86484 & 0.6010 \\
$D^{+*}$ & $1^{3} S_{1}$ & $c \bar{d}$ & 2.01026 & 0.5162 \\
$D^{0 *}$ & $1^{3} S_{1}$ & $c \bar{u}$ & 2.00696 & 0.5162 \\
\hline$K^{+}$ & $1^{1} S_{0}$ & $u \bar{s}$ & 0.493677 & 0.4 \\
$K^{0}$ & $1^{1} S_{0}$ & $d \bar{s}$ & 0.497614 & 0.4 \\
$K^{+*}$ & $1^{3} S_{1}$ & $u \bar{s}$ & 0.89166 & 0.4 \\
$K^{0 *}$ & $1^{3} S_{1}$ & $d \bar{s}$ & 0.89581 & 0.4 \\
\hline
\end{tabular}

technique in the charm-strange system gives total mass corrections that are larger than the equal $\beta$ calculation. This is not entirely surprising since the charm strange mesons are quite close to their OZI allowed decay channels and as such we expect a large coupling between the naive states and the continuum channels. Regardless of the scheme chosen, however, the lower mass members of a multiplet seem to get the smallest corrections. There are exceptions to this pattern in the $2 S$ multiplet, for example, the $2{ }^{1} S_{0}$ state receives a larger correction than the heavier $2^{3} S_{1}$ state. The $1^{3} D_{2}-1^{1} D_{2}$ and $1^{3} P_{1}-1^{1} P_{1}$ in the $P$ and $D$ multiplets also present the same behaviour although in these cases the difference in the mass correction is not very large and the overall behaviour is very similar to what we observed in table 4.4. In our calculations we do not need to consider mixing of the states in the loop as we are summing over them. We also do not consider the mixing in the naive states, for 
Table 5.4: Comparison of the total mass corrections of various approximations in the QPC model on the total mass corrections in the charm strange sector. All values are given in units of $\mathrm{MeV}$.

\begin{tabular}{ccccc}
\hline State & $\Delta M(1 \beta)$ & $\Delta M(3 \beta)$ & $\Delta M\left(1 \beta+r_{q}\right)$ & $\Delta M\left(3 \beta+r_{q}\right)$ \\
\hline $1^{1} S_{0}$ & -277.88 & -394.73 & -63.03 & -47.85 \\
$1^{3} S_{1}$ & -317.58 & -433.71 & -72.97 & -57.68 \\
\hline $1^{3} P_{0}$ & -297.37 & -431.05 & -72.97 & -57.68 \\
$1^{1} P_{1}$ & -386.68 & -470.32 & -103.74 & -78.72 \\
$1^{3} P_{1}$ & -386.69 & -472.66 & -103.41 & -77.96 \\
$1^{3} P_{2}$ & -399.82 & -467.04 & -108.18 & -81.90 \\
\hline $2^{1} S_{0}$ & -287.59 & -373.02 & -82.46 & -72.32 \\
$2^{3} S_{1}$ & -273.79 & -353.79 & -72.78 & -65.35 \\
\hline $1^{3} D_{1}$ & -258.97 & -342.36 & -67.86 & 59.50 \\
$1^{1} D_{2}$ & -367.39 & -395.65 & -109.43 & -82.80 \\
$1^{3} D_{2}$ & -343.52 & -389.04 & -97.15 & -76.85 \\
$1^{3} D_{3}$ & -447.29 & -437.71 & -143.06 & -104.68 \\
\hline
\end{tabular}

example between $1^{3} P_{1}-1^{1} P_{1}$, since the total mass corrections that the two states receive are very close to each other and therefore we take, as an approximation, the physical states to be un-mixed.

We can also consider the Pennington Subtraction of Eq. 5.4. For our calculations we set it to be the physical mass of the $D_{s}^{+}$, the ground state of the charm strange sector where one would expect the coupled channel contributions to be minimal. In table 5.5 we see that while the total mass corrections decrease greatly, the general behaviour observed in table 5.4 remains the same. This is an issue as one would expect that if the coupled channel method is to explain anomalies such as the $D_{s}(2317)$, it should preferentially give larger contributions to the ${ }^{3} P_{0}$ state in order to bring the 
Table 5.5: Mass shifts from the coupled channel calculation (same as table 5.4) including the suggested rescaling of Eq. 5.4 from Ref. [83]. While the absolute value of the total mass corrections are reduced, we see that the general behaviour has stayed the same. All values are given in units of $\mathrm{MeV}$.

\begin{tabular}{ccccc}
\hline State & $\Delta M(1 \beta)$ & $\Delta M(3 \beta)$ & $\Delta M\left(1 \beta+r_{q}\right)$ & $\Delta M\left(3 \beta+r_{q}\right)$ \\
\hline $1^{1} S_{0}$ & -2.30 & -1.94 & -0.56 & -0.34 \\
$1^{3} S_{1}$ & -40.58 & -44.54 & -10.09 & -6.68 \\
\hline $1^{3} P_{0}$ & -89.36 & -132.18 & -16.76 & -20.32 \\
$1^{1} P_{1}$ & -180.78 & -184.05 & -53.15 & -36.12 \\
$1^{3} P_{1}$ & -180.81 & -183.93 & -52.79 & -35.51 \\
$1^{3} P_{2}$ & -192.19 & -192.54 & -57.16 & -39.23 \\
\hline $2^{1} S_{0}$ & -137.41 & -161.85 & -45.51 & -40.01 \\
$2^{3} S_{1}$ & -123.11 & -151.15 & -35.76 & -33.65 \\
\hline $1^{3} D_{1}$ & -116.74 & -152.46 & -30.30 & -27.40 \\
$1^{1} D_{2}$ & -224.02 & -217.43 & -71.77 & -51.59 \\
$1^{3} D_{2}$ & -200.86 & -208.99 & -59.65 & -45.40 \\
$1^{3} D_{3}$ & -302.08 & -268.83 & -104.94 & -74.28 \\
\hline
\end{tabular}


theoretical predictions in line with the experimental observations (see Fig. 5.1).

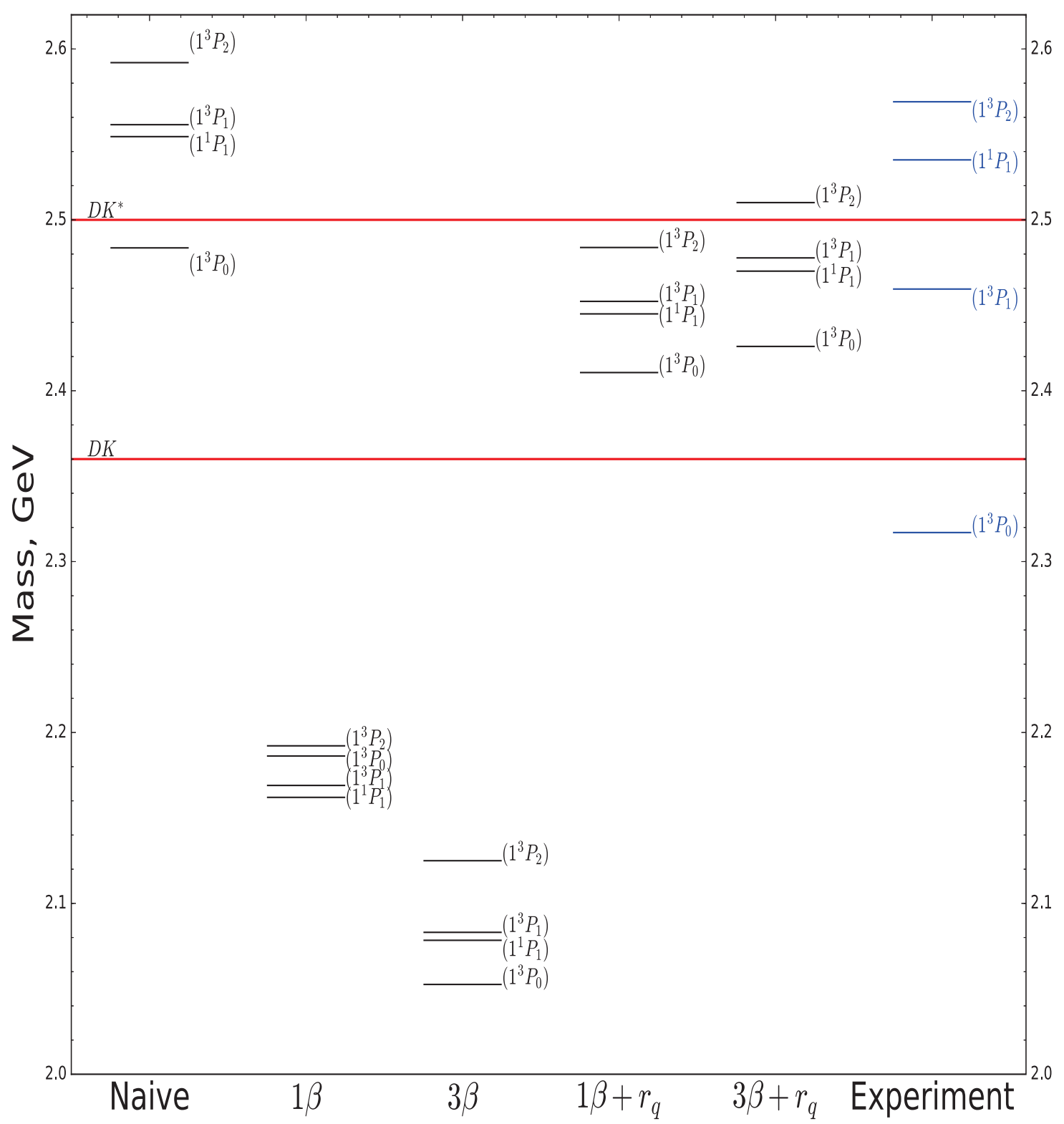

Figure 5.2: Modification of the naive charm-strange P-wave states through coupling to OZI allowed decay channels (we only show the $D K$ and $D K^{*}$ channels as others are beyond the scale of this plot). We do not use the rescaling calculation here. We show the effect of the total mass contributions to constituent quark model calculation from the different approximations of the QPC model used in this work (notation defined in chapter 4), labeled on the x-axis. 


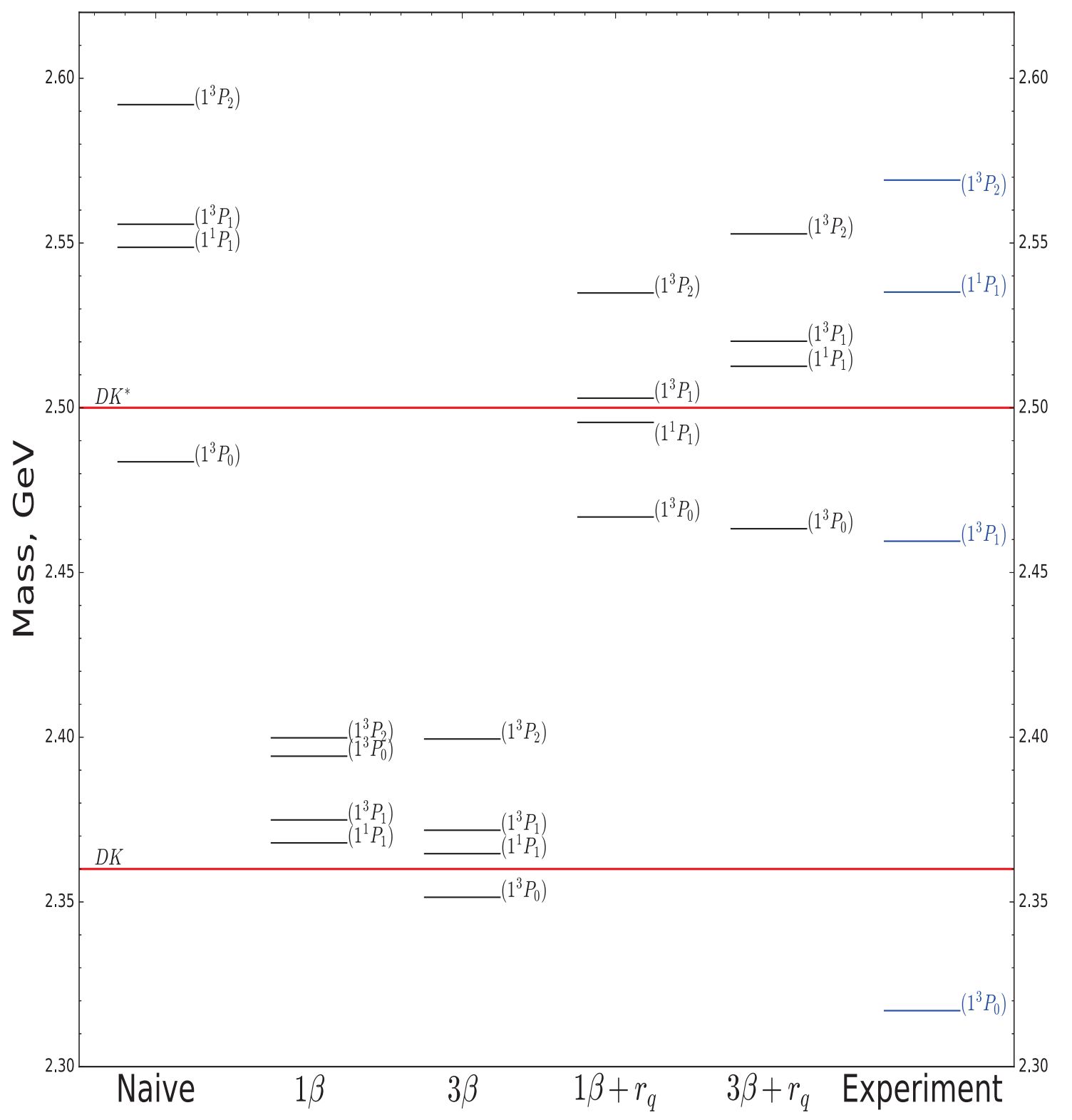

Figure 5.3: Modification of the naive charm-strange P-wave states through coupling to OZI allowed decay channels (we only show the $D K$ and $D K^{*}$ channels as others are beyond the scale of this plot). Calculations are rescaled using $m_{D^{+}}=1.96830 \mathrm{GeV}$ as the anchor mass. We show the effect of the total mass contributions to constituent quark model calculation from the different approximations of the QPC model used in this work (notation defined in chapter 4), labeled on the x-axis. 
In figures 5.2 and 5.3 we see the effect of the mass corrections of tables 5.4 and 5.5 on the P-wave states of the charm-strange system. It is clear that none of the schemes can match the experimental observations. For emphasis we reiterate that we are not necessarily interested in absolute values but rather the behaviour and the ordering of the final spectrum. The equal $\beta$ case does not match the observed spectrum at all, with its $1^{3} P_{0}$ state ending up as the second heaviest member of the multiplet. In the other three calculations, while $1^{3} P_{0}$ ends up in the right order in position as compared to other members (i.e.in the ground state), the $1^{3} P_{1}$ level does not. An interesting point to raise is the behaviour of the mass corrections in table 5.5 for the $\mathrm{P}$-waves as we move from an equal $\beta+r_{q}$ to unequal $\beta+r_{q}$. While contributions to ${ }^{1,3,3} P_{1,1,2}$ states decrease in magnitude, that of the $1^{3} P_{0}$ state increases. Since such behaviour is observed only after we apply the rescaling calculation and include a more realistic set of the wavefunctions involved, it is possible to argue that for a thorough study of the coupled channel effects and whether they can fix observed issues in the charm strange sector, one must perform the calculation in the iterative way mentioned before and use more realistic wave functions. This could mean that rather than using harmonic oscillator wave functions as faithful approximations, we could use fitted functions to the exact numerical results acquired from solving the naive potential model and continue with the mass correction calculations until the corrections converge.

We could also consider the general shape of the mass correction functions for the charm-strange P-wave states as a function of mass. In Fig. 5.4 we see that there are regions of the naive mass where the $1^{3} P_{0}$ state receives the largest corrections and if the naive masses (shown as vertical lines) were lower than their predicted values, the overall correction could in principle account for the low mass of the $D_{s}(2317)$. The issue with the $D_{s}(2460)$, however, persists as the corrections to $1^{1} P_{1}$ and $1^{3} P_{1}$ closely 


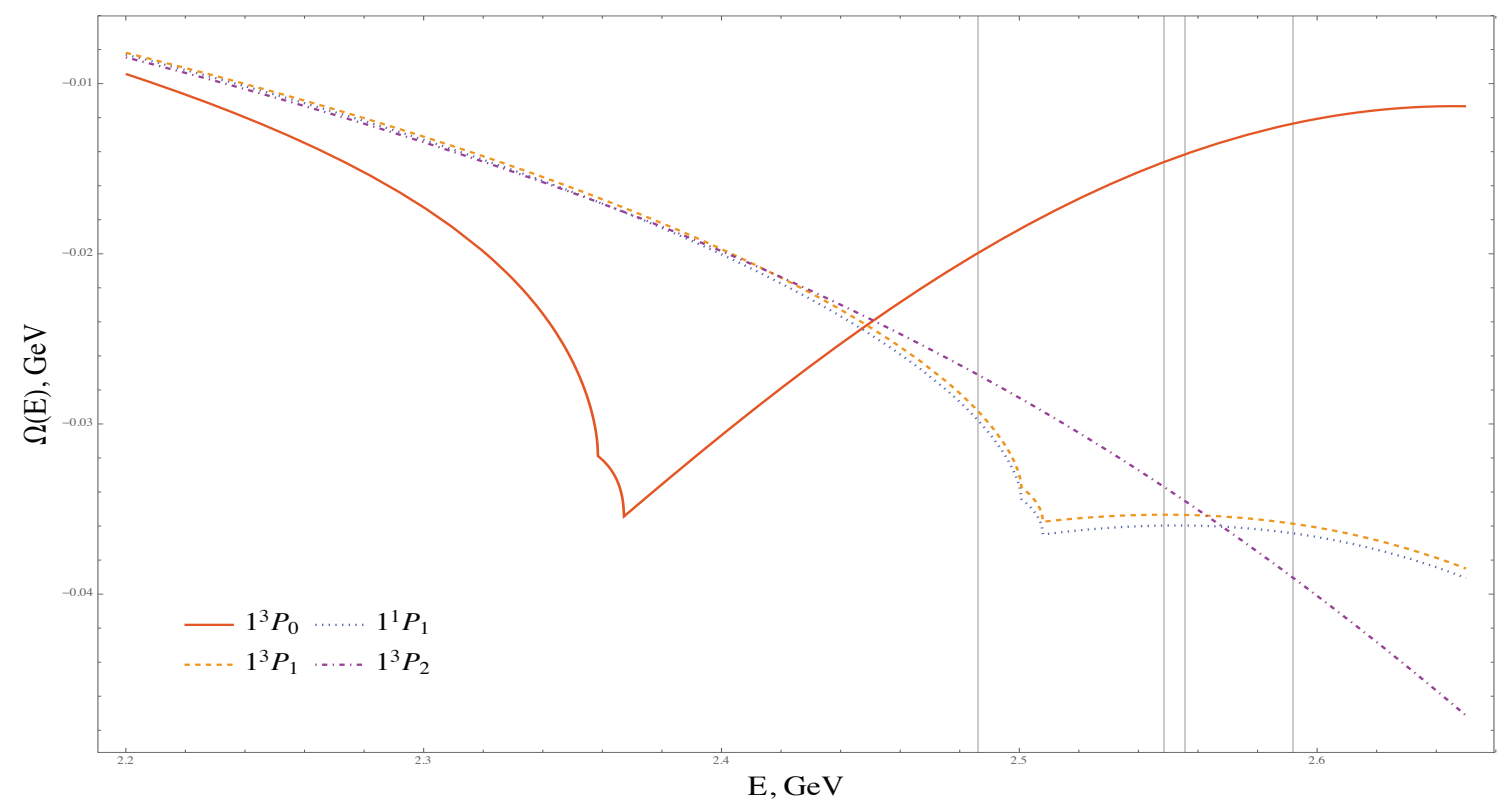

Figure 5.4: Mass corrections to the charm strange $1 P$ multiplet as a function of the mass of the state. The four vertical lines are the naive masses of the multiplet. The plot includes the effects of a quark radius factor but no rescaling calculation. It also includes the contributions of all $D K, D^{*} K, D K^{*}$ and $D^{*} K^{*}$ channels.

track each other. This remains the case regardless of the scheme used.

It should be stressed that we only include $1 S+1 S$ states in the loop and adding more radial or orbital excitations of kaons could perhaps alleviate some of these issues. One other possible explanation of our results could be that while coupled channel corrections do play a role, they are not the only possibilities for the anomolous mass of the $D_{s}(2317)$ and the $D_{s}(2460)$ and the disagreement between the predictions of the quark model and the masses of these states is due to coupled channel effects plus other physics which is discussed in section 6.1.3 of Ref. [8]. 


\section{Chapter 6}

\section{Conclusion}

This work was started with the goal of applying a simple extension to the quark model to determine its viability to explain some observed exotic states. However, it has morphed into a review of calculations performed using this framework and the choice of the model for the strong interaction vertex, the Quark Pair Creation Model, in different mesonic spectra. We saw that of the various approximations made in these computations, the $3 \beta+r_{q}$ scheme seems to be the best approach to handle loop mass corrections.

The results of our calculations for excited mesons in the loop indicate that there is more that needs to be done to understand their contributions to the total loop mass correction and that one cannot simply truncate the series at the $1 S+1 S$ components

of the continuum spectra. This, in our view, raises a serious question regarding the viability of the previously published calculations in the coupled channel model. Others have also agreed with this point [67]. We did not include the excited loop calculations with the Pennington subtraction scheme and there is a chance that such a scheme could regulate the excited meson contributions. This is a priority to be examined in future work.

As for the ability of the coupled channel framework to explain mass anomalies, 
we applied the calculation to the $D_{s}(2317)$ and other members of $1 P$ charm-strange multiplet, assuming that for now we can ignore excited meson contributions. The computations for such corrections are quite often applied to a single state within the $1 P$ multiplet. That is to say that if the goal of the calculation is to understand coupled channel effects on a given state, the only contributions calculated are to that specific state and the other members of the multiplet are not included. In our calculation, we considered not only other $1 P$ states, but also the $1 S, 2 S$ and $1 D$ multiplets of the charm strange spectrum. Our observation is that while in three of the four schemes (unequal $\beta$ with and without a form factor, equal $\beta$ with a form factor) the $1^{3} P_{0}$ does remain the lowest lying state, the other members of the multiplet receive larger corrections and move with it. Given that we wish the $D_{s}(2317)$ state to be particularly affected by its coupling to the $D K$ threshold, our observation indicates that perhaps a more detailed view of the coupled channel calculation is necessary to better understand whether a coupled channel approach can in fact account for the anomalous mass of this state. It should also be noted that given the fact that all members of the multiplet receive roughly the same amount of correction, performing the calculation for just one state of the multiplet is not, in our view, a sensible approximation.

Moving on, as discussed in chapter 2, Ackleh et al. in Ref. [36] found that the one gluon exchange potential had a significant contribution to the ${ }^{3} P_{0} \rightarrow{ }^{1} S_{0}+{ }^{1} S_{0}$. Assuming that excited meson contributions are under control in that model, then the coupled channel model can be used with both the QPC and the One Gluon Exchange potential with the OGE providing an extra mass correction to the $1^{3} P_{0}$ state relative to the other members of the multiplet.

There are further avenues that can be explored within this framework while staying with the QPC. In our calculations, we took the $\gamma_{0}$ factor, which sets the strength of 
the pair creation vertex, to be a constant. One could introduce a scale dependence to this parameter to perhaps better take into account, for systems with a heavy quark at least, the true vertex of strong decay [84]. This could be part of a future work.

Wave function approximation is also another way of improving the calculations. We consistently saw that an unequal $\beta$ calculation gave different results compared to the equal $\beta$ case and that this matters especially for states near threshold (as we saw for the $D_{s}(1 P)$ system). A natural next step would be calculations of the same mass corrections but using fitted wavefunctions of the exact numerical solutions of the Schrödinger equation. This could be done by either using a Harmonic Oscillator basis series or the Gaussian Expansion Method. The wave function is quite an important factor given that the momentum overlap integrals involve three wave functions which are evaluated at different points as the integral proceeds. Therefore it matters if the details of the exact numerical wave function corresponding to the masses that are used in the calculation are being taken into account in a detailed way. In short, our work seems to validate the conclusions of Ref. [47].

Another issue which we did not include in our calculations (and only briefly touched on) is that of mixing. In a mesonic system, states with equal $n$ and $J$ quantum numbers can mix. While this mixing is not important within the loops since we are summing over all possible loop configurations, it is quite important for naive states. A complete coupled channel calculation must construct a matrix of naive states, with diagonal terms as basis states and off-diagonal elements that connect and mix the basis states. Including the imaginary part of the mass correction function and diagonalizing the matrix should then give the observable physical mass and the corresponding width, simultaneously. This method could also predict mixing angles for the equal $n J$ states, which can be compared to experiment.

One major factor, of course, is the transition operator itself. As was mentioned in 
chapter 2, the QPC operator is one model of the strong vertex and there are others. The model closest to the fundamental mechanism for strong decay is the flux tube breaking model (FTB) $[85,86]$ and it has been shown before that the QPC model is an approximation of it [87]. One could use the flux tube breaking model instead of the QPC in order to study both the decay mechanisms as well as the mass shifts in mesonic mass spectra. As discussed in chapter 2 there are many more models of strong decay and any one of them could potentially be studied for their effect on mass corrections. The one with immediate interest is the Cornell Model for strong decays.

We conclude by remarking that our understanding of the coupled channel technique needs to be further refined before we can say conclusively whether it does in fact account for anomalies in various spectra. What we have found is that assumptions for meson wave function parameters $\beta$ and truncation of the loop mass correction series to only $1 S+1 S$ do not seem justifiable in light of our calculations here, casting doubt on the reliability of many published results in the literature. Given that the mathematical foundation of the process is sound and it is a perfectly acceptable quantum mechanical effect which expands the Fock space representation of our system from a simple $q \bar{q}$ to $|q \bar{q}\rangle+\left|M M^{\prime}\right\rangle$, the calculation should in principle work. For suspected low lying exotic states in any given spectra, one can reasonably expect the coupled channel mechanisms to be behind the discrepancies of quark model calculations and experimental observations. However, this has yet to be demonstrated. 


\section{Appendix A}

\section{Angular Momentum, Spin, Flavour and Colour Calculations}

The following is reproduced from Ref. [46], with only minor modification, and is presented here solely for completeness.

\section{A.1 Clebsch-Gordan Coefficients and the Wigner $n j$ Symbols}

An angular momentum state, be it spin, orbital angular momentum, or a vector sum of some combination of these, is represented (in an $\mathrm{SU}(2)$ algebra) by the usual quantum

numbers $|j m\rangle$ where the magnitude of the total angular momentum is $\sqrt{j(j+1)}$ and the $z$-component is $m$. To combine two angular momentum states we use the ClebschGordan coefficients, defined by

$$
\left|j_{1} m_{1}\right\rangle\left|j_{2} m_{2}\right\rangle \equiv \sum_{j=\left|j_{1}-j_{2}\right|}^{j_{1}+j_{2}}\left\langle j_{1} m_{1} j_{2} m_{2} \mid J M\right\rangle|J M\rangle
$$

where $\left\langle j_{1} m_{1} j_{2} m_{2} \mid J M\right\rangle$ is the Clebsch-Gordan coefficient and $M=m_{1}+m_{2}$. 
Appendix D. Evaluating the Colour, Flavour and Spin Overlaps...

The Wigner $3 j$ symbol is related to the Clebsch-Gordan coefficient by

$$
\left(\begin{array}{ccc}
j_{1} & j_{2} & J \\
m_{1} & m_{2} & -M
\end{array}\right) \equiv \frac{(-1)^{j_{1}-j_{2}+M}}{\sqrt{2 J+1}}\left\langle j_{1} m_{1} j_{2} m_{2} \mid J M\right\rangle .
$$

The Wigner $6 j$ symbol

$$
\left\{\begin{array}{lll}
j_{1} & j_{2} & j_{3} \\
J_{1} & J_{2} & J_{3}
\end{array}\right\}
$$

is used to combine three angular momentum states, and the Wigner $9 j$ symbol

$$
\left\{\begin{array}{ccc}
j_{1} & j_{2} & J_{12} \\
j_{3} & j_{4} & J_{34} \\
J_{13} & J_{24} & J
\end{array}\right\}
$$

is used to combine four angular momentum states. We will not define these here, though the $9 j$ symbol is defined in Appendix A.2.3. For more information, see for example Reference [88].

\section{A.2 Evaluating the Colour, Flavour and Spin Overlaps for WPC Model of Meson Decay}

In the ${ }^{3} P_{0}$ and flux-tube breaking models of meson decay, the overlaps of the colour, flavour, spin and space wavefunctions of the mesons and the created pair must be calculated. The overlap of the space wavefunctions is accomplished by the integral of Eq. 2.23. The other overlaps are discussed below. 
Appendix D. Evaluating the Colour, Flavour and Spin Overlaps...

\section{A.2.1 Colour Overlap}

The calculation of the colour overlap is particularly simple, because the mesons and

the created pair are all colour singlets, with the wavefunction $\omega=\frac{1}{\sqrt{3}}(R \bar{R}+G \bar{G}+B \bar{B})$ (using the arbitrary three colours Red, Green, Blue).

This may be represented in matrix form (this may seem excessive now, but will be useful for the flavour overlaps as well) by

$$
\omega=\frac{1}{\sqrt{3}}\left(\begin{array}{ccc}
1 & 0 & 0 \\
0 & 1 & 0 \\
0 & 0 & 1
\end{array}\right)
$$

where the rows indicate a quark of colour $R, G, B$, and the columns indicate antiquarks of color $\bar{R}, \bar{G}, \bar{B}$.

Consider a simple case with only one meson on each side, $\left\langle\omega_{A}^{12} \mid \omega_{B}^{12}\right\rangle$. The superscripts indicate that the quark of $A$ (labelled 1 ) is also the quark of $B$, and similarly for the antiquark (labelled 2). A particular colour combination (e.g. $R \bar{R}$ ) will only contribute to the overlap if it is found on both sides of the bra-ket, and the resulting term is just the product of the coefficients in the matrices for that colour combination. Then the total result is just the sum of these terms over the possible colours,

$$
\left\langle\omega_{A}^{12} \mid \omega_{B}^{12}\right\rangle=\sum_{c_{1}, c_{2}}\left(\omega_{A}\right)_{c_{1} c_{2}}\left(\omega_{B}\right)_{c_{1} c_{2}}=\sum_{c_{1}, c_{2}}\left(\omega_{A}\right)_{c_{1} c_{2}}\left(\omega_{B}^{T}\right)_{c_{2} c_{1}}=\operatorname{Tr}\left[\omega_{A} \omega_{B}^{T}\right]
$$

where $c_{1}\left(c_{2}\right)$ runs over the quark (antiquark) colours.

This is easily expanded to the case where there are two mesons on each side (e.g. 
Appendix D. Evaluating the Colour, Flavour and Spin Overlaps...

for the first diagram of Figure 2.5):

$$
\begin{aligned}
\left\langle\omega_{B}^{14} \omega_{C}^{32} \mid \omega_{A}^{12} \omega_{0}^{34}\right\rangle & =\sum_{c_{1}, c_{2}, c_{3}, c_{4}}\left(\omega_{B}\right)_{c_{1} c_{4}}\left(\omega_{C}\right)_{c_{3} c_{2}}\left(\omega_{A}\right)_{c_{1} c_{2}}\left(\omega_{0}\right)_{c_{3} c_{4}} \\
& =\sum_{c_{1}, c_{2}, c_{3}, c_{4}}\left(\omega_{A}^{T}\right)_{c_{2} c_{1}}\left(\omega_{B}\right)_{c_{1} c_{4}}\left(\omega_{0}^{T}\right)_{c_{4} c_{3}}\left(\omega_{C}\right)_{c_{3} c_{2}}=\operatorname{Tr}\left[\omega_{A}^{T} \omega_{B} \omega_{0}^{T} \omega_{C}\right] .
\end{aligned}
$$

For the case where everything is a colour singlet, the results are

$$
\left\langle\omega_{B}^{14} \omega_{C}^{32} \mid \omega_{A}^{12} \omega_{0}^{34}\right\rangle=\left\langle\omega_{B}^{32} \omega_{C}^{14} \mid \omega_{A}^{12} \omega_{0}^{34}\right\rangle=\frac{1}{3} .
$$

\section{A.2.2 Flavour Overlap}

The overlap of the flavour wavefunctions of the mesons and the created pair can be calculated using the matrix notation introduced in Appendix A.2.1. The flavour of a meson may be represented by a matrix where the rows indicate a quark of flavour $u$, $d, s, \ldots$ and the columns indicate antiquarks of color $\bar{u}, \bar{d}, \bar{s}, \ldots$ For example, consider the $\pi^{0}$,

$$
\phi_{\pi^{0}}=\frac{1}{\sqrt{2}}(u \bar{u}-d \bar{d})=\frac{1}{\sqrt{2}}\left(\begin{array}{ccc}
1 & 0 & 0 \\
0 & -1 & 0 \\
0 & 0 & 0
\end{array}\right)
$$

and the $K^{+}$,

$$
\phi_{K^{+}}=-u \bar{s}=\left(\begin{array}{ccc}
0 & 0 & -1 \\
0 & 0 & 0 \\
0 & 0 & 0
\end{array}\right) .
$$

The ${ }^{3} P_{0}$ and flux-tube breaking models assume that the pair is created in an 
Appendix D. Evaluating the Colour, Flavour and Spin Overlaps...

$\mathrm{SU}(\mathrm{N})$ flavour singlet; we take $\mathrm{N}=3$, since we are not concerned with any mesons involving $\mathrm{c}, \mathrm{b}$ or t quarks:

$$
\phi_{0}=\frac{1}{\sqrt{3}}(u \bar{u}+d \bar{d}+s \bar{s})=\frac{1}{\sqrt{3}}\left(\begin{array}{ccc}
1 & 0 & 0 \\
0 & 1 & 0 \\
0 & 0 & 1
\end{array}\right) .
$$

Taking $\mathrm{N}$ to be some other number would change the value of $\gamma\left(\gamma_{0}\right)$ needed to fit the data (because of the normalization in Eq. A.9), but would not change the calculated decay widths.

The overlap is given by expressions similar to Eq. A.5:

$$
\begin{aligned}
& \left\langle\phi_{B}^{14} \phi_{C}^{32} \mid \phi_{A}^{12} \phi_{0}^{34}\right\rangle=\operatorname{Tr}\left[\phi_{A}^{T} \phi_{B} \phi_{0}^{T} \phi_{C}\right]=\frac{1}{\sqrt{3}} \operatorname{Tr}\left[\phi_{A}^{T} \phi_{B} \phi_{C}\right] \\
& \left\langle\phi_{B}^{32} \phi_{C}^{14} \mid \phi_{A}^{12} \phi_{0}^{34}\right\rangle=\operatorname{Tr}\left[\phi_{A}^{T} \phi_{C} \phi_{0}^{T} \phi_{B}\right]=\frac{1}{\sqrt{3}} \operatorname{Tr}\left[\phi_{A}^{T} \phi_{C} \phi_{B}\right] .
\end{aligned}
$$

In many cases only one of these overlaps will be non-zero.

As an example, consider the flavour overlaps in the decay $K^{*}(892)^{+} \rightarrow K^{+} \pi^{0}$; $\phi_{K^{*}(892)^{+}}=\phi_{K^{+}}$, giving us

$$
\begin{aligned}
\left\langle\phi_{B}^{14} \phi_{C}^{32} \mid \phi_{A}^{12} \phi_{0}^{34}\right\rangle & =0 \\
\left\langle\phi_{B}^{32} \phi_{C}^{14} \mid \phi_{A}^{12} \phi_{0}^{34}\right\rangle & =\frac{1}{\sqrt{6}} .
\end{aligned}
$$


Appendix D. Evaluating the Colour, Flavour and Spin Overlaps...

\section{A.2.3 Spin Overlap}

Since quarks have spin $\frac{1}{2}$, their spins may only combine to give 0 or 1 in a meson. They combine in the usual singlet and triplet states (given as e.g. $\left|S_{A} M_{S_{A}}\right\rangle$ ):

$$
\begin{aligned}
|00\rangle & =\frac{1}{\sqrt{2}}\left[\left|\frac{1}{2} \frac{1}{2}\right\rangle\left|\frac{1}{2}-\frac{1}{2}\right\rangle-\left|\frac{1}{2}-\frac{1}{2}\right\rangle\left|\frac{1}{2} \frac{1}{2}\right\rangle\right] \\
|11\rangle & =\left|\frac{1}{2} \frac{1}{2}\right\rangle\left|\frac{1}{2} \frac{1}{2}\right\rangle \\
|10\rangle & =\frac{1}{\sqrt{2}}\left[\left|\frac{1}{2} \frac{1}{2}\right\rangle\left|\frac{1}{2}-\frac{1}{2}\right\rangle+\left|\frac{1}{2}-\frac{1}{2}\right\rangle\left|\frac{1}{2} \frac{1}{2}\right\rangle\right] \\
|1-1\rangle & =\left|\frac{1}{2}-\frac{1}{2}\right\rangle\left|\frac{1}{2}-\frac{1}{2}\right\rangle
\end{aligned}
$$

The overlap of the spin wavefunctions of the mesons and the created pair can be calculated by angular momentum algebra. It can be expanded in a sum over possible total spins (e.g. for the first diagram of Figure 2.5)

$$
\begin{aligned}
& \left\langle\chi_{S_{B} M_{S_{B}}}^{14} \chi_{S_{C} M_{S_{C}}}^{32} \mid \chi_{S_{A} M_{S_{A}}}^{12} \chi_{1-m}^{34}\right\rangle= \\
& \quad \sum_{S, M_{S}}\left\langle\left(j_{1} j_{4}\right) S_{B},\left(j_{3} j_{2}\right) S_{C} ; S M_{S} \mid\left(j_{1} j_{2}\right) S_{A},\left(j_{3} j_{4}\right) 1 ; S M_{S}\right\rangle \\
& \quad \times\left\langle S_{B} M_{S_{B}} S_{C} M_{S_{C}} \mid S M_{S}\right\rangle\left\langle S_{A} M_{S_{A}} 1-m \mid S M_{S}\right\rangle
\end{aligned}
$$

where the $j_{i}$ 's are all $\frac{1}{2}$, but are necessarily written this way to show the connection between the left and right sides of the first factor. The first factor is a transformation between two different bases for combining four angular momenta, and is independent of $M_{S}$. On the left side, $j_{1}$ and $j_{4}$ are added together to obtain $S_{B}$, which is added to $S_{C}$ to obtain $S$ - a similar procedure is carried out on the right side. The $9 j$ symbol 
Appendix D. Evaluating the Colour, Flavour and Spin Overlaps...

is defined in terms of this transformation,

$$
\begin{aligned}
& \left\langle\left(j_{1} j_{4}\right) J_{14},\left(j_{3} j_{2}\right) J_{32} ; J M \mid\left(j_{1} j_{2}\right) J_{12},\left(j_{3} j_{4}\right) J_{34} ; J M\right\rangle= \\
& \quad \times\left\{\begin{array}{ccc}
j_{1} & j_{2} & J_{12} \\
j_{4} & j_{3} & J_{34} \\
J_{14} & J_{32} & J
\end{array}\right\},
\end{aligned}
$$

which allows us to write the spin overlap in terms of a $9 j$ symbol:

$$
\begin{aligned}
& \left\langle\chi_{S_{B} M_{S_{B}}}^{14} \chi_{S_{C} M_{S_{C}}}^{32} \mid \chi_{S_{A} M_{S_{A}}}^{12} \chi_{1-m}^{34}\right\rangle= \\
& (-1)^{S_{C}+1} \sqrt{3\left(2 S_{A}+1\right)\left(2 S_{B}+1\right)\left(2 S_{C}+1\right)} \sum_{S, M_{S}}\left\langle S_{B} M_{S_{B}} S_{C} M_{S_{C}} \mid S M_{S}\right\rangle \\
& \times\left\langle S_{A} M_{S_{A}} 1-m \mid S M_{S}\right\rangle\left\{\begin{array}{ccc}
\frac{1}{2} & \frac{1}{2} & S_{A} \\
\frac{1}{2} & \frac{1}{2} & 1 \\
S_{B} & S_{C} & S
\end{array}\right\} .
\end{aligned}
$$

Using an alternative definition of the $9 j$ symbol that couples the quarks differently, we can get a similar expression for the spin overlap for the second diagram of Figure 2.5 which satisfies

$$
\left\langle\chi_{S_{B} M_{S_{B}}}^{32} \chi_{S_{C} M_{S_{C}}}^{14} \mid \chi_{S_{A} M_{S_{A}}}^{12} \chi_{1-m}^{34}\right\rangle=(-1)^{1+S_{A}+S_{B}+S_{C}}\left\langle\chi_{S_{B} M_{S_{B}}}^{14} \chi_{S_{C} M_{S_{C}}}^{32} \mid \chi_{S_{A} M_{S_{A}}}^{12} \chi_{1-m}^{34}\right\rangle
$$

Eq. A.17 was used to simplify Eq. 2.21. 


\section{Appendix B}

\section{Field Theory Conventions}

The following is reproduced from Ref. [46], with only minor modification, and is presented here solely for completeness.

We use the following field theory conventions in this work:

For the spinor normalizations,

$$
\begin{aligned}
& u^{(\alpha) \dagger}(p) u^{(\beta)}(p)=v^{(\alpha) \dagger}(p) v^{(\beta)}(p)=\frac{E}{m} \delta_{\alpha \beta} \\
& u^{(\alpha) \dagger}(p) v^{(\beta)}(\bar{p})=v^{(\alpha) \dagger}(p) u^{(\beta)}(\bar{p})=0,
\end{aligned}
$$

where if $p=(E, \vec{p})$, then $\bar{p} \equiv(E,-\vec{p})$.

We define the spinor field

$$
\Psi(x)=\sum_{\alpha} \int \frac{\mathrm{d}^{3} \vec{p}}{(2 \pi)^{\frac{3}{2}}} \sqrt{\frac{m}{E}}\left[b_{\alpha}(p) u^{(\alpha)}(p) e^{-i p \cdot x}+d_{\alpha}^{\dagger}(p) v^{(\alpha)}(p) e^{i p \cdot x}\right]
$$


and use the anticommutators

$$
\begin{aligned}
\left\{\Psi_{i}(t, \vec{x}), \Psi_{j}^{\dagger}\left(t, \vec{x}^{\prime}\right)\right\} & =\delta^{3}\left(\vec{x}-\vec{x}^{\prime}\right) \delta_{i j} \\
\left\{\Psi_{i}(t, \vec{x}), \Psi_{j}\left(t, \vec{x}^{\prime}\right)\right\}=\left\{\Psi_{i}^{\dagger}(t, \vec{x}), \Psi_{j}^{\dagger}\left(t, \vec{x}^{\prime}\right)\right\} & =0 \\
\left\{b_{\alpha}(p), b_{\alpha^{\prime}}^{\dagger}\left(p^{\prime}\right)\right\}=\left\{d_{\alpha}(p), d_{\alpha^{\prime}}^{\dagger}\left(p^{\prime}\right)\right\} & =\delta^{3}\left(\vec{p}-\vec{p}^{\prime}\right) \delta_{\alpha \alpha^{\prime}} \\
\left\{b_{\alpha}(p), b_{\alpha^{\prime}}\left(p^{\prime}\right)\right\}=\left\{d_{\alpha}(p), d_{\alpha^{\prime}}\left(p^{\prime}\right)\right\} & =0 \\
\left\{b_{\alpha}^{\dagger}(p), b_{\alpha^{\prime}}^{\dagger}\left(p^{\prime}\right)\right\}=\left\{d_{\alpha}^{\dagger}(p), d_{\alpha^{\prime}}^{\dagger}\left(p^{\prime}\right)\right\} & =0,
\end{aligned}
$$

which gives for the normalization of one particle states

$$
\left\langle b_{\alpha}(p) \mid b_{\alpha^{\prime}}\left(p^{\prime}\right)\right\rangle=\left\langle d_{\alpha}(p) \mid d_{\alpha^{\prime}}\left(p^{\prime}\right)\right\rangle=\delta^{3}\left(\vec{p}-\vec{p}^{\prime}\right) \delta_{\alpha \alpha^{\prime}}
$$




\section{Appendix $\mathrm{C}$}

\section{Complete Bottomonium Results}

Here we present our results for the $1 S+1 S$ loop corrections to $75 b \bar{b}$ states, comparing $1 \beta$ vs. $3 \beta$ and $1 \beta+r_{q}$ vs. $3 \beta+r_{q}$ computations. We will also present the results for mass corrections to $\Upsilon(1 S)$ with excited mesons in the loop. All numbers in this appendix are presented in units of $\mathrm{MeV}$. 
Table C.1: Loop corrections to $\Upsilon(1 S)$ including radially and orbitally excited mesons with $1 \beta$ scheme without rescaling.

\begin{tabular}{|c|c|c|c|c|c|}
\hline Loop Content & $\Delta M(M e V)$ & & & & \\
\hline $1 S+1 S$ & -474.34 & & & & \\
\hline $1 S+2 S$ & -114.92 & $4 S+4 S$ & -342.48 & & \\
\hline $1 S+3 S$ & -19.94 & $4 S+5 S$ & -109.94 & & \\
\hline $1 S+4 S$ & -2.72 & $4 S+1 P$ & -8.60 & & \\
\hline $1 S+5 S$ & -0.26 & $4 S+2 P$ & -117.32 & & \\
\hline $1 S+1 P$ & -1815.00 & $4 S+3 P$ & -904.08 & & \\
\hline $1 S+2 P$ & -89.66 & $4 S+1 D$ & -20.16 & & \\
\hline $1 S+3 P$ & -10.42 & $4 S+2 D$ & -213.16 & & \\
\hline $1 S+1 D$ & -771.10 & $4 S+1 F$ & -22.94 & & \\
\hline $1 S+2 D$ & -54.40 & $5 S+5 S$ & -298.13 & $3 P+3 P$ & -2153.78 \\
\hline $1 S+1 F$ & -227.46 & $5 S+1 P$ & -1.24 & $3 P+1 D$ & -151.72 \\
\hline $2 S+2 S$ & -458.59 & $5 S+2 P$ & 20.48 & $3 P+2 D$ & -2389.38 \\
\hline $2 S+3 S$ & & on & -20.40 & $3 P+1 F$ & -268.08 \\
\hline $2 S+3 S$ & -108.38 & $5 S+3 P$ & -157.38 & $1 D+1 D$ & -3812.13 \\
\hline $2 S+4 S$ & -30.68 & $5 S+1 D$ & -4.34 & $1 D+2 D$ & -858.44 \\
\hline $2 S+5 S$ & -6.40 & $5 S+2 D$ & -47.00 & $1 D+2 D$ & \\
\hline $2 S+1 P$ & -828.02 & $5 S+1 F$ & -3.94 & $1 D+1 F$ & -7305.66 \\
\hline $2 S+2 P$ & -1454.46 & $1 P+1 P$ & -2271.88 & $2 D+2 D$ & -3741.14 \\
\hline $2 S+3 P$ & -146.26 & $1 P+2 P$ & -553.88 & $2 D+1 F$ & -2212.46 \\
\hline $2 S+1 D$ & -1164.86 & $1 P+3 P$ & -94.52 & $1 F+1 F$ & -5577.73 \\
\hline $2 S+2 D$ & -618.18 & $1 P+1 D$ & -4574.28 & Total & -65946.30 \\
\hline $2 S+1 F$ & -779.36 & $1 P+2 D$ & -305.20 & & \\
\hline $3 S+3 S$ & -397.44 & $1 P+1 F$ & -2264.56 & & \\
\hline $3 S+4 S$ & -109.10 & $2 P+2 P$ & -2276.02 & & \\
\hline $3 S+5 S$ & -36.36 & $2 P+3 P$ & -682.32 & & \\
\hline $3 S+1 P$ & -62.96 & $2 P+1 D$ & -1748.00 & & \\
\hline $3 S+2 P$ & -929.62 & $2 P+2 D$ & -4184.66 & & \\
\hline $3 S+3 P$ & -1243.84 & $2 P+1 F$ & & & \\
\hline $3 S+1 D$ & $\begin{array}{l}-126.62 \\
-154406\end{array}$ & & & & \\
\hline $\begin{array}{l}3 S+2 D \\
3 S+1 F\end{array}$ & $\begin{array}{l}-1544.06 \\
-285.40\end{array}$ & & & & \\
\hline $3 S+1 F$ & -285.40 & & & & \\
\hline
\end{tabular}


Table C.2: Loop corrections to $\Upsilon(1 S)$ including radially and orbitally excited mesons in $1 \beta+r_{q}$ scheme without rescaling.

\begin{tabular}{|c|c|c|c|c|c|}
\hline Loop Content & $\Delta M(M e V)$ & & & & \\
\hline $1 S+1 S$ & -105.26 & & & & \\
\hline $1 S+2 S$ & -2.68 & $4 S+4 S$ & -3.13 & & \\
\hline $1 S+3 S$ & -1.07 & $4 S+5 S$ & -3.18 & & \\
\hline $1 S+4 S$ & -1.26 & $4 S+1 P$ & -4.56 & & \\
\hline $1 S+5 S$ & -0.60 & $4 S+2 P$ & -4.50 & & \\
\hline $1 S+1 P$ & -218.50 & $4 S+3 P$ & -0.12 & & \\
\hline $1 S+2 P$ & -62.04 & $4 S+4 P$ & -4.26 & & \\
\hline $1 S+3 P$ & -13.98 & $4 S+1 D$ & -0.60 & & \\
\hline $1 S+4 P$ & -2.90 & $4 S+2 D$ & -3.40 & $3 P+3 P$ & -17.21 \\
\hline $1 S+1 D$ & -41.40 & $4 S+1 F$ & 0.00 & $3 P+4 P$ & -15.68 \\
\hline $1 S+2 D$ & -19.96 & $5 S+5 S$ & -2.06 & $3 P+1 D$ & -3.92 \\
\hline $1 S+1 F$ & -5.38 & $5 S+1 P$ & -1.52 & $3 P+2 D$ & -4.82 \\
\hline $2 S+2 S$ & -10.94 & $5 S+2 P$ & -4.14 & $3 P+1 F$ & -3.66 \\
\hline $2 S+3 S$ & -4.44 & $5 S+3 P$ & -2.36 & $4 P+1 D$ & -3.48 \\
\hline $2 S+4 S$ & -0.20 & $5 S+4 P$ & -0.04 & $4 P+2 D$ & -2.20 \\
\hline $2 S+5 S$ & -0.32 & $5 S+1 D$ & 0.00 & $4 P+1 F$ & -1.16 \\
\hline $2 S+1 P$ & -3.12 & $5 S+2 D$ & -0.78 & $1 D+1 D$ & -111.50 \\
\hline $2 S+2 P$ & -24.12 & $5 S+1 F$ & -0.04 & $1 D+2 D$ & -49.22 \\
\hline $2 S+3 P$ & -23.36 & $1 P+1 P$ & -152.17 & $1 D+1 F$ & -145.90 \\
\hline $2 S+4 P$ & -10.56 & $1 P+2 P$ & -30.28 & & \\
\hline $2 S+1 D$ & -7.64 & $1 P+3 P$ & -1.60 & $2 D+2 D$ & -41.36 \\
\hline $2 S+2 D$ & -0.80 & $1 P+4 P$ & -2.02 & $2 D+1 F$ & -18.18 \\
\hline $2 S+1 F$ & -3.40 & $1 P+1 D$ & -215.54 & $1 F+1 F$ & -71.97 \\
\hline $3 S+3 S$ & -5.17 & $1 P+2 D$ & -90.20 & Total & -1808.83 \\
\hline $3 S+4 S$ & -4.15 & $1 P+1 F$ & -50.58 & & \\
\hline $3 S+5 S$ & -0.56 & $2 P+2 P$ & -36.21 & & \\
\hline $3 S+1 P$ & -8.79 & $2 P+3 P$ & -22.14 & & \\
\hline $3 S+2 P$ & -0.54 & $2 P+4 P$ & -2.24 & & \\
\hline $3 S+3 P$ & -8.94 & $2 P+1 D$ & -9.92 & & \\
\hline $3 S+4 P$ & -13.83 & $2 P+2 D$ & -54.16 & & \\
\hline $3 S+1 D$ & -4.43 & $2 P+1 F$ & -2.12 & & \\
\hline $3 S+2 D$ & -3.90 & & & & \\
\hline $3 S+1 F$ & -0.46 & & & & \\
\hline
\end{tabular}


Table C.3: $1 \beta$ vs $3 \beta$ comparison for $1 S+1 S$ loop mass corrections.

\begin{tabular}{|c|c|c|c|c|c|c|c|c|c|c|c|c|c|c|}
\hline \multirow{3}{*}{$\begin{array}{l}\text { State } \\
\qquad \Upsilon\left(1^{3} S_{1}\right)\end{array}$} & \multicolumn{14}{|c|}{$\Omega(M)(\mathrm{MeV})$} \\
\hline & \multicolumn{2}{|c|}{$B B$} & \multicolumn{2}{|c|}{$B B^{*}$} & \multicolumn{2}{|c|}{$B^{*} B^{*}$} & \multicolumn{2}{|c|}{$B_{s} B_{s}$} & \multicolumn{2}{|c|}{$B_{S} B_{S}^{*}$} & \multicolumn{2}{|c|}{$B_{s}^{*} B_{s}^{*}$} & \multicolumn{2}{|c|}{ Total } \\
\hline & -34.02 & -30.92 & -131.43 & -111.77 & -222.41 & -180.24 & -7.56 & -10.28 & -29.28 & -37.72 & -49.67 & -61.66 & -474.36 & -432.59 \\
\hline$\eta_{b}\left(1^{1} S_{0}\right)$ & 0.00 & 0.00 & -188.13 & -153.07 & -182.20 & -141.78 & 0.00 & 0.00 & -42.16 & -53.79 & -40.91 & -50.48 & -453.40 & -399.13 \\
\hline$\chi_{b 2}\left(1^{3} P_{2}\right)$ & -63.21 & -74.84 & -180.69 & -202.90 & -302.82 & -301.00 & -13.29 & -20.45 & -38.23 & -56.50 & -57.62 & -77.71 & -655.85 & -733.40 \\
\hline$\chi_{b 1}\left(1^{3} P_{1}\right)$ & 0.00 & 0.00 & -252.25 & -253.57 & -281.73 & -300.46 & 0.00 & 0.00 & -46.52 & -63.66 & -60.14 & -86.86 & -640.64 & -704.55 \\
\hline$\chi_{b 0}\left(1^{3} P_{0}\right)$ & -117.83 & -115.84 & 0.00 & 0.00 & -400.29 & -411.12 & -19.61 & -26.28 & 0.00 & 0.00 & -84.25 & -120.26 & -621.99 & -673.50 \\
\hline$h_{b}\left(1^{1} P_{1}\right)$ & 0.00 & 0.00 & -275.19 & -293.56 & -262.28 & -266.97 & 0.00 & 0.00 & -54.83 & -78.66 & -52.60 & -73.06 & -644.90 & -712.25 \\
\hline$\Upsilon\left(1^{3} D_{3}\right)$ & -79.79 & -103.08 & -200.08 & -251.85 & -343.60 & -393.02 & -15.91 & -24.29 & -40.34 & -60.84 & -57.93 & -82.36 & -737.65 & -915.44 \\
\hline$\Upsilon\left(1^{3} D_{2}\right)$ & 0.00 & 0.00 & -303.90 & -361.56 & -312.85 & -379.28 & 0.00 & 0.00 & -51.74 & -75.84 & -60.84 & -91.05 & -729.34 & -907.73 \\
\hline$\Upsilon\left(1^{3} D_{1}\right)$ & -98.06 & -117.90 & -90.31 & -101.01 & -421.67 & -516.76 & -13.89 & -20.58 & -13.02 & -18.14 & -84.26 & -128.07 & -721.21 & -902.46 \\
\hline$\Upsilon\left(1^{1} D_{2}\right)$ & 0.00 & 0.00 & -318.58 & -387.35 & -298.81 & -353.30 & 0.00 & 0.00 & -57.90 & -86.10 & -55.00 & -80.74 & -730.29 & -907.50 \\
\hline$\Upsilon\left(1^{3} F_{4}\right)$ & -87.82 & -112.69 & -203.14 & -262.71 & -364.48 & -445.19 & -16.46 & -23.01 & -38.77 & -55.38 & -54.77 & -77.55 & -765.44 & -976.53 \\
\hline$\Upsilon\left(1^{3} F_{3}\right)$ & 0.00 & 0.00 & -332.77 & -414.89 & -321.36 & -411.57 & 0.00 & 0.00 & -51.76 & -73.80 & -57.12 & -83.59 & -763.02 & -983.85 \\
\hline$\Upsilon\left(1^{3} F_{2}\right)$ & -103.56 & -128.19 & -122.44 & -146.83 & -427.59 & -558.59 & -12.45 & -18.02 & -15.27 & -21.47 & -80.28 & -119.52 & -761.59 & -992.61 \\
\hline$\Upsilon\left(1^{1} F_{3}\right)$ & 0.00 & 0.00 & -340.47 & -429.19 & -312.70 & -394.42 & 0.00 & 0.00 & -56.20 & -80.36 & -52.82 & -76.62 & -762.19 & -980.59 \\
\hline$\Upsilon\left(1^{3} G_{5}\right)$ & -91.66 & -108.71 & -198.51 & -247.65 & -381.78 & -465.07 & -15.78 & -19.08 & -35.33 & -45.30 & -50.08 & -67.00 & -773.15 & -952.81 \\
\hline$\Upsilon\left(1^{3} G_{4}\right)$ & 0.00 & 0.00 & -358.54 & -429.18 & -321.71 & -409.73 & 0.00 & 0.00 & -49.05 & -63.68 & -51.47 & -70.18 & -780.77 & -972.77 \\
\hline$\Upsilon\left(1^{3} G_{3}\right)$ & -123.14 & -136.66 & -150.87 & -173.93 & -419.38 & -547.38 & -11.60 & -14.97 & -15.60 & -20.43 & -72.95 & -100.87 & -793.54 & -994.25 \\
\hline$\Upsilon\left(1^{1} G_{4}\right)$ & 0.00 & 0.00 & -359.22 & -434.12 & -318.22 & -400.59 & 0.00 & 0.00 & -52.15 & -67.63 & -48.40 & -65.76 & -778.00 & -968.10 \\
\hline$\Upsilon\left(1^{3} H_{6}\right)$ & -98.85 & -106.90 & -196.08 & -238.35 & -439.15 & -485.58 & -14.51 & -17.28 & -31.18 & -40.61 & -45.12 & -60.22 & -824.90 & -948.93 \\
\hline$\Upsilon\left(1^{3} H_{5}\right)$ & 0.00 & 0.00 & -443.26 & -453.95 & -337.90 & -411.58 & 0.00 & 0.00 & -45.31 & -57.41 & -45.33 & -62.83 & -871.80 & -985.77 \\
\hline$\Upsilon\left(1^{3} H_{4}\right)$ & -167.81 & -155.80 & -226.31 & -205.95 & -417.76 & -538.53 & -11.26 & -12.89 & -15.47 & -18.85 & -64.34 & -90.58 & -902.95 & -1022.60 \\
\hline$\Upsilon\left(2^{3} S_{1}\right)$ & -37.49 & -57.24 & -142.13 & -207.93 & -236.45 & -335.81 & -7.71 & -13.31 & -29.51 & -49.74 & -49.52 & -82.46 & -502.81 & -746.49 \\
\hline
\end{tabular}




\begin{tabular}{|c|c|c|c|c|c|c|c|c|c|c|c|c|c|c|}
\hline$\eta_{b}\left(2^{1} S_{0}\right)$ & 0.00 & 0.00 & -206.54 & -302.85 & -196.68 & -279.26 & 0.00 & 0.00 & -43.18 & -74.44 & -41.45 & -70.44 & -487.85 & -726.99 \\
\hline$\chi_{b 2}\left(2^{3} P_{2}\right)$ & -55.32 & -78.43 & -155.14 & -218.46 & -260.39 & -368.66 & -11.21 & -16.20 & -31.95 & -46.47 & -48.34 & -73.02 & -562.34 & -801.24 \\
\hline$\chi_{b 1}\left(2^{3} P_{1}\right)$ & 0.00 & 0.00 & -222.23 & -321.23 & -238.31 & -340.15 & 0.00 & 0.00 & -39.60 & -60.87 & -50.03 & -75.37 & -550.17 & -797.61 \\
\hline$\chi_{b 0}\left(2^{3} P_{0}\right)$ & -108.27 & -163.96 & 0.00 & 0.00 & -340.48 & -493.18 & -17.18 & -27.97 & 0.00 & 0.00 & -70.52 & -109.29 & -536.44 & -794.40 \\
\hline$h_{b}\left(2^{1} P_{1}\right)$ & 0.00 & 0.00 & -239.11 & -342.13 & -223.89 & -319.16 & 0.00 & 0.00 & -46.21 & -69.26 & -43.98 & -66.71 & -553.19 & -797.26 \\
\hline$\Upsilon\left(2^{3} D_{3}\right)$ & -68.78 & -85.41 & -168.12 & -213.62 & -286.50 & -374.24 & -13.25 & -16.26 & -33.37 & -41.97 & -46.45 & -61.85 & -616.48 & -793.35 \\
\hline$\Upsilon\left(2^{3} D_{2}\right)$ & 0.00 & 0.00 & -259.91 & -336.75 & -258.59 & -340.52 & 0.00 & 0.00 & -41.97 & -54.82 & -49.80 & -65.86 & -610.27 & -797.94 \\
\hline$\Upsilon\left(2^{3} D_{1}\right)$ & -89.04 & -116.85 & -78.12 & -103.01 & -347.79 & -462.38 & -11.16 & -15.00 & -10.31 & -14.07 & -69.24 & -92.47 & -605.65 & -803.78 \\
\hline$\Upsilon\left(2^{1} D_{2}\right)$ & 0.00 & 0.00 & -270.66 & -348.91 & -247.71 & -325.79 & 0.00 & 0.00 & -47.35 & -61.08 & -44.67 & -59.37 & -610.38 & -795.15 \\
\hline$\Upsilon\left(2^{3} F_{4}\right)$ & -87.78 & -91.33 & -184.28 & -207.95 & -328.78 & -395.49 & -14.26 & -15.20 & -33.40 & -37.09 & -44.42 & -52.46 & -692.93 & -799.52 \\
\hline$\Upsilon\left(2^{3} F_{3}\right)$ & 0.00 & 0.00 & -341.71 & -395.41 & -282.15 & -340.95 & 0.00 & 0.00 & -42.95 & -48.84 & -48.17 & -56.79 & -714.99 & -842.00 \\
\hline$\Upsilon\left(2^{3} F_{2}\right)$ & -91.75 & -129.83 & -135.60 & -159.29 & -371.60 & -451.89 & -10.03 & -11.40 & -12.00 & -14.06 & -68.47 & -81.16 & -689.45 & -847.62 \\
\hline$\Upsilon\left(2^{1} F_{3}\right)$ & 0.00 & 0.00 & -338.56 & -389.98 & -276.66 & -334.34 & 0.00 & 0.00 & -47.19 & -53.36 & -44.05 & -52.03 & -706.46 & -829.71 \\
\hline$\Upsilon\left(2^{3} G_{5}\right)$ & -68.55 & -80.30 & -176.99 & -200.15 & -298.22 & -332.36 & -14.64 & -13.52 & -32.31 & -31.95 & -42.44 & -44.73 & -633.15 & -703.00 \\
\hline$\Upsilon\left(2^{3} G_{4}\right)$ & 0.00 & 0.00 & -307.44 & -260.25 & -284.60 & -321.44 & 0.00 & 0.00 & -43.29 & -43.21 & -45.66 & -48.51 & -681.00 & -673.42 \\
\hline$\Upsilon\left(2^{3} G_{3}\right)$ & -125.17 & -57.63 & -123.37 & -74.84 & -392.10 & -444.94 & -10.96 & -9.90 & -13.04 & -13.16 & -65.78 & -70.21 & -730.42 & -670.68 \\
\hline$\Upsilon\left(2^{1} G_{4}\right)$ & 0.00 & 0.00 & -310.67 & -281.85 & -272.53 & -307.95 & 0.00 & 0.00 & -46.43 & -46.29 & -42.48 & -45.14 & -672.11 & -681.22 \\
\hline$\Upsilon\left(3^{3} S_{1}\right)$ & -35.98 & -52.63 & -133.31 & -196.89 & -217.83 & -326.70 & -7.05 & -9.74 & -26.78 & -37.76 & -44.64 & -64.77 & -465.58 & -688.49 \\
\hline$\eta_{b}\left(3^{1} S_{0}\right)$ & 0.00 & 0.00 & -194.21 & -297.26 & -181.82 & -281.63 & 0.00 & 0.00 & -39.39 & -57.91 & -37.56 & -56.74 & -452.98 & -693.55 \\
\hline$\chi_{b 2}\left(3^{3} P_{2}\right)$ & -55.75 & -67.73 & -145.60 & -182.65 & -241.15 & -319.48 & -10.04 & -11.36 & -28.45 & -33.05 & -43.01 & -52.80 & -524.01 & -667.06 \\
\hline$\chi_{b 1}\left(3^{3} P_{1}\right)$ & 0.00 & 0.00 & -214.17 & -284.75 & -216.20 & -284.87 & 0.00 & 0.00 & -35.57 & -43.03 & -44.40 & -54.40 & -510.33 & -667.05 \\
\hline$\chi_{b 0}\left(3^{3} P_{0}\right)$ & -113.71 & -160.42 & 0.00 & 0.00 & -309.51 & -418.47 & -15.72 & -19.23 & 0.00 & 0.00 & -62.82 & -79.06 & -501.76 & -677.18 \\
\hline$h_{b}\left(3^{1} P_{1}\right)$ & 0.00 & 0.00 & -226.93 & -295.84 & -205.25 & -274.16 & 0.00 & 0.00 & -41.32 & -49.28 & -39.10 & -48.42 & -512.60 & -667.70 \\
\hline$\Upsilon\left(3^{3} D_{3}\right)$ & -59.27 & -50.06 & -135.21 & -158.75 & -262.08 & -222.92 & -12.09 & -12.12 & -30.07 & -31.22 & -41.35 & -45.18 & -540.07 & -520.24 \\
\hline$\Upsilon\left(3^{3} D_{2}\right)$ & 0.00 & 0.00 & -236.94 & -229.11 & -243.69 & -285.65 & 0.00 & 0.00 & -37.77 & -39.80 & -44.57 & -48.62 & -562.97 & -603.19 \\
\hline$\Upsilon\left(3^{3} D_{1}\right)$ & -70.75 & -82.65 & -78.91 & -55.62 & -334.03 & -411.00 & -10.34 & -10.63 & -9.25 & -9.94 & -62.05 & -68.14 & -565.34 & -637.99 \\
\hline
\end{tabular}




\begin{tabular}{|c|c|c|c|c|c|c|c|c|c|c|c|c|c|c|}
\hline$\Upsilon\left(3^{1} D_{2}\right)$ & 0.00 & 0.00 & -236.85 & -249.00 & -228.98 & -241.74 & 0.00 & 0.00 & -42.63 & -44.73 & -39.90 & -43.62 & -548.36 & -579.09 \\
\hline$\Upsilon\left(3^{3} F_{4}\right)$ & -69.39 & -61.81 & -169.31 & -132.85 & -261.05 & -244.96 & -12.68 & -12.09 & -31.28 & -29.63 & -41.31 & -41.08 & -585.02 & -522.4 \\
\hline$\Upsilon\left(3^{3} F_{3}\right)$ & 0.00 & 0.00 & -273.86 & -200.06 & -241.63 & -208.98 & 0.00 & 0.00 & -37.88 & -36.94 & -44.73 & -44.64 & -598.10 & -490.62 \\
\hline$\Upsilon\left(3^{3} F_{2}\right)$ & -104.89 & 54.81 & -97.82 & -70.29 & -325.15 & -275.48 & -9.02 & -8.20 & -9.59 & -9.84 & -63.44 & -63.66 & -609.91 & -482.29 \\
\hline$\Upsilon\left(3^{1} F_{3}\right)$ & 0.00 & 0.00 & -281.25 & -208.98 & -231.33 & -206.35 & 0.00 & 0.00 & -42.46 & -41.04 & -40.96 & -40.84 & -596.00 & -497.22 \\
\hline$\Upsilon\left(3^{3} G_{5}\right)$ & -56.93 & -60.01 & -141.82 & -143.92 & -342.65 & -219.88 & -13.37 & -11.41 & -29.18 & -26.58 & -37.92 & -35.24 & -621.88 & -497.05 \\
\hline$\Upsilon\left(3^{3} G_{4}\right)$ & 0.00 & 0.00 & -310.09 & -225.76 & -272.07 & -215.82 & 0.00 & 0.00 & -37.53 & -34.67 & -41.54 & -39.36 & -661.23 & -515.61 \\
\hline$\Upsilon\left(3^{3} G_{3}\right)$ & -105.81 & -74.93 & -149.05 & -81.30 & -345.55 & -299.82 & -7.24 & -7.32 & -10.64 & -9.98 & -60.27 & -57.59 & -678.57 & -530.9 \\
\hline$\Upsilon\left(3^{1} G_{4}\right)$ & 0.00 & 0.00 & -300.09 & -233.32 & -273.56 & -204.85 & 0.00 & 0.00 & -40.70 & -37.48 & -38.49 & -36.30 & -652.84 & -511.96 \\
\hline$\Upsilon\left(4^{3} S_{1}\right)$ & -37.51 & -26.15 & -118.08 & -163.00 & -219.95 & -314.79 & -6.70 & -7.24 & -25.21 & -28.16 & -41.80 & -48.63 & -449.25 & -587.97 \\
\hline$\eta_{b}\left(4^{1} S_{0}\right)$ & 0.00 & 0.00 & -196.41 & -338.17 & -181.32 & -266.29 & 0.00 & 0.00 & -37.26 & -42.95 & -35.35 & -42.49 & -450.35 & -689.90 \\
\hline$\chi_{b 2}\left(4^{3} P_{2}\right)$ & -48.45 & -41.00 & -122.10 & -131.58 & -231.06 & -203.97 & -9.02 & -9.04 & -27.49 & -26.96 & -40.68 & -42.19 & -478.81 & -454.73 \\
\hline$\chi_{b 1}\left(4^{3} P_{1}\right)$ & 0.00 & 0.00 & -186.51 & -190.37 & -204.78 & -190.94 & 0.00 & 0.00 & -34.05 & -34.58 & -41.50 & -43.36 & -466.84 & -459.25 \\
\hline$\chi_{b 0}\left(4^{3} P_{0}\right)$ & -90.75 & -95.39 & 0.00 & 0.00 & -300.27 & -248.55 & -15.45 & -14.72 & 0.00 & 0.00 & -58.86 & -62.40 & -465.34 & -421.06 \\
\hline$h_{b}\left(4^{1} P_{1}\right)$ & 0.00 & 0.00 & -193.92 & -206.46 & -196.90 & -173.78 & 0.00 & 0.00 & -39.29 & -39.33 & -36.76 & -38.38 & -466.86 & -457.94 \\
\hline$\Upsilon\left(4^{3} D_{3}\right)$ & 51.67 & -48.85 & -153.03 & -114.14 & -235.12 & -205.93 & -10.93 & -10.26 & -28.09 & -26.27 & -37.54 & -37.05 & -526.37 & -442.50 \\
\hline$\Upsilon\left(4^{3} D_{2}\right)$ & 0.00 & 0.00 & -241.14 & -169.04 & -213.88 & -187.39 & 0.00 & 0.00 & -34.87 & -32.60 & -40.75 & -40.15 & -530.63 & -429.17 \\
\hline$\Upsilon\left(4^{3} D_{1}\right)$ & -97.12 & -53.85 & -72.32 & -50.43 & -286.05 & -256.97 & -9.60 & -8.61 & -8.37 & -7.88 & -56.78 & -56.22 & -530.24 & -433.95 \\
\hline$\Upsilon\left(4^{1} D_{2}\right)$ & 0.00 & 0.00 & -249.48 & -178.46 & -203.77 & -179.97 & 0.00 & 0.00 & -39.57 & -37.01 & -36.42 & -35.93 & -529.24 & -431.36 \\
\hline$\Upsilon\left(5^{3} S_{1}\right)$ & -35.84 & -28.67 & -117.85 & -117.03 & -195.45 & -187.51 & -6.33 & -6.02 & -23.93 & -23.54 & -39.84 & -39.50 & -419.24 & -402.28 \\
\hline$\eta_{b}\left(5^{1} S_{0}\right)$ & 0.00 & 0.00 & -172.70 & -172.07 & -167.37 & -147.05 & 0.00 & 0.00 & -35.78 & -35.31 & -33.49 & -33.88 & -409.34 & -388.31 \\
\hline$\chi_{b 2}\left(5^{3} P_{2}\right)$ & -51.88 & -38.48 & -141.94 & -101.83 & -220.37 & -179.93 & -8.73 & -8.11 & -25.26 & -23.32 & -38.13 & -36.34 & -486.31 & -388.00 \\
\hline$\chi_{b 1}\left(5^{3} P_{1}\right)$ & 0.00 & 0.00 & -215.76 & -145.97 & -194.94 & -166.57 & 0.00 & 0.00 & -32.42 & -29.47 & -38.99 & -37.48 & -482.11 & -379.49 \\
\hline$\chi_{b 0}\left(5^{3} P_{0}\right)$ & -124.51 & -70.87 & 0.00 & 0.00 & -276.93 & -248.17 & -14.71 & -13.04 & 0.00 & 0.00 & -55.43 & -53.74 & -471.57 & -385.82 \\
\hline$h_{b}\left(5^{1} P_{1}\right)$ & 0.00 & 0.00 & -224.84 & -156.57 & -185.75 & -156.94 & 0.00 & 0.00 & -37.16 & -34.08 & -34.51 & -33.10 & -482.26 & -380.69 \\
\hline$\Upsilon\left(6^{3} S_{1}\right)$ & -39.02 & -25.60 & -140.86 & -93.73 & -208.74 & -164.45 & -6.10 & -5.53 & -23.52 & -21.26 & -38.94 & -36.29 & -457.18 & -346.84 \\
\hline
\end{tabular}


\begin{tabular}{lllllllllllllll}
$\eta_{b}\left(6^{1} S_{0}\right)$ & 0.00 & 0.00 & -207.80 & -139.80 & -175.46 & -142.82 & 0.00 & 0.00 & -35.10 & -31.57 & -33.15 & -31.02 & -451.51 & -345.21 \\
\hline
\end{tabular} 
Table C.4: $1 \beta+r_{q}$ vs $3 \beta+r_{q}$ comparison for $1 S+1 S$ loop mass corrections.

\begin{tabular}{|c|c|c|c|c|c|c|c|c|c|c|c|c|c|c|}
\hline \multirow{3}{*}{$\begin{array}{l}\text { State } \\
\Upsilon \Upsilon\left(1^{3} S_{1}\right)\end{array}$} & \multicolumn{14}{|c|}{$\Omega(M)(\mathrm{MeV})$} \\
\hline & \multicolumn{2}{|c|}{$B B$} & \multicolumn{2}{|c|}{$B B^{*}$} & \multicolumn{2}{|c|}{$B^{*} B^{*}$} & \multicolumn{2}{|c|}{$B_{s} B_{s}$} & \multicolumn{2}{|c|}{$B_{s} B_{s}^{*}$} & \multicolumn{2}{|c|}{$B_{s}^{*} B_{s}^{*}$} & \multicolumn{2}{|c|}{ Total } \\
\hline & -7.67 & -2.50 & -29.63 & -10.22 & -50.10 & -18.43 & -1.56 & -0.51 & -6.04 & -2.13 & -10.24 & -3.94 & -105.26 & -37.73 \\
\hline$\eta_{b}\left(1^{1} S_{0}\right)$ & 0.00 & 0.00 & -42.38 & -13.69 & -41.01 & -14.20 & 0.00 & 0.00 & -8.69 & -2.93 & -8.43 & -3.12 & -100.51 & -33.94 \\
\hline$\Upsilon\left(2^{3} S_{1}\right)$ & -11.57 & -6.72 & -43.63 & -26.59 & -72.23 & -46.47 & -2.06 & -1.03 & -7.86 & -4.24 & -13.13 & -7.65 & -150.48 & -92.70 \\
\hline$\eta_{b}\left(2^{1} S_{0}\right)$ & 0.00 & 0.00 & -63.22 & -37.55 & -59.93 & -37.56 & 0.00 & 0.00 & -11.47 & -6.09 & -10.96 & -6.29 & -145.59 & -87.50 \\
\hline$\Upsilon\left(3^{3} S_{1}\right)$ & -13.66 & -9.71 & -49.38 & -38.32 & -79.09 & -65.83 & -2.11 & -1.17 & -7.90 & -4.78 & -13.01 & -8.63 & -165.16 & -128.44 \\
\hline$\eta_{b}\left(3^{1} S_{0}\right)$ & 0.00 & 0.00 & -71.35 & -55.90 & -65.57 & -55.00 & 0.00 & 0.00 & -11.57 & -7.12 & -10.90 & -7.35 & -159.39 & -125.37 \\
\hline$\Upsilon\left(4^{3} S_{1}\right)$ & -11.40 & 1.99 & -20.46 & -50.66 & -83.85 & -113.99 & -1.64 & -1.11 & -5.89 & -4.41 & -9.42 & -7.86 & -132.66 & -176.04 \\
\hline$\eta_{b}\left(4^{1} S_{0}\right)$ & 0.00 & 0.00 & -73.54 & -139.31 & -64.32 & -88.90 & 0.00 & 0.00 & -8.62 & -6.71 & -7.91 & -6.83 & -154.39 & -241.75 \\
\hline$\Upsilon\left(5^{3} S_{1}\right)$ & -35.84 & -5.92 & -117.85 & -28.60 & -195.45 & -33.99 & -6.33 & -0.60 & -23.93 & -3.50 & -39.84 & -2.54 & -419.24 & -75.16 \\
\hline$\eta_{b}\left(5^{1} S_{0}\right)$ & 0.00 & 0.00 & -172.70 & -37.13 & -167.37 & -17.29 & 0.00 & 0.00 & -35.78 & -4.61 & -33.49 & -2.56 & -409.34 & -61.59 \\
\hline$\Upsilon\left(6^{3} S_{1}\right)$ & -39.02 & -3.00 & -140.86 & -15.66 & -208.74 & -38.66 & -6.10 & -0.58 & -23.52 & -2.06 & -38.94 & -4.00 & -457.18 & -63.96 \\
\hline$\eta_{b}\left(6^{1} S_{0}\right)$ & 0.00 & 0.00 & -207.80 & -28.21 & -175.46 & -35.53 & 0.00 & 0.00 & -35.10 & -2.89 & -33.15 & -3.69 & -451.51 & -70.32 \\
\hline$\chi_{b 2}\left(1^{3} P_{2}\right)$ & -16.24 & -7.24 & -46.32 & -21.83 & -78.90 & -36.37 & -3.11 & -1.27 & -8.92 & -3.96 & -13.84 & -6.25 & -167.32 & -76.91 \\
\hline$\chi_{b 1}\left(1^{3} P_{1}\right)$ & 0.00 & 0.00 & -66.05 & -27.50 & -72.05 & -35.06 & 0.00 & 0.00 & -11.27 & -4.54 & -13.99 & -6.62 & -163.37 & -73.73 \\
\hline$\chi_{b 0}\left(1^{3} P_{0}\right)$ & -31.29 & -11.24 & 0.00 & 0.00 & -102.62 & -47.18 & -4.90 & -1.68 & 0.00 & 0.00 & -19.69 & -8.96 & -158.49 & -69.06 \\
\hline$h_{b}\left(1^{1} P_{1}\right)$ & 0.00 & 0.00 & -71.23 & -31.55 & -67.78 & -31.62 & 0.00 & 0.00 & -13.00 & -5.52 & -12.45 & -5.70 & -164.46 & -74.39 \\
\hline$\chi_{b 2}\left(2^{3} P_{2}\right)$ & -13.91 & -11.78 & -38.50 & -34.82 & -66.57 & -64.52 & -2.35 & -1.61 & -6.65 & -4.97 & -10.66 & -8.90 & -138.65 & -126.59 \\
\hline$\chi_{b 1}\left(2^{3} P_{1}\right)$ & 0.00 & 0.00 & -57.73 & -52.62 & -58.28 & -56.39 & 0.00 & 0.00 & -8.93 & -6.94 & -10.31 & -8.45 & -135.24 & -124.41 \\
\hline$\chi_{b 0}\left(2^{3} P_{0}\right)$ & -29.11 & -25.35 & 0.00 & 0.00 & -83.56 & -80.52 & -4.14 & -3.09 & 0.00 & 0.00 & -14.65 & -12.10 & -131.45 & -121.06 \\
\hline$h_{b}\left(2^{1} P_{1}\right)$ & 0.00 & 0.00 & -60.57 & -54.99 & -56.12 & -54.29 & 0.00 & 0.00 & -9.96 & -7.58 & -9.40 & -7.79 & -136.05 & -124.66 \\
\hline$\chi_{b 2}\left(3^{3} P_{2}\right)$ & -55.75 & -18.44 & -145.60 & -46.23 & -241.15 & -85.86 & -10.04 & -1.50 & -28.45 & -4.59 & -43.01 & -8.56 & -524.01 & -165.18 \\
\hline$\chi_{b 1}\left(3^{3} P_{1}\right)$ & 0.00 & 0.00 & -214.17 & -78.54 & -216.20 & -69.45 & 0.00 & 0.00 & -35.57 & -6.82 & -44.40 & -7.88 & -510.33 & -162.69 \\
\hline
\end{tabular}




\begin{tabular}{|c|c|c|c|c|c|c|c|c|c|c|c|c|c|c|}
\hline$\chi_{b 0}\left(3^{3} P_{0}\right)$ & -113.71 & -49.41 & 0.00 & 0.00 & -309.51 & -100.84 & -15.72 & -3.20 & 0.00 & 0.00 & -62.82 & -11.51 & -501.76 & -164.96 \\
\hline$h_{b}\left(3^{1} P_{1}\right)$ & 0.00 & 0.00 & -226.93 & -77.55 & -205.25 & -70.08 & 0.00 & 0.00 & -41.32 & -7.25 & -39.10 & -7.44 & -512.60 & -162.32 \\
\hline$\chi_{b 2}\left(4^{3} P_{2}\right)$ & -48.45 & -6.77 & -122.10 & -33.06 & -231.06 & -30.48 & -9.02 & -0.95 & -27.49 & -5.72 & -40.68 & -8.65 & -478.81 & -85.62 \\
\hline$\chi_{b 1}\left(4^{3} P_{1}\right)$ & 0.00 & 0.00 & -186.51 & -42.27 & -204.78 & -28.47 & 0.00 & 0.00 & -34.05 & -8.98 & -41.50 & -7.29 & -466.84 & -87.01 \\
\hline$\chi_{b 0}\left(4^{3} P_{0}\right)$ & -90.75 & -23.29 & 0.00 & 0.00 & -300.27 & -17.01 & -15.45 & -1.69 & 0.00 & 0.00 & -58.86 & -10.63 & -465.34 & -52.61 \\
\hline$h_{b}\left(4^{1} P_{1}\right)$ & 0.00 & 0.00 & -193.92 & -49.80 & -196.90 & -23.40 & 0.00 & 0.00 & -39.29 & -7.91 & -36.76 & -7.14 & -466.86 & -88.25 \\
\hline$\chi_{b 2}\left(5^{3} P_{2}\right)$ & -51.88 & -4.43 & -141.94 & -13.21 & -220.37 & -41.38 & -8.73 & -0.90 & -25.26 & -2.33 & -38.13 & -4.50 & -486.31 & -66.75 \\
\hline$\chi_{b 1}\left(5^{3} P_{1}\right)$ & 0.00 & 0.00 & -215.76 & -25.63 & -194.94 & -36.75 & 0.00 & 0.00 & -32.42 & -2.98 & -38.99 & -4.22 & -482.11 & -69.60 \\
\hline$\chi_{b 0}\left(5^{3} P_{0}\right)$ & -124.51 & -9.84 & 0.00 & 0.00 & -276.93 & -60.50 & -14.71 & -1.34 & 0.00 & 0.00 & -55.43 & -6.84 & -471.57 & -78.52 \\
\hline$h_{b}\left(5^{1} P_{1}\right)$ & 0.00 & 0.00 & -224.84 & -24.08 & -185.75 & -36.42 & 0.00 & 0.00 & -37.16 & -3.27 & -34.51 & -4.03 & -482.26 & -67.81 \\
\hline$\Upsilon\left(1^{3} D_{3}\right)$ & -23.46 & -11.99 & -58.60 & -31.98 & -102.94 & -55.89 & -4.22 & -1.91 & -10.66 & -5.26 & -16.04 & -8.24 & -215.92 & -115.27 \\
\hline$\Upsilon\left(1^{3} D_{2}\right)$ & 0.00 & 0.00 & -91.02 & -46.79 & -91.92 & -52.15 & 0.00 & 0.00 & -14.27 & -6.82 & -16.22 & -8.60 & -213.44 & -114.36 \\
\hline$\Upsilon\left(1^{3} D_{1}\right)$ & -29.99 & -14.17 & -27.56 & -13.29 & -123.37 & -70.00 & -4.03 & -1.77 & -3.77 & -1.72 & -22.31 & -11.84 & -211.02 & -112.78 \\
\hline$\Upsilon\left(1^{1} D_{2}\right)$ & 0.00 & 0.00 & -94.64 & -49.67 & -88.49 & -49.13 & 0.00 & 0.00 & -15.70 & -7.60 & -14.87 & -7.77 & -213.70 & -114.16 \\
\hline$\Upsilon\left(2^{3} D_{3}\right)$ & -68.78 & -15.86 & -168.12 & -41.03 & -286.50 & -80.82 & -13.25 & -1.90 & -33.37 & -5.22 & -46.45 & -9.32 & -616.48 & -154.16 \\
\hline$\Upsilon\left(2^{3} D_{2}\right)$ & 0.00 & 0.00 & -259.91 & -69.34 & -258.59 & -68.71 & 0.00 & 0.00 & -41.97 & -7.69 & -49.80 & -8.90 & -610.27 & -154.63 \\
\hline$\Upsilon\left(2^{3} D_{1}\right)$ & -89.04 & -24.70 & -78.12 & -22.22 & -347.79 & -91.24 & -11.16 & -2.27 & -10.31 & -2.22 & -69.24 & -12.17 & -605.65 & -154.82 \\
\hline$\Upsilon\left(2^{1} D_{2}\right)$ & 0.00 & 0.00 & -270.66 & -69.99 & -247.71 & -67.44 & 0.00 & 0.00 & -47.35 & -8.16 & -44.67 & -8.35 & -610.38 & -153.95 \\
\hline$\Upsilon\left(3^{3} D_{3}\right)$ & -59.27 & -3.71 & -135.21 & -39.99 & -262.08 & -32.18 & -12.09 & -1.75 & -30.07 & -4.60 & -41.35 & -8.55 & -540.07 & -90.79 \\
\hline$\Upsilon\left(3^{3} D_{2}\right)$ & 0.00 & 0.00 & -236.94 & -46.44 & -243.69 & -90.53 & 0.00 & 0.00 & -37.77 & -7.27 & -44.57 & -7.82 & -562.97 & -152.06 \\
\hline$\Upsilon\left(3^{3} D_{1}\right)$ & -70.75 & -20.89 & -78.91 & -3.35 & -334.03 & -138.37 & -10.34 & -2.55 & -9.25 & -2.22 & -62.05 & -10.62 & -565.34 & -178.00 \\
\hline$\Upsilon\left(3^{1} D_{2}\right)$ & 0.00 & 0.00 & -236.85 & -57.24 & -228.98 & -63.87 & 0.00 & 0.00 & -42.63 & -7.52 & -39.90 & -7.47 & -548.36 & -136.10 \\
\hline$\Upsilon\left(4^{3} D_{3}\right)$ & -57.91 & -5.89 & -144.95 & -12.32 & -218.73 & -48.77 & -9.65 & -1.23 & -25.28 & -2.62 & -37.15 & -5.73 & -493.66 & -76.56 \\
\hline$\Upsilon\left(4^{3} D_{2}\right)$ & -2.06 & 0.00 & -220.71 & -26.02 & -198.84 & -37.65 & -0.26 & 0.00 & -33.44 & -3.37 & -37.33 & -4.69 & -492.65 & -71.73 \\
\hline$\Upsilon\left(4^{3} D_{1}\right)$ & -86.66 & -5.71 & -70.50 & -10.79 & -261.02 & -53.30 & -9.98 & -0.91 & -9.50 & -1.00 & -50.69 & -6.62 & -488.35 & -78.32 \\
\hline$\Upsilon\left(4^{1} D_{2}\right)$ & 0.00 & 0.00 & -232.83 & -24.39 & -188.80 & -39.30 & 0.00 & 0.00 & -37.23 & -3.69 & -34.26 & -4.75 & -493.12 & -72.13 \\
\hline
\end{tabular}




\begin{tabular}{|c|c|c|c|c|c|c|c|c|c|c|c|c|c|c|}
\hline$\Upsilon\left(1^{3} F_{4}\right)$ & -22.36 & -15.98 & -51.33 & -39.86 & -94.55 & -75.10 & -3.73 & -2.31 & -8.75 & -5.98 & -13.09 & -9.68 & -193.81 & -148.91 \\
\hline$\Upsilon\left(1^{3} F_{3}\right)$ & 0.00 & 0.00 & -86.25 & -64.67 & -81.67 & -67.09 & 0.00 & 0.00 & -12.24 & -8.39 & -13.09 & -9.79 & -193.25 & -149.94 \\
\hline$\Upsilon\left(1^{3} F_{2}\right)$ & -27.61 & -19.12 & -32.43 & -23.44 & -107.82 & -89.51 & -3.14 & -2.05 & -3.83 & -2.62 & -18.16 & -13.65 & -192.98 & -150.39 \\
\hline$\Upsilon\left(1^{1} F_{3}\right)$ & 0.00 & 0.00 & -87.65 & -66.33 & -80.00 & -64.90 & 0.00 & 0.00 & -13.10 & -8.97 & -12.25 & -9.13 & -192.99 & -149.32 \\
\hline$\Upsilon\left(2^{3} F_{4}\right)$ & -32.69 & -22.81 & -60.10 & -49.57 & -112.80 & -110.33 & -3.45 & -2.04 & -7.87 & -5.25 & -11.83 & -9.46 & -228.76 & -199.47 \\
\hline$\Upsilon\left(2^{3} F_{3}\right)$ & 0.00 & 0.00 & -137.90 & -116.38 & -88.98 & -85.56 & 0.00 & 0.00 & -11.31 & -8.08 & -11.63 & -8.95 & -249.82 & -218.98 \\
\hline$\Upsilon\left(2^{3} F_{2}\right)$ & -28.86 & -40.95 & -60.81 & -51.84 & -113.13 & -108.28 & -3.16 & -2.22 & -3.65 & -2.81 & -16.04 & -12.25 & -225.66 & -218.35 \\
\hline$\Upsilon\left(2^{1} F_{3}\right)$ & 0.00 & 0.00 & -130.11 & -109.43 & -89.58 & -86.73 & 0.00 & 0.00 & -12.01 & -8.42 & -10.94 & -8.53 & -242.64 & -213.11 \\
\hline$\Upsilon\left(3^{3} F_{4}\right)$ & -20.21 & -9.87 & -49.98 & -13.14 & -47.65 & -54.70 & -2.54 & -1.76 & -8.79 & -5.36 & -14.95 & -11.69 & -144.12 & -96.52 \\
\hline$\Upsilon\left(3^{3} F_{3}\right)$ & 0.00 & 0.00 & -60.68 & -26.43 & -50.70 & -29.62 & 0.00 & 0.00 & -8.75 & -5.96 & -12.96 & -9.22 & -133.10 & -71.23 \\
\hline$\Upsilon\left(3^{3} F_{2}\right)$ & -24.34 & -3.58 & -14.82 & -13.45 & -69.16 & -31.75 & -2.75 & -0.65 & -1.39 & -1.49 & -16.89 & -11.68 & -129.35 & -62.60 \\
\hline$\Upsilon\left(3^{1} F_{3}\right)$ & 0.00 & 0.00 & -67.75 & -25.20 & -46.72 & -35.18 & 0.00 & 0.00 & -10.53 & -6.87 & -12.77 & -9.35 & -137.77 & -76.59 \\
\hline$\Upsilon\left(1^{3} G_{5}\right)$ & -27.40 & -19.18 & -58.43 & -45.61 & -115.89 & -94.53 & -4.11 & -2.49 & -9.13 & -6.18 & -13.82 & -10.52 & -228.78 & -178.50 \\
\hline$\Upsilon\left(1^{3} G_{4}\right)$ & 0.00 & 0.00 & -109.30 & -82.23 & -95.34 & -80.25 & 0.00 & 0.00 & -13.35 & -9.25 & -13.56 & -10.30 & -231.55 & -182.03 \\
\hline$\Upsilon\left(1^{3} G_{3}\right)$ & -39.47 & -26.11 & -47.26 & -34.41 & -122.81 & -104.99 & -3.42 & -2.27 & -4.55 & -3.23 & -18.88 & -14.36 & -236.38 & -185.36 \\
\hline$\Upsilon\left(1^{1} G_{4}\right)$ & 0.00 & 0.00 & -108.73 & -82.42 & -94.93 & -79.15 & 0.00 & 0.00 & -14.02 & -9.66 & -12.89 & -9.79 & -230.56 & -181.03 \\
\hline$\Upsilon\left(2^{3} G_{5}\right)$ & -14.54 & -20.77 & -56.83 & -59.68 & -63.04 & -91.98 & -4.15 & -2.16 & -8.52 & -5.20 & -13.21 & -9.76 & -160.29 & -189.55 \\
\hline$\Upsilon\left(2^{3} G_{4}\right)$ & 0.00 & 0.00 & -70.65 & -51.39 & -88.95 & -95.18 & 0.00 & 0.00 & -13.36 & -8.71 & -12.47 & -8.95 & -185.43 & -164.23 \\
\hline$\Upsilon\left(2^{3} G_{3}\right)$ & -31.60 & 1.65 & -17.45 & -2.96 & -138.46 & -134.08 & -5.13 & -2.96 & -4.91 & -3.43 & -17.09 & -12.16 & -214.65 & -153.95 \\
\hline$\Upsilon\left(2^{1} G_{4}\right)$ & 0.00 & 0.00 & -77.49 & -63.10 & -78.48 & -90.07 & 0.00 & 0.00 & -13.79 & -8.87 & -11.97 & -8.66 & -181.73 & -170.70 \\
\hline$\Upsilon\left(3^{3} G_{5}\right)$ & -19.87 & -13.10 & -61.29 & -33.48 & -150.81 & -30.64 & -4.40 & -1.07 & -9.82 & -3.02 & -16.64 & -5.34 & -262.82 & -86.66 \\
\hline$\Upsilon\left(3^{3} G_{4}\right)$ & -5.72 & 0.00 & -131.20 & -37.95 & -123.45 & -38.68 & -0.69 & 0.00 & -13.12 & -5.26 & -16.31 & -6.22 & -290.50 & -88.11 \\
\hline$\Upsilon\left(3^{3} G_{3}\right)$ & -48.46 & -10.50 & -79.36 & -7.75 & -146.78 & -56.86 & -2.28 & -1.40 & -6.14 & -2.10 & -21.23 & -9.36 & -304.24 & -87.97 \\
\hline$\Upsilon\left(3^{1} G_{4}\right)$ & 0.00 & 0.00 & -134.24 & -42.53 & -120.15 & -34.71 & 0.00 & 0.00 & -14.92 & -5.33 & -15.16 & -5.67 & -284.48 & -88.24 \\
\hline$\Upsilon\left(1^{3} H_{6}\right)$ & -98.85 & -21.32 & -196.08 & -48.64 & -439.15 & -111.19 & -14.51 & -2.55 & -31.18 & -6.18 & -45.12 & -10.63 & -824.90 & -200.51 \\
\hline$\Upsilon\left(1^{3} H_{5}\right)$ & 0.00 & 0.00 & -443.26 & -100.69 & -337.90 & -90.12 & 0.00 & 0.00 & -45.31 & -9.51 & -45.33 & -10.34 & -871.80 & -210.66 \\
\hline
\end{tabular}


\begin{tabular}{lllllllllllllll}
$\Upsilon\left(1^{3} H_{4}\right)$ & -167.81 & -35.89 & -226.31 & -48.59 & -417.76 & -115.00 & -11.26 & -2.35 & -15.47 & -3.49 & -64.34 & -14.48 & -902.95 & -219.79 \\
\hline
\end{tabular} 


\section{Bibliography}

[1] M. Gell-Mann. "A schematic model of baryons and mesons". In: Physics Letters 8 (1964), pp. 214 -215. ISSN: 0031-9163. DOI: http://dx.doi.org/10.1016/ S0031-9163(64)92001-3.

[2] G. Zweig. "An SU(3) model for strong interaction symmetry and its breaking. Version 1". In: CERN Report CERN-TH-401 (1964).

[3] Andre Martin. "A Simultaneous Fit of B anti-B, C anti-C, S anti-S, (BCS Pairs) and C anti-S Spectra". In: Phys. Lett. B100 (1981), pp. 511-514. DoI: $10.1016 / 0370-2693(81) 90617-1$.

[4] R. A. Bertlmann and Andre Martin. "Inequalities on Heavy Quark - Anti-Quark Systems". In: Nucl. Phys. B168 (1980), pp. 111-136. DOI: 10 . 1016/0550$3213(80) 90279-5$.

[5] E Eichten et al. "Charmonium: the model". In: Physical Review D 17 (1978), p. 3090 .

[6] Wilfried Buchmüller and S-HH Tye. "Quarkonia and Quantum Chromodynamics". In: Physical Review D 24 (1981), p. 132.

[7] Stephen Godfrey and Nathan Isgur. "Mesons in a relativized quark model with chromodynamics". In: Physical Review D 32 (1985), p. 189. 
[8] Hua-Xing Chen et al. "A review of the open charm and open bottom systems". In: Reports on Progress in Physics 80 (2017), p. 076201.

[9] Eric S. Swanson. "The New heavy mesons: A Status report". In: Phys. Rept. 429 (2006), pp. 243-305. DOI: 10 . $1016 /$ j . physrep . 2006 . 04 . 003. arXiv: hep-ph/0601110 [hep-ph].

[10] B. Aubert et al. "Observation of a Narrow Meson State Decaying to $D_{s}^{+} \pi^{0}$ at a Mass of $2.32 \mathrm{GeV} / \mathrm{c}^{2}$ ". In: Phys. Rev. Lett. 90 (2003), p. 242001. DoI: 10.1103/PhysRevLett. 90.242001 .

[11] S.-K. Choi et al. "Observation of a Narrow Charmoniumlike State in Exclusive $B^{ \pm} \rightarrow K^{ \pm} \pi^{+} \pi^{-} J / \psi$ Decays". In: Phys. Rev. Lett. 91 (2003), p. 262001. DoI: 10.1103/PhysRevLett.91.262001.

[12] C. Patrignani et al. "Review of Particle Physics". In: Chin. Phys. C40 (2016), p. 100001. DOI: $10.1088 / 1674-1137 / 40 / 10 / 100001$.

[13] Mark Thomson. Modern particle physics. Cambridge University Press, 2013. ISBN: 9781107034266.

[14] O. W. Greenberg. "Spin and Unitary Spin Independence in a Paraquark Model of Baryons and Mesons". In: Phys. Rev. Lett. 13 (1964), pp. 598-602. DoI: 10.1103/PhysRevLett.13.598.

[15] Harald Fritzsch, Murray Gell-Mann, and Heinrich Leutwyler. "Advantages of the color octet gluon picture". In: Physics Letters B 47 (1973), pp. 365-368.

[16] A. De Rújula, Howard Georgi, and S. L. Glashow. "Hadron masses in a gauge theory". In: Phys. Rev. D 12 (1975), pp. 147-162. DOI: 10.1103/PhysRevD. 12.147. 
[17] RJ Jaffe. "Multiquark hadrons. I. Phenomenology of $Q^{2} \bar{Q}^{2}$ mesons". In: Physical Review D 15 (1977), p. 267.

[18] Robert L Jaffe. "Multiquark hadrons. II. methods". In: Physical Review D 15 (1977), p. 281.

[19] John Weinstein and Nathan Isgur. "Do Multiquark Hadrons Exist?" In: Phys. Rev. Lett. 48 (1982), pp. 659-662. DOI: 10.1103/PhysRevLett.48.659.

[20] R. Aaij et al. "Observation of $J / \psi p$ Resonances Consistent with Pentaquark States in $\Lambda_{b}^{0} \rightarrow J / \psi K^{-} p$ Decays". In: Phys. Rev. Lett. 115 (2015), p. 072001. DOI: $10.1103 /$ PhysRevLett.115.072001.

[21] D. Griffiths. Introduction to Elementary Particles. Wiley, 2008. ISBN: 9783527406012 .

[22] Stephen Gasiorowicz. Quantum physics. John Wiley \& Sons, 2007.

[23] B Numerov. "Note on the numerical integration of $\frac{d^{2} x}{d t^{2}}=f(x, t)$ ". In: $A s$ tronomische Nachrichten 230 (1927), pp. 359-364.

[24] T. Barnes and E. S. Swanson. "Hadron loops: General theorems and application to charmonium". In: Phys. Rev. C 77 (2008), p. 055206. DOI: 10.1103/ PhysRevC.77.055206.

[25] Nils A. Tornqvist. "The Meson Mass Spectrum and Unitarity". In: Annals Phys. 123 (1979), p. 1. DOI: 10.1016/0003-4916(79)90262-8.

[26] K. Heikkila, Seiji Ono, and N. A. Tornqvist. "Heavy $c \bar{c}$ and $b \bar{b}$ quarkonium states and unitarity effects". In: Phys. Rev. D29 (1984). [Erratum: Phys. Rev.D29,2136(1984)], p. 110. DOI: 10 . 1103/PhysRevD . 29 .2136 , 10 . 1103 / PhysRevD . 29.110. 
[27] S. Ono and N. A. Tornqvist. "Continuum Mixing and Coupled Channel Effects in $c \bar{c}$ and $b \bar{b}$ Quarkonium". In: Z. Phys. C23 (1984), p. 59. DOI: $10.1007 /$ BF01558041.

[28] E Van Beveren, C Dullemond, and G Rupp. "Spectra and strong decays of $c \bar{c}$ and $b \bar{b}$ states". In: Physical Review D 21 (1980), p. 772.

[29] Pedro J. de A. Bicudo and José E. F. T. Ribeiro. "Generalized resonating-groupmethod equations in a ${ }^{3} P_{0} q \bar{q}$ condensed vacuum. The $\varphi$ and $\rho$ resonances". In: Phys. Rev. D 42 (1990), pp. 1635-1650. DoI: 10.1103/PhysRevD.42.1635.

[30] T. Barnes, S. Godfrey, and E. S. Swanson. "Higher charmonia". In: Phys. Rev. D 72 (2005), p. 054026. DOI: 10.1103/PhysRevD.72.054026.

[31] Dae Sung Hwang and Do-Won Kim. "Mass of $D_{s J}^{*}(2317)$ and coupled channel effect". In: Physics Letters B 601 (2004), pp. 137-143.

[32] Yu S Kalashnikova. "Coupled-channel model for charmonium levels and an option for X (3872)". In: Physical Review D 72 (2005), p. 034010.

[33] T. J. Burns. "Angular momentum coefficients for meson strong decay and unquenched quark models". In: Phys. Rev. D 90 (2014), p. 034009. DoI: 10.1103/ PhysRevD.90.034009.

[34] Eric W. Weisstein. Sokhotsky's Formula. MathWorld. 2017. URL: http : // mathworld.wolfram.com/SokhotskysFormula.html.

[35] Eric W. Weisstein. Cauchy Principal Value. MathWorld. 2017. URL: http:// mathworld.wolfram.com/CauchyPrincipalValue.html.

[36] E. S. Ackleh, Ted Barnes, and E. S. Swanson. "On the mechanism of open flavor strong decays". In: Phys. Rev. D54 (1996), pp. 6811-6829. DoI: 10.1103/ PhysRevD.54.6811. arXiv: hep-ph/9604355 [hep-ph]. 
[37] S Okubo, G Zweig, and J Iizuka. "Symmetries in elementary particle physics". In: Phys. Lett 5 (1963), p. 165.

[38] G Zweig. "CERN Report No. 8419/TH412 (1964); I. Iizuka”. In: Prog. Theor. Phys. Suppl 37 (1966), p. 21.

[39] L Micu. "Decay rates of meson resonances in a quark model". In: Nuclear Physics B 10 (1969), pp. 521-526.

[40] A. L. Yaouanc et al. " "Naive" Quark-Pair-Creation Model of Strong-Interaction Vertices". In: Phys. Rev. D 8 (1973), pp. 2223-2234. DOI: 10.1103/PhysRevD. 8. 2223.

[41] A.L. Yaouanc. Hadron Transitions in the Quark Model. Gordon and Breach Science Publishers, 1988. ISBN: 9782881242144.

[42] Hideki Yukawa. "On the interaction of elementary particles. I". In: Nippon Sugaku-Buturigakkwai Kizi Dai 3 Ki 17 (1935), pp. 48-57.

[43] J.J.J. Kokkedee. The quark model in particle physics: lectures given at CERN during the fall of 196\%. Instituut voor Theoretische Fysica, Universiteit van Nijmegen, 1967.

[44] A. Le Yaouanc et al. "Resonant Partial Wave Amplitudes in $\pi+n \rightarrow \pi+\pi+n$ According to the Naive Quark Pair Creation Model". In: Phys. Rev. D11 (1975), p. 1272. DOI: 10.1103/PhysRevD.11.1272.

[45] Stephen Godfrey and Kenneth Moats. "Bottomonium mesons and strategies for their observation". In: Physical Review D 92 (2015), p. 054034.

[46] G. Blundell. "Meson properties in the quark model: A look at some outstanding problems". PhD thesis. Carleton U., 1996. arXiv: hep-ph/9608473 [hep-ph]. 
[47] Yu Lu, Muhammad Naeem Anwar, and Bing-Song Zou. "Coupled-channel effects for the bottomonium with realistic wave functions". In: Phys. Rev. D 94 (2016), p. 034021. DOI: 10.1103/PhysRevD.94.034021.

[48] M. Brack and R. K. Bhaduri. "Baryon self energies due to pion - quark coupling". In: Phys. Rev. D35 (1987), pp. 3451-3461. DOI: 10.1103/PhysRevD . 35.3451.

[49] J. Ferretti and E. Santopinto. "Higher mass bottomonia". In: Phys. Rev. D90 (2014), p. 094022. DOI: 10.1103/PhysRevD . 90 .094022. arXiv: 1306 . 2874 $[$ hep-ph].

[50] J. Ferretti, G. Galat, and E. Santopinto. "Interpretation of the X(3872) as a charmonium state plus an extra component due to the coupling to the mesonmeson continuum". In: Phys. Rev. C88 (2013), p. 015207. DOI: 10 . 1103 / PhysRevC.88.015207. arXiv: 1302.6857 [hep-ph].

[51] Paul Geiger and Nathan Isgur. "The Quenched Approximation in the Quark Model". In: Phys. Rev. D41 (1990), p. 1595. DOI: 10.1103/PhysRevD .41.1595.

[52] Paul Geiger and Nathan Isgur. "Reconciling the Okubo-Zweig-Iizuka rule with strong pair creation". In: Phys. Rev. D 44 (1991), pp. 799-808. DoI: 10.1103/ PhysRevD . 44.799.

[53] M. Jacob and G. C. Wick. "On the general theory of collisions for particles with spin". In: Annals Phys. 7 (1959). [Reprinted in Annals Phys.281,774(2000)], pp. 404-428. DOI: $10.1016 / 0003-4916(59) 90051-X$.

[54] Jeffrey D. Richman. "An Experimenter's Guide to the Helicity Formalism”. In: Report CALT-68-1148 (1984).

[55] Eric W. Weisstein. Associated Laguerre Polynomial. MathWorld. 2017. URL: http://mathworld.wolfram.com/AssociatedLaguerrePolynomial.html. 
[56] W Roberts and B Silvestre-Brac. "General method of calculation of any hadronic decay in the ${ }^{3} P_{0}$ model". In: Few-Body Systems 11 (1992), pp. 171193.

[57] Wolfram Research, Inc. Mathematica, Version 11.1. Champaign, IL, 2017.

[58] C. Michael. "Long-Range Spin-Orbit Potential". In: Phys. Rev. Lett. 56 (1986), pp. 1219-1221. DOI: 10.1103/PhysRevLett.56.1219.

[59] T. Barnes. "Strong decays: Past, present and future". In: AIP Conf. Proc. 717 (2004), pp. 625-635. DOI: 10.1063/1.1799772. arXiv: hep-ph/0311102 $[$ hep-ph].

[60] E. Eichten et al. "Charmonium: Comparison with Experiment". In: Phys. Rev. D21 (1980), p. 203. DOI: 10.1103/PhysRevD.21.203.

[61] J. Ferretti et al. "Bottomonium self-energies due to the coupling to the mesonmeson continuum". In: Phys. Rev. C86 (2012), p. 015204. DOI: 10 . 1103 / PhysRevC.86.015204.

[62] Jia-Feng Liu and Gui-Jun Ding. "Bottomonium spectrum with coupled-channel effects". In: The European Physical Journal C 72 (2012), p. 1981. ISSN: 14346052. DOI: $10.1140 / \mathrm{epjc} / \mathrm{s} 10052-012-1981-6$.

[63] Pablo G. Ortega et al. "Molecular components in $P$-wave charmed-strange mesons". In: Phys. Rev. D 94 (2016), p. 074037. DOI: 10 . 1103 / PhysRevD . 94.074037.

[64] T. Barnes et al. "Higher quarkonia". In: Phys. Rev. D 55 (1997), pp. 4157-4188. DOI: $10.1103 /$ PhysRevD.55.4157. 
[65] Stephen Godfrey and Kenneth Moats. "Properties of excited charm and charmstrange mesons". In: Phys. Rev. D 93 (2016), p. 034035. DOI: 10 . 1103 / PhysRevD.93.034035.

[66] S Capstick. "Baryon Spectroscopy in the Quark Model". In: Baryons 2002. 2003, pp. 17-28.

[67] Yu Lu, Muhammad Naeem Anwar, and Bing-Song Zou. "How Large is the Contribution of Excited Mesons in Coupled-Channel Effects?" In: Phys. Rev. D95 (2017), p. 034018. DOI: 10.1103/PhysRevD.95.034018. arXiv: 1701.00692 [hep-ph].

[68] E Hiyama, Y Kino, and M Kamimura. "Gaussian expansion method for fewbody systems". In: Progress in Particle and Nuclear Physics 51 (2003), pp. 223307.

[69] D. et. al. Besson. "Observation of a narrow resonance of mass $2.46 \mathrm{GeV} / c^{2}$ decaying to $D_{s}^{*+} \pi^{0}$ and confirmation of the $D_{\mathrm{sJ}}^{*}(2317)$ state". In: Phys. Rev. D 68 (2003), p. 032002. DOI: 10.1103/PhysRevD.68.032002.

[70] J. M. Flynn and Nathan Isgur. "Heavy quark symmetry: Ideas and applications". In: J. Phys. G18 (1992), pp. 1627-1644. DOI: 10.1088/0954-3899/18/ 10/004. arXiv: hep-ph/9207223 [hep-ph].

[71] Stephen Godfrey and Richard Kokoski. "Properties of $P$-wave mesons with one heavy quark". In: Phys. Rev. D 43 (1991), pp. 1679-1687. DOI: 10 . 1103 / PhysRevD .43.1679.

[72] D. Ebert, R. N. Faustov, and V. O. Galkin. "Heavy-light meson spectroscopy and Regge trajectories in the relativistic quark model". In: The European Physical Journal C 66 (2010), pp. 197-206. ISSN: 1434-6052. DOI: 10.1140/epjc/ s10052-010-1233-6. 
[73] R. Aaij et al. "Observation of Overlapping Spin-1 and Spin-3 $\bar{D}^{0} K^{-}$Resonances at Mass $2.86 \mathrm{GeV} / c^{2 "}$. In: Phys. Rev. Lett. 113 (2014), p. 162001. DoI: 10. 1103/PhysRevLett.113.162001.

[74] R. Aaij et al. "Dalitz plot analysis of $B_{s}^{0} \rightarrow \bar{D}^{0}+K^{-}+\pi^{+}$decays". In: Phys. Rev. D 90 (2014), p. 072003. DOI: 10.1103/PhysRevD.90.072003.

[75] Thomas E Browder, Sandip Pakvasa, and Alexey A Petrov. "Comment on the new $D_{s}+\pi^{0}$ resonances". In: Physics Letters $B 578$ (2004), pp. 365 -368. ISSN: 0370-2693. DOI: http://dx.doi.org/10.1016/j.physletb.2003.10.067.

[76] P. Colangelo, F. De Fazio, and R. Ferrandes. "Excited charmed mesons: Observations, analyses and puzzles". In: Mod. Phys. Lett. A19 (2004), pp. 2083-2102. DOI: 10.1142/S0217732304015269. arXiv: hep-ph/0407137 [hep-ph].

[77] Eef van Beveren and George Rupp. "Observed $D_{s}(2317)$ and Tentative $D(2100-$ 2300) as the Charmed Cousins of the Light Scalar Nonet". In: Phys. Rev. Lett. 91 (2003), p. 012003. DOI: 10.1103/PhysRevLett.91.012003.

[78] Eef van Beveren and George Rupp. "Continuum bound states $K_{L}, D_{1}(2420)$, $D_{s l}(2536)$ and their partners $K_{S}, D_{1}(2400), D_{s J}^{*}(2463)$ ". In: Eur. Phys. J. C32 (2004), pp. 493-499. DOI: 10 .1140/epjc/s2003-01465-0. arXiv: hep-ph/ 0306051 [hep-ph].

[79] Yu. A. Simonov and J. A. Tjon. "Coupled-channel analysis of the $D$ and $D_{s}$ mesons". In: Phys. Rev. D 70 (2004), p. 114013. DOI: 10.1103/PhysRevD.70. 114013.

[80] Feng-Kun Guo, Siegfried Krewald, and Ulf-G. Meissner. "Hadronic-loop induced mass shifts in scalar heavy-light mesons". In: Phys. Lett. B665 (2008), pp. 157163. DOI: 10.1016/j.physletb.2008.06.008. arXiv: 0712.2953 [hep-ph]. 
[81] Ian Woo Lee et al. "Chiral radiative corrections and $D_{s}(2317) / D(2308)$ mass puzzle". In: Eur. Phys. J. C49 (2007), pp. 737-741. DOI: 10 . 1140 / epjc / s10052-006-0149-7. arXiv: hep-ph/0412210 [hep-ph].

[82] Zhi-Yong Zhou and Zhiguang Xiao. "Hadron loops effect on mass shifts of the charmed and charmed-strange spectra". In: Phys. Rev. D84 (2011), p. 034023. DOI: 10.1103/PhysRevD.84.034023. arXiv: 1105.6025 [hep-ph] .

[83] M. R. Pennington and D. J. Wilson. "Decay channels and charmonium massshifts". In: Phys. Rev. D76 (2007), p. 077502. DOI: 10.1103/PhysRevD . 76. 077502. arXiv: 0704.3384 [hep-ph].

[84] J. Segovia, D. R. Entem, and F. Fernández. "Scaling of the ${ }^{3} P_{0}$ Strength in Heavy Meson Strong Decays". In: Phys. Lett. B715 (2012), pp. 322-327. DoI: 10.1016/j.physletb.2012.08.005. arXiv: 1205.2215 [hep-ph].

[85] John Kogut and Leonard Susskind. "Hamiltonian formulation of Wilson's lattice gauge theories". In: Phys. Rev. D 11 (1975), pp. 395-408. DoI: 10.1103 / PhysRevD.11.395.

[86] Nathan Isgur and Jack Paton. "A flux tube model for hadrons". In: Physics Letters B 124 (1983), pp. 247-251.

[87] Richard Kokoski and Nathan Isgur. "Meson decays by flux-tube breaking". In: Phys. Rev. D 35 (1987), pp. 907-933. DOI: 10.1103/PhysRevD.35.907.

[88] A. Messiah. Quantum Mechanics. Dover books on physics. Dover Publications, 1961. ISBN: 9780486409245. 